\title{
Structural basis for enzymatic photocatalysis in chlorophyll biosynthesis
}

DOI:

10.1038/s41586-019-1685-2

Document Version

Accepted author manuscript

Link to publication record in Manchester Research Explorer

\section{Citation for published version (APA):}

Zhang, S., Heyes, D. J., Feng, L., Sun, W., Johannissen, L. O., Liu, H., Levy, C. W., Li, X., Yang, J., Yu, X., Lin, M., Hardman, S. J. O., Hoeven, R., Sakuma, M., Hay, S., Leys, D., Rao, Z., Zhou, A., Cheng, Q., \& Scrutton, N. S. (2019). Structural basis for enzymatic photocatalysis in chlorophyll biosynthesis. Nature, 574, 722-725. https://doi.org/10.1038/s41586-019-1685-2

Published in:

Nature

\section{Citing this paper}

Please note that where the full-text provided on Manchester Research Explorer is the Author Accepted Manuscript or Proof version this may differ from the final Published version. If citing, it is advised that you check and use the publisher's definitive version.

\section{General rights}

Copyright and moral rights for the publications made accessible in the Research Explorer are retained by the authors and/or other copyright owners and it is a condition of accessing publications that users recognise and abide by the legal requirements associated with these rights.

\section{Takedown policy}

If you believe that this document breaches copyright please refer to the University of Manchester's Takedown Procedures [http://man.ac.uk/04Y6Bo] or contact uml.scholarlycommunications@manchester.ac.uk providing relevant details, so we can investigate your claim.

\section{OPEN ACCESS}




\section{Structural basis for enzymatic photocatalysis in chlorophyll}

\section{biosynthesis}

Shaowei Zhang*1 ${ }^{1}$ Derren J. Heyes*1, Lingling Feng*3 ${ }^{*}$, Wenli Sun*2, Linus O.

Johannissen* ${ }^{1}$, Huanting Liu ${ }^{6}$, Colin W. Levy ${ }^{1}$, Xuemei $\mathrm{Li}^{4}$, Ji Yang ${ }^{6}$, Xiaolan $\mathrm{Yu}^{6}$, Min Lin ${ }^{2}$, Samantha J. O. Hardman ${ }^{1}$, Robin Hoeven ${ }^{1}$, Michiyo Sakuma ${ }^{1}$, Sam Hay ${ }^{1}$, David Leys ${ }^{1}$, Zihe Rao ${ }^{4}$, Aiwu Zhou ${ }^{\star 3}$, Qi Cheng ${ }^{\$ 2,5,6}$ and Nigel S. Scrutton ${ }^{\$ 1}$

${ }^{1}$ Manchester Institute of Biotechnology, The University of Manchester, 131 Princess Street, Manchester M1 7DN, UK

${ }^{2}$ Biotechnology Research Institute, Chinese Academy of Agricultural Sciences, Beijing 100081, China

${ }^{3}$ Department of Pathophysiology, Key Laboratory of Cell Differentiation and Apoptosis of the Chinese Ministry of Education, Shanghai Jiao Tong University School of Medicine, 280 South Chongqing Rd., Shanghai 200025, China

${ }^{4}$ National Laboratory of Biomacromolecules, Institute of Biophysics, Chinese Academy of Sciences, Beijing 100101, China

${ }^{5}$ College of Biotechnology and Bioengineering, Zhejiang University of Technology, Hangzhou 310014, China ${ }^{6}$ C4-101, Nitrogen Fixation Laboratory, Qi Institute, 555 Chuangye Road, Dayun Town, Jiashan County, Jiaxing 314000, Zhejiang Province, China

*These authors contributed equally to this work.

Correspondence can be addressed to: nigel.scrutton@manchester.ac.uk; chengqi@caas.cn; awz20@shsmu.edu.cn 
The enzyme protochlorophyllide oxidoreductase (POR) catalyses a lightdependent step in chlorophyll biosynthesis that is essential to photosynthesis and ultimately all life on Earth. ${ }^{1-3}$ POR, which is one of three known light-dependent enzymes, ${ }^{4,5}$ catalyzes reduction of the photosensitizer and substrate protochlorophyllide to form the pigment chlorophyllide. Despite its biological importance, a structural basis for POR photocatalysis has remained elusive. Here, we report crystal structures of cyanobacterial PORs from Thermosynechococcus elongatus and Synechocystis sp. in their free forms, and in complex with nicotinamide coenzyme. Our structural models and simulations of the ternary protochlorophyllide-NADPH-POR complex have identified multiple interactions in the POR active site that are important for protochlorophyllide binding, photosensitization and photochemical conversion to chlorophyllide. We demonstrate the importance of active-site architecture and protochlorophyllide structure in experiments using POR variants and protochlorophyllide analogues. These studies reveal how the POR active site facilitates light-driven reduction of protochlorophyllide by localized hydride transfer from NADPH and long-range proton transfer along structurally defined proton-transfer pathways.

As the light-driven step in the chlorophyll biosynthetic pathway (Fig. 1), the POR reaction acts as the trigger for the germination of seedlings =in plants and provokes a marked change in the morphological development of the plant. ${ }^{2,3}$ Given this crucial biological role, POR has been the focus of numerous mechanistic and biophysical investigations. A combination of time-resolved (at the femtosecond-to-second scale) and cryogenic spectroscopy methods have provided some understanding of the mechanism of POR photocatalysis in a range of photosynthetic organisms, including cyanobacteria and plants. Picosecond excited-state dynamics in the protochlorophyllide (Pchlide) molecule are thought to result in excited state interactions between the substrate and active-site residues that are necessary to trigger the subsequent reaction chemistry. ${ }^{6-12}$ This involves sequential transfer of a hydride equivalent from NADPH and a proton transfer from either an active site residue or solvent. Proton transfer is reliant on solvent dynamics and an implied network of extended protein motions that occur on the microsecond timescale. ${ }^{13-17}$ Hydride transfer from NADPH is not concerted, but occurs in a stepwise manner that involves 
Pchlide excited-state electron transfer from NADPH followed by a proton-coupled electron transfer, ${ }^{18}$ which represents the first example of the stepwise transfer of a hydride equivalent reported in biology. These time-resolved studies have provided insight into the chemistry of catalysis across a wide range of timescales (from the femtosecond to the second), but the required structural basis of POR photocatalysis has remained unknown. This structural context is urgently required to understand how substrate binding, excited-state chemistry, bond making and/or breaking and the dynamics of photocatalysis are controlled by protein structure.

Here we report the crystal structures of the apo-POR enzyme from T.elongatus(RCSB Protein Data Bank (PDB) code 6RNV), and NADPH-bound POR from both Synechocystis sp, and T. elongatus (PDB codes 6G08 and 6RNW respectively)(Extended Data Table 1a, Supplementary Methods), solved at $1.3 \AA$, 1.9 $\AA ̊$ and $1.9 \AA$ Å resolution, respectively (Fig. 2 and Extended Data Figure 1). As a member of the short-chain dehydrogenase and reductase (SDR) family of enzymes, the overall structure of POR is similar to that of other members of the SDR family ${ }^{19}$ and has a typical dinucleotide-binding Rossmann fold, which comprises a central $\beta$ sheet surrounded by six $\alpha$-helices (Extended Data Figs. 1, 2). Three flexible regions of the structure (residues 146-160 , 228-255 and 284-291; hereafter denoted R1, R2 and R3, respectively) are not observed in the T. elongatus electron density. This observation is consistent with molecular-dynamics simulations of both the apo- and coenzyme-bound structures (Extended Data Fig. 3), which indicates these regions have a high degree of flexibility. Despite this, both the R1 region and the and R2 region are ordered in the coenzyme-bound structure of Synechocystis POR and implicated in coenzyme binding to POR. A short loop (residues 223-229) within the $\mathrm{R} 2$ region extends from the central $\beta$-sheet, covers the nicotinamide moiety of the NADPH and becomes ordered upon binding of the coenzyme. A similar loop is observed over the coenzyme-binding pocket of other SDR enzymes, and it has preiously been shown that coenzyme binding causes the extended area around the loop to form two short $\alpha$-helices that act as a lid to cover the active site, which leads to a 'closed' conformation. ${ }^{20-23}$ In this region of Synechocystis POR, only one longer $\alpha$-helix (residues 230-239, hereafter referred to as helix 1)(Fig. 2a) is observed although an additional loop (residues 148-159, hereafter referred to as loop 1) (Fig. 
2a) which extends from the central $\beta$-sheet, is located near helix 1. It appears that loop 1, which is conserved in POR enzymes (Extended Data Fig. 1e, Supplementary Fig.1), and helix 1 are important in controlling the subsequent binding of the Pchlide substrate.

Beyond the loop regions, our POR crystal structures identify other residues that are important to coenzyme binding. The SDR family of proteins uses an Asn-Ser-Tyr-Lys catalytic tetrad for proton transfer and stabilization of reaction intermediates, ${ }^{19}$ however, in POR a Thr residue (Thr145) often replaces the Ser residue (Supplementary Fig. 1). Crystal structures of the binary POR-NADPH complex show that three of these residues - Asn90, Tyr193 and Lys197 (numbering refers to the $T$. elongatus POR), are directly hydrogen-bonded to the coenzyme (Fig. 2b, c). Also, Arg38, Lys42 and Asp63 interact with the coenzyme via a hydrogen-bonding network to the 2' phosphate of the molecule (Fig. 2b, c and Extended Data Fig. 4). Arg38 is implicated in coenzyme binding and release as it adopts alternative conformations in the different POR structures (Fig. 2d), a hypothesis that is further supported by the reduced NADPH binding affinity observed in an R38V variant. ${ }^{24} \mathrm{~A}$ water channel surrounding the Tyr193 and Lys197 residues of the active site is also identified in our structures, which could act as a potential proton relay network during catalysis (Fig. $2 \mathrm{e})$.

Despite extensive efforts, crystals of the ternary POR complex (comprising POR, Pchlide and $\mathrm{NADP}(\mathrm{H})$ ) were not obtained, as a result of the enzyme adopting an array of oligomeric states on binding the substrate (Supplementary Fig.2). We therefore used molecular docking to produce an initial model for the structure of the PORPchlide-NADPH ternary complex, followed by simulated annealing molecular dynamics simulations and umbrella sampling to calculate the potential of mean force for Pchlide binding (Extended Data Figs. 3,5). Although the potential of mean force changes over the time course of the simulation, the lowest energy conformation and the approximate dissociation energy remain stable and well-defined (Extended Data Fig. $5 \mathrm{~d}$, e). The resulting binding energy of about $35 \mathrm{kJmol}^{-1}$ (which corresponds to a dissociation constant of $0.8 \mu \mathrm{M}$ ) agrees well with the experimental dissociation 
constant of about $2 \mu \mathrm{M}$ (Extended Data Table 1b, Supplementary Fig. 3). The lowest energy conformation yields a ternary complex structure that is more rigid than the flexible, highly mobile structures that were observed for apo-POR and POR-NADPH (Extended Data Fig. 3a-c). In the ternary complex structure, helix 1 folds over the Pchlide and, together with loop 1, forms a lid over the active site (Fig.3a). The hydrophobic edge of Pchlide points towards hydrophobic regions of the enzyme (including the residues in helix 1) while the keto, methylester and carboxylic acid groups of the Pchlide molecule are positioned in a hydrophilic pocket (Fig. 3b). This provides numerous hydrogen-bonding opportunities and positions Pchlide in an orientation that is consistent with the required stereochemistry of hydride and proton transfer (Fig. 3c and Extended Data Fig. 5). Specific interactions include a salt bridge between Lys197 and the carboxylic acid side chain at the C-17 position, which also forms a hydrogen bond to Thr145. The central $\mathrm{Mg}^{2+}$ ion of Pchlide is ligated to two water molecules, one of which appears to be part of a water network that also involves hydrogen bonds to Tyr223 and the methylester at the C-15 position.

The photocatalytic implications of the determined structures and models of the active ternary complex were investigated using activity, binding and inhibition studies of site-directed variants and analogues of Pchlide with modifications to substituent groups that are of potential importance to enzyme mechanism (Extended Data Fig. 6, Extended Data Table 1b, Supplementary Figs. S3-8). Pchlide analogues were altered in the nature of the substituents on ring $\mathrm{E}$, the central $\mathrm{Mg}^{2+}$ ion and the carboxylic acid sidechain at the $\mathrm{C}-17$ position, all of which have previously been inferred as being important in POR-catalyzed photoreduction. ${ }^{25}$ An analogue in which the propionic acid sidechain at the $\mathrm{C}-17$ position is exchanged for a methylester group does not bind to POR. This analogue is also not a competitive inhibitor of the natural enzyme-catalyzed reaction. This emphasizes the importance of this propionic acid side chain in Pchlide binding to the POR active site, consistent with our structural models. Mutagenesis of Lys197 or Thr145 also leads to an impaired ability of POR to bind Pchlide substrate. ${ }^{24,26}$ This finding is consistent with the identified salt bridge and hydrogen bond made by these residues in their interaction with the propionic acid side chain of Pchilde. Additional Pchlide analogues modified at a rangeof locations of the porphyrin retain an ability to bind to the enzyme. These analogues also act as 
competitive inhibitors, albeit with affinities that are reduced compared to Pchlide itself (Extended Data Fig. 6, Extended Data Table 1b). Loss of the central $\mathrm{Mg}^{2+}$ ion and the keto group (C-13 position) led to about a 3-5-fold increase in $K_{\mathrm{d}}$, as expected from proposed roles in binding via coordination with water molecules in the active site. Although Lys 156 is situated in the loop region of the lid (in close proximity to Pchlide), activity measurements using a K156A variant indicate that Lys 156 is not important for binding (Extended Data Table 1b, Supplementary Fig. 8). This is consistent with the lack of hydrogen-bonding from Lys156 to Pchlide in the final model of the ternary complex. Molecular-dynamics simulations indicate Tyr223 facilitates the binding of Pchlide in the orientation that is required for catalysis via hydrogen bonding to the keto group at an intermediary phase in the binding process ( $R_{0}$ of about $17 \AA$ in the potential of mean force in Extended Data Figs. 5, 7) but does not directly interact with Pchlide in the ternary complex (Extended Data Fig. 6, Supplementary Video 1). This is consistent with experimental binding data where a significant reduction of about four- and tenfold is observed in the binding of Pchlide to Y223F and Y223A variants of the PORs (Extended Data Table 1b, Supplementary Fig. 8).

The POR crystal structures and ternary complex model now provide a structural basis to understand light-activated catalysis in POR. The extensive hydrogen-bonding network identified between active-site residues and Pchlide is likely to be essential for photochemistry, and these interactions are proposed to strengthen in the excited state to create an electron-deficient site at the C-17-C-18 double bond. ${ }^{9,10}$ The central $\mathrm{Mg}^{2+}$ ion and keto group at the $\mathrm{C}-13$ position of the Pchlide are also important to Pchlide photochemistry (=Extended Data Fig. 8, Supplementary Fig. 9), as they facilitate charge separation across the Pchlide during photoexcitation. ${ }^{11}$ The active-site architecture of POR (Extended Data Fig. 9) is finely tuned to facilitate this excitedstate charge separation and to stabilize the strengthened dipole across the Pchlide molecule (Fig. 4). The positive end of the dipole is located within the hydrophobic pocket of the active site, in which it is stabilized by $\pi$ - $\pi$ stacking interactions with a conserved Phe residue. By contrast, the negative end of the dipole is found in a region of polar residues. Interactions between Thr145 and Lys197of POR and the propionic acid side chain of Pchlide at C-17 are important to excited-state chemistry, as shown 
by the fact that changes to either residue results in impaired photochemistry. ${ }^{10,24,26}$ These excited-state interactions between Pchlide and POR stabilize a highly polarized C-17-C-18 double bond and enables stepwise hydride transfer from NADPH to C17. ${ }^{18}$ The polarized nature of the $\mathrm{C}-17-\mathrm{C}-18$ bond may also be stabilized by the close proximity of the hydroxyl group of Tyr193, which is known to be required for Pchlide photochemistry. ${ }^{10}$ By contrast, Tyr223 does not form any direct interaction with Pchlide (Extended Data Fig. 6, Extended Data Table 1b) and - consistent with this finding - the POR variants Y223A and Y223F are able to catalyze hydride transfer from NADPH to Pchlide (Supplementary Figs.10, 11). The donor-acceptor distance between the NADPH pro-S hydrogen and Pchlide C-17 is $4.5 \pm 0.3 \AA$ (Extended Data Fig. 6b), but this may change slightly upon photoexcitation of the Pchlide molecule. The subsequent transfer of a second proton to C-18 of Pchlide is from the strictly conserved Tyr193 of POR ${ }^{14,26}$ (Extended Data Fig. 6c) with a donor-acceptor distance of $4.9 \pm 0.4 \AA$ in our model - although this distance may change upon formation of a Pchlide anion following reduction of the C-17-C-18 double bond. In reactions catalyzed by other members of the SDR family, the catalytic Tyr is replenished through a proton relay mechanism. ${ }^{19}$ In the basis of the crystal structures reported here, the adjacent Lys197 is implicated in modulating the ionization properties of Tyr193. As the Y223A and Y223F variants of POR possess reduced rates of proton transfer (Supplementary Fig. 11), the water network coordinated by Tyr223 may also be important in this proton relay mechanism. The crystal structure of POR reported here should enable computational and time-resolved structural-mechanistic studies of the complete enzyme reaction cycle to provide the spatial, temporal and energetic understanding across multiple timescales (for example femtoseconds-to-seconds), and thereby address a major challenge in biological catalysis. 


\section{References}

1. Yang, J. \& Cheng, Q. Origin and evolution of the light-dependent protochlorophyllide oxidase (LPOR) genes. Plant Biol. 6, 537-544 (2004).

2. Scrutton, N.S., Groot, M.L., Heyes, D.J. Excited state dynamics and catalytic mechanism of the light-driven enzyme protochlorophyllide oxidoreductase. Phys Chem Chem Phys 14, 8818-8824 (2012).

3. Gabruk, M. \& Mysliwia-Kurdziel, B. Light-dependent protochlorophyllide oxidoreductase: phylogeny, regulation, and catalytic properties. Biochemistry 54, 5255-5262 (2015).

4. Aubert, C., Vos, M. H., Mathis, P., Eker, A. P. \& Brettel, K. Intraprotein radical transfer during photoactivation of DNA photolyase. Nature 405, 586-590 (2000).

5. Sorigue, D. et al An algal photoenzyme converts fatty acids to hydrocarbons. Science 357, 903-907 (2017).

6. Dietzek, B. et al Excited-state processes in protochlorophyllide a a femtosecond time-resolved absorption study. Chem. Phys. Lett. 397, 110-115 (2004).

7. Dietzek, B. et al Dynamics of charge separation in the excited-state chemistry of protochlorophyllide. Chem. Phys. Lett. 492, 157-163 (2010).

8. Sytina, O.A. et al Protochlorophyllide excited-state dynamics in organic solvents studied by time-resolved visible and mid-infrared spectroscopy. $J$. Phys. Chem. B 114, 4335-4344 (2010).

9. Zhao, G.J., Han, K.L. Site-specific solvation of the photoexcited protochlorophyllide a in methanol: formation of the hydrogen-bonded intermediate state induced by hydrogen-bond strengthening. Biophys. J. 94, 3846 (2008).

10. Heyes, D.J. et al Excited state charge separation in the photochemical mechanism of the light-driven enzyme protochlorophyllide oxidoreductase. Angewandte Chemie Intl. Ed. 54, 1512-1515 (2015). 
11. Heyes, D.J. et al Excited-State Properties of Protochlorophyllide Analogues and Implications for Light-Driven Synthesis of Chlorophyll. J. Phys. Chem. B., 121, 1312-1320 (2017).

12. Brandariz-de-Pedro, G. et al Direct Evidence of an Excited-State Triplet Species upon Photoactivation of the Chlorophyll Precursor Protochlorophyllide. J. Phys. Chem. Lett. 8, 1219-81223 (2017).

13. Heyes, D.J. et al. The first catalytic step of the light-driven enzyme protochlorophyllide oxidoreductase proceeds via a charge transfer complex. $J$. Biol. Chem. 281, 26847-26853 (2006).

14. Heyes, D.J., Sakuma, M., de Visser, S., Scrutton, N.S. Nuclear quantum tunneling in the light-activated enzyme protochlorophyllide oxidoreductase. $J$. Biol. Chem. 284, 3762-3767 (2009).

15. Heyes, D. J., Sakuma, M. \& Scrutton, N. S. Solvent-slaved protein motions accompany proton but not hydride tunneling in light-activated protochlorophyllide oxidoreductase, Angew. Chem. Int. Edit. 48, 3850-3853 (2009).

16. Heyes, D. J., Levy, C., Sakuma, M., Robertson, D. L. \& Scrutton, N. S. A Twin-track Approach Has Optimized Proton and Hydride Transfer by Dynamically Coupled Tunneling during the Evolution of Protochlorophyllide Oxidoreductase. J. Biol. Chem. 286, 11849-11854 (2011).

17. Hoeven, R., Hardman, S. J. O., Heyes, D. J. \& Scrutton, N. S. Cross-species analysis of protein dynamics associated with hydride and proton transfer in the catalytic cycle of the light-driven enzyme protochlorophyllide oxidoreductase, Biochemistry 55, 903-913 (2016).

18. Archipowa, N., Kutta, R.J., Heyes, D.J. \& Scrutton, N.S. Stepwise Hydride Transfer in a Biological System: Insights into the Reaction Mechanism of the Light-Dependent Protochlorophyllide Oxidoreductase. Angew. Chem. Int. Ed. 57, 2682-2686 (2018).

19. Kavanagh, K. L., Jornvall, H., Persson, B. \& Oppermann, U. Medium- and short-chain dehydrogenase/reductase gene and protein families : the SDR 
superfamily: functional and structural diversity within a family of metabolic and regulatory enzymes. Cell Mol. Life Sci. 65, 3895-3906 (2008).

20. Korman, T. P., Tan, Y. H., Wong, J., Luo, R. \& Tsai, S. C. Inhibition kinetics and emodin cocrystal structure of a type II polyketide ketoreductase.

Biochemistry 47, 1837-1847 (2008).

21. Javidpour, P. et al. The Determinants of Activity and Specificity in Actinorhodin Type II Polyketide Ketoreductase. Chem. Biol. 20, 1225-1234 (2013).

22. Blaise, M., Van Wyk, N., Baneres-Roquet, F., Guerardel, Y. \& Kremer, L. Binding of $\mathrm{NADP}(+)$ triggers an open-to-closed transition in a mycobacterial FabG beta-ketoacyl-ACP reductase. Biochem. J. 474, 907-921 (2017).

23. Zhao, F. J. et al. Crystal structure and iterative saturation mutagenesis of ChKRED20 for expanded catalytic scope. Appl. Microbiol. Biotech. 101, 83958404 (2017).

24. Menon, B.R.K., Hardman, S.J.O., Scrutton, N.S. \& Heyes, D.J. Multiple active site residues are important for photochemical efficiency in the light-activated enzyme protochlorophyllide oxidoreductase (POR). J. Photochem. Photobiol. B. 161, 236-243 (2016).

25. Klement, H., Helfrich, M., Oster, U., Schoch S., Rudiger, W. Pigment-free NADPH:protochlorophyllide oxidoreductase from Avena sativa L. Eur. J. Biochem. 265, 862. (1999).

26. Menon, B.R.K., Waltho, J.P., Scrutton, N.S., Heyes, D.J. Cryogenic and laser photoexcitation studies identify multiple roles for active site residues in the light-driven enzyme protochlorophyllide oxidoreductase. J. Biol. Chem. 284, 18160-18166 (2009). 


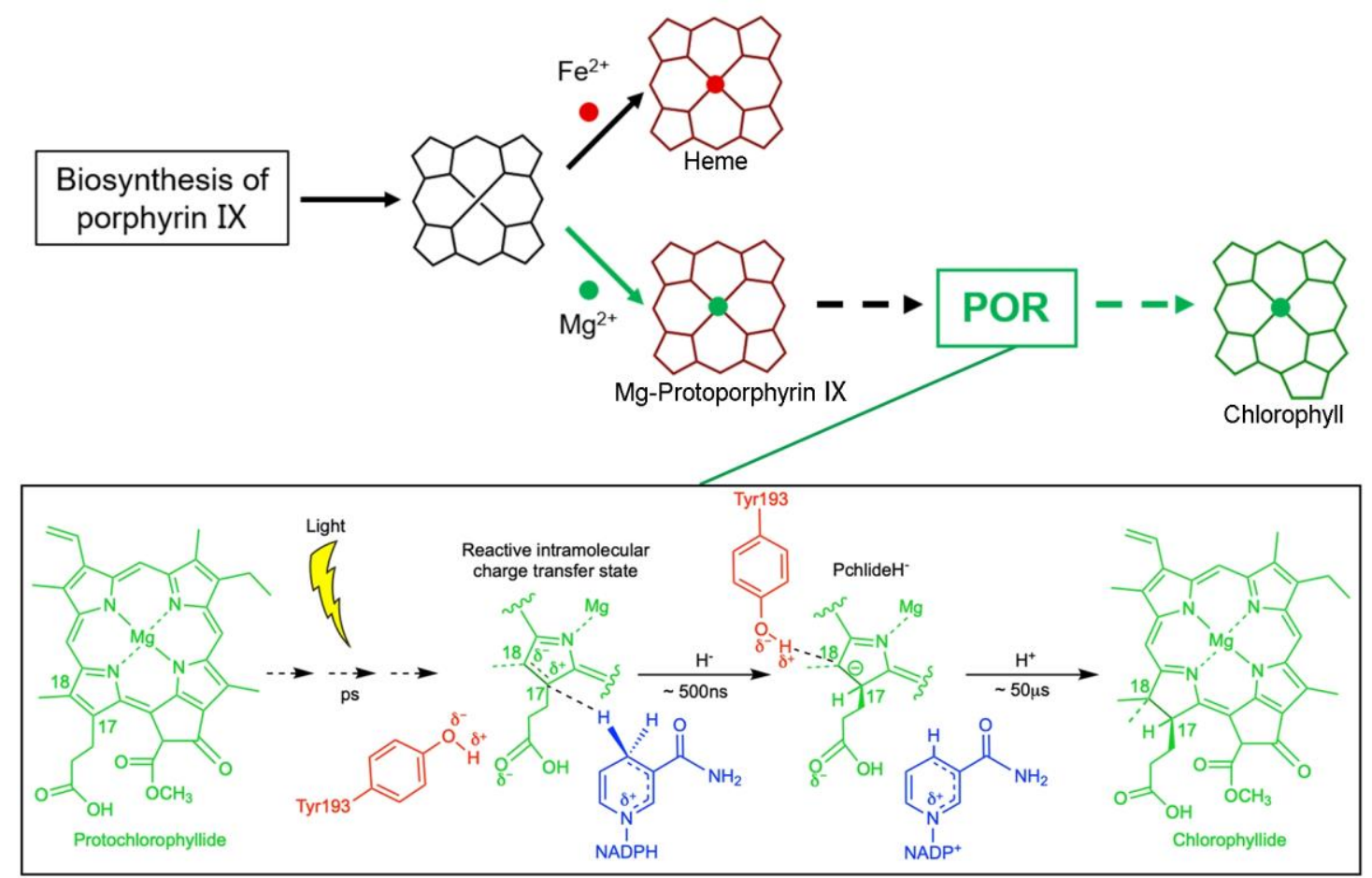

Figure 1. The light-driven reduction of the C-17-C-18 double bond of Pchlide to

form chlorophyllide. The reaction is catalysed by POR and is a key regulatory step within the chlorophyll biosynthetic pathway. Catalysis involves excited-state interactions between the Pchlide and protein, which lead to sequential hydride transfer from NADPH to the $\mathrm{C}-17$ position and proton transfer to the $\mathrm{C}-18$ position. 

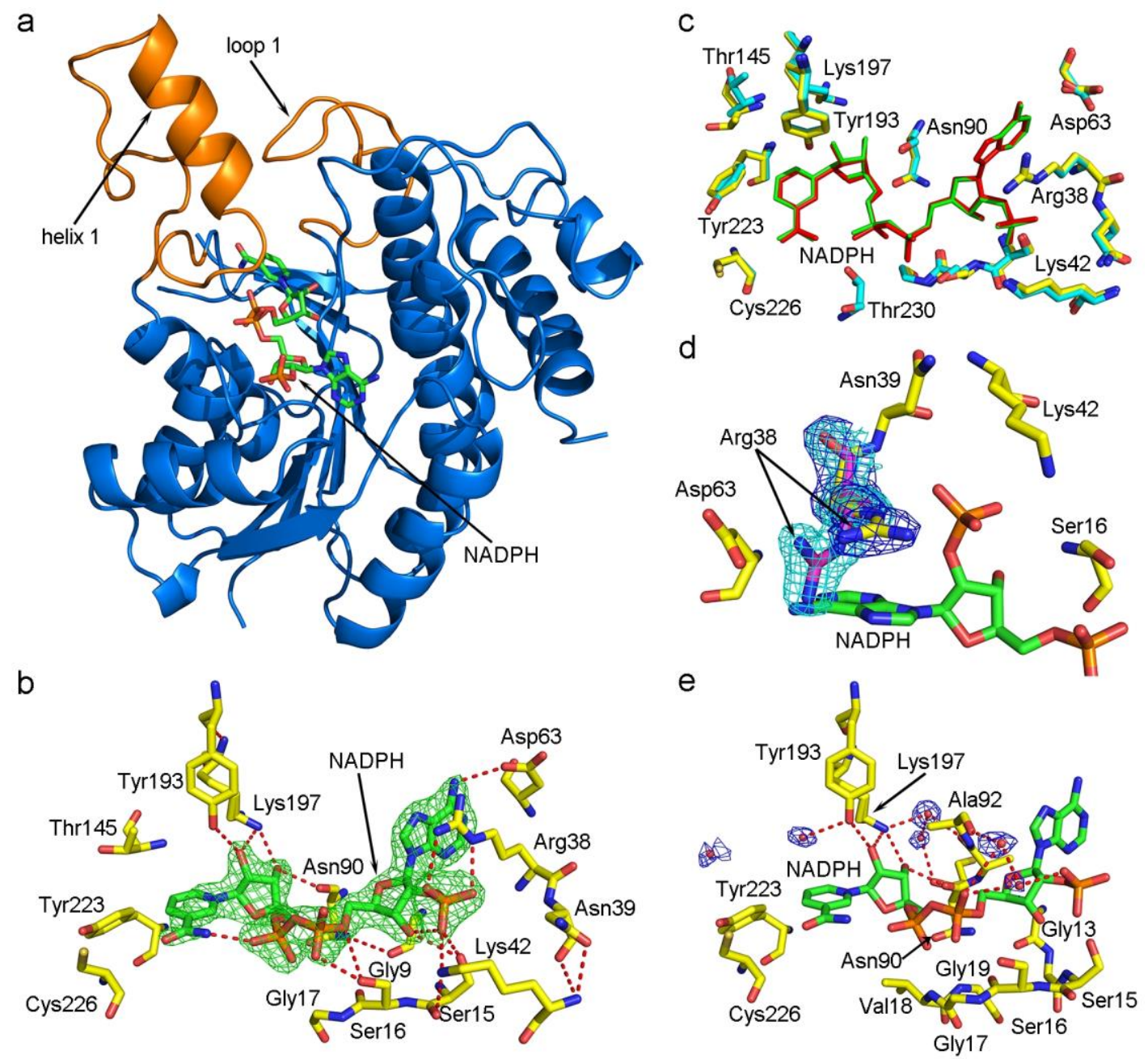

Figure 2. Crystal structures of light-dependent POR. a, Synechocystis POR crystal structure with NADPH bound in the active site (Supplementary Methods). The regions colored orange are the missing loops in T. elongatus POR, which are present in the crystal structure of Synechocystis POR. b, Structure of the coenzyme-binding site of T. elongatus POR. Hydrogen bonds between key residues and NADPH are shown as red dashes. The electron density for NADPH (omit $2 \mathrm{~F}_{\mathrm{o}}-\mathrm{F}_{\mathrm{c}}$ map contoured at $1 \sigma$ ) is colored green. $\mathbf{c}$, Comparison of the coenzyme-binding site of T. elongatus POR and Synechocystis POR. The NADPH is colored as green (T. elongatus) or red (Synechocystis) stick. d, Comparison of the conformation of Arg38 in apo- and NADPH-bound structures of T. elongatus POR. The electron density for $\operatorname{Arg} 38\left(2 \mathrm{~F}_{\mathrm{o}^{-}}\right.$ $\mathrm{F}_{\mathrm{c}}$ map contoured at $1 \sigma$ ) in the apo-POR structure is colored blue; In the NADPHPOR complex, Arg38 is colored cyan. e, Potential water channel surrounding key residues in NADPH-bound structure of T. elongatus POR. Water molecules are 
shown as red balls, and hydrogen bonds between key residues and water molecules are shown as red dashes.
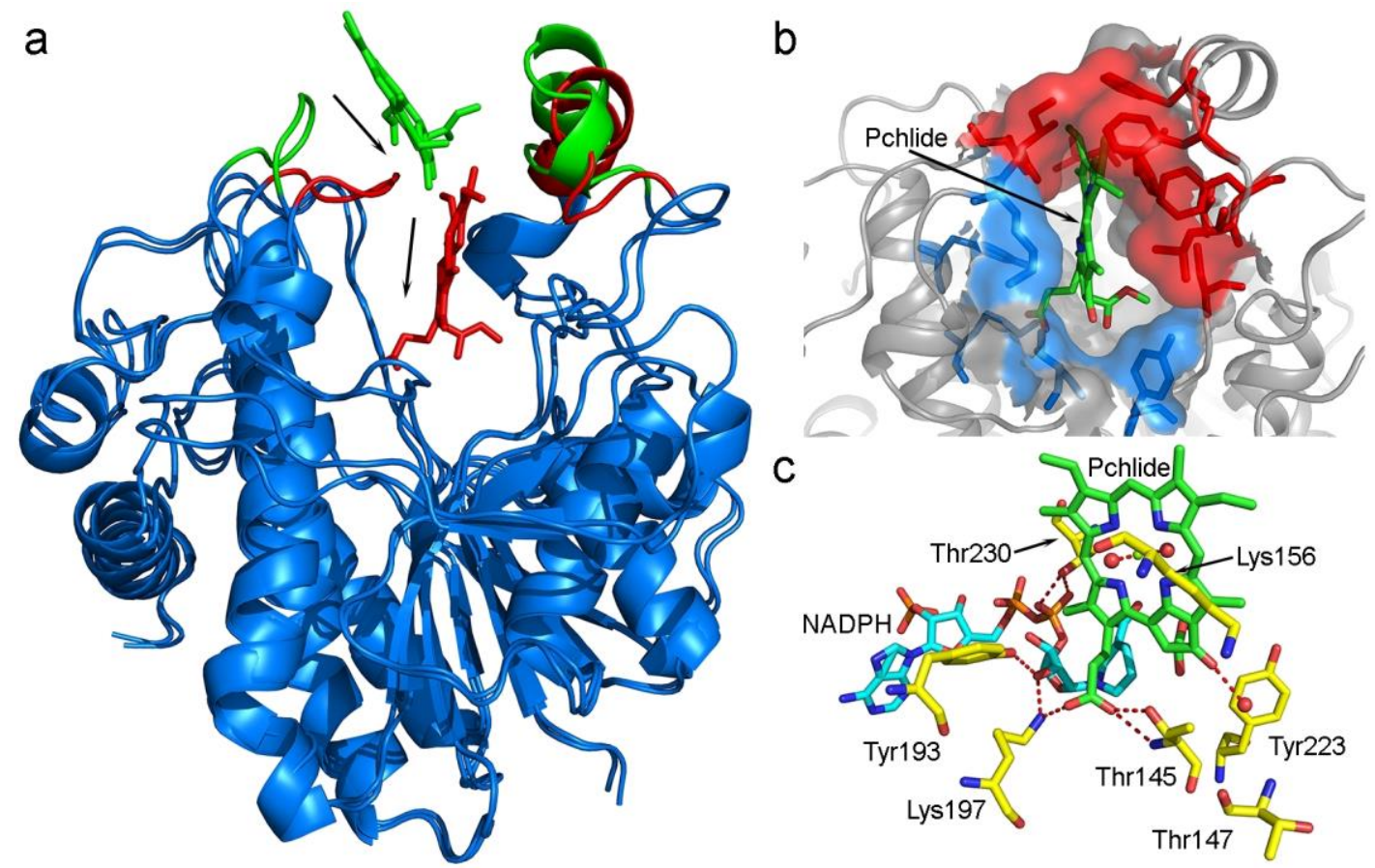

Figure 3. Structural model of the POR-Pchlide-NADPH ternary complex. a, The overall structure of the POR-Pchlide-NADPH ternary complex showing the structural changes upon Pchlide binding. The loop, helix and Pchlide molecule colored in green show the Pchlide entering the binding pocket and loop, helix and Pchlide molecule colored in red show the final binding position of Pchlide. b, View of the Pchlide binding pocket. The red surface represents hydrophobic residues and the blue surface represents hydrophilic residues. c, Structure of active site of the T. elongatus POR showing the hydrogen-bonding network around the Pchlide molecule. Water molecules are shown as red balls; potential hydrogen bonds are shown as red dashes. 


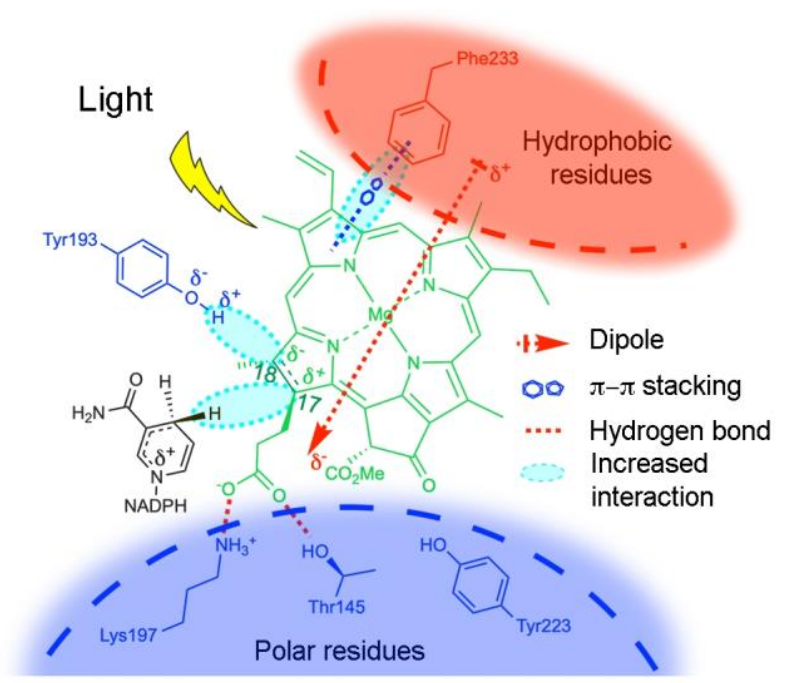

Figure 4. A structural basis for light-dependent reaction chemistry in POR.

Mechanistic scheme that illustrates potential structural reorganization of Pchlidebinding site upon excitation.

\section{Acknowledgements.}

The work was funded by the Engineering and Physical Sciences Research Council (fellowship to N.S.S.; EP/J020192/1). We thank Diamond Light Source beamlines I03 \& I04 (proposal numbers MX8997-35, MX12788-8, 19, 42 \& 62) and Shanghai Synchrotron Radiation Facility beamlines BL17U1 and BL19U1 for assistance during data collection. Time-resolved visible absorption measurements were performed using instrumentation funded by BBSRC Alert14 Award BB/M011658/1. This work was also supported by MOST 973 Project Biological Nitrogen Fixation (2010CB126504), National Basic Research Program of China (2014CB910304) and National Natural Science Foundation of China (31230004 and 81572090). We thank Ray Dixon (John Innes Centre, Norwich, UK) and Randy Read (University of Cambridge, UK) for early discussions and support. We finally thank C.E.Bauer and Y.Fujita for kindly providing the strain ZY5 for the production of Pchlide.

Author Contributions: D.J.H, A.Z., Q.C. and N.S.S. initiated and coordinated the project. S.Z., Q.C., A.Z., N.S.S., D.L. and D.J.H designed experiments, analysed data and wrote the manuscript with contributions from other authors. S.Z., M.S., L.F., W.S., H.L., J.Y., and X.L. produced and crystallized the proteins. A.Z. and C.W.L. 
collected and processed diffraction data and solved the structures. S.H. and L.O.J. performed the docking and molecular dynamics simulations. S.J.O.H. and D.J.H. performed time-resolved spectroscopy measurements. R.H. and M.S. assisted with protein purification and characterisation of POR variants. X.Y., M.L. and Z.R., advised on all aspects. All authors discussed the results and commented on the manuscript.

Author Information. The authors declare there are no financial or non-financial competing interests. Correspondence and request for materials should be addressed to A.Z., Q.C. or N.S.S.

Data availability. The atomic coordinates and experimental data have been deposited in the Protein data Bank with accession codes 6G08, 6RNV and 6RNW.All other data are available from the corresponding authors on reasonable request. 


\section{Extended data figures}

a
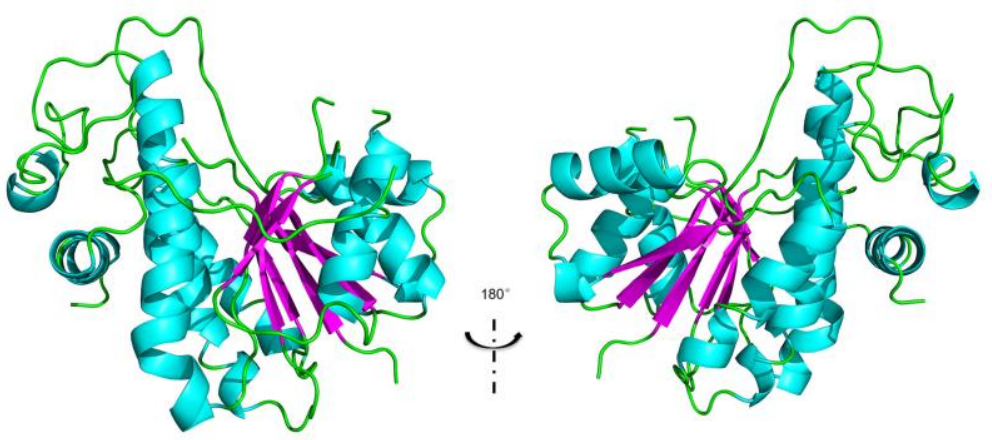

b
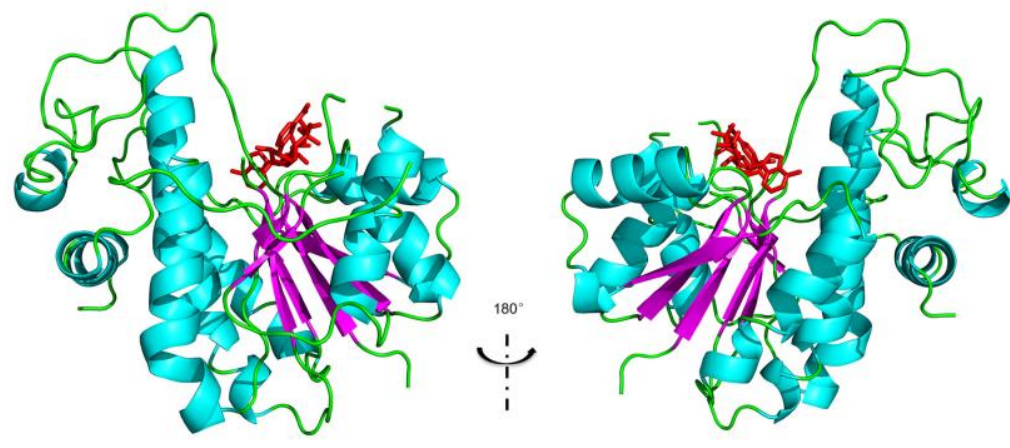

C
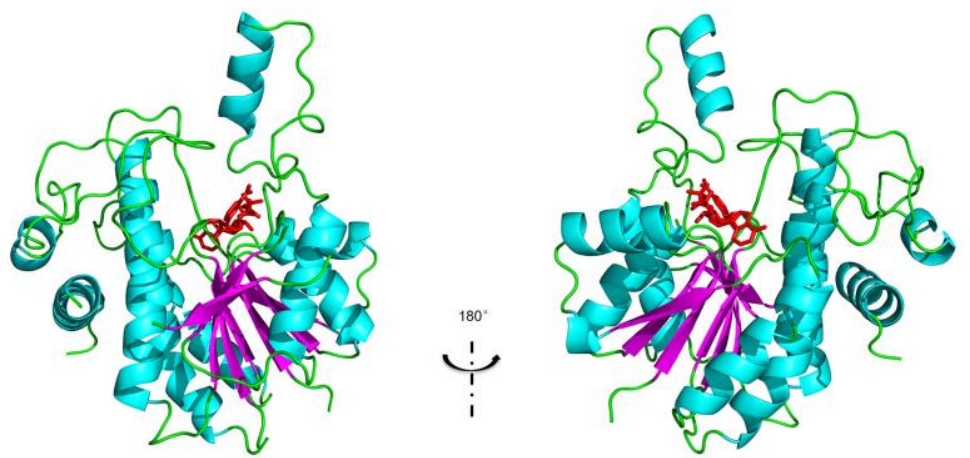

d

e
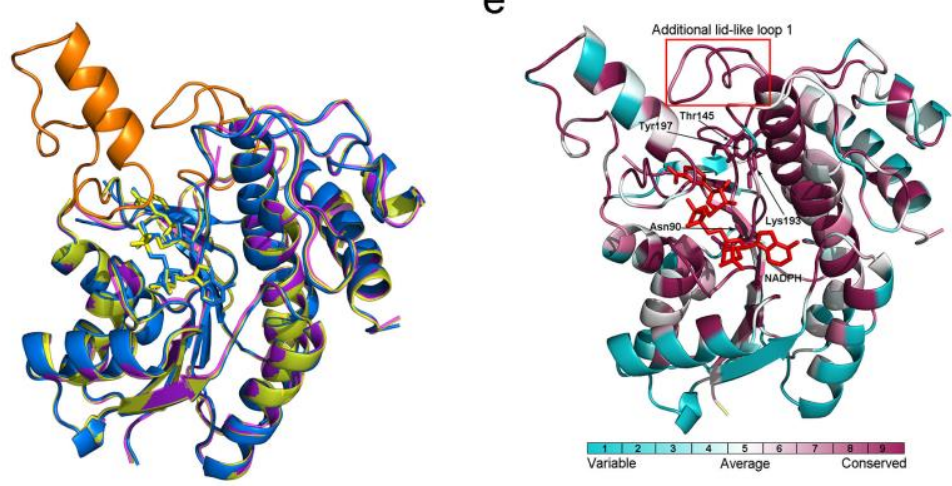

Figure 1. Crystal structures of T. elongatus and Synechocystis POR. (a) T. elongatus apo-POR. (b) T. elongatus POR with bound NADPH. (c) Synechocystis POR with bound NADPH. The protein structure is colored according to secondary structure, $\alpha$-helix cyan, $\beta$-sheet magenta and loop green. (d) Alignment of the 
different POR crystal structures solved in the present work. Magenta, apo structure of T. elongatus POR; yellow, structure of NADPH-bound T. elongatus POR; blue, structure of NADPH-bound Synechocystis POR. The regions colored orange are the missing loops in T. elongatus POR, which are present in the crystal structure of Synechocystis POR. (e) Evolutionary conservation of Synechocystis POR structure. Based on the phylogenetic relations between homologous sequences, evolutionary conservation of amino acid positions in Synechocystis POR protein has been performed through the online ConSurf server. ${ }^{39}$ 
a
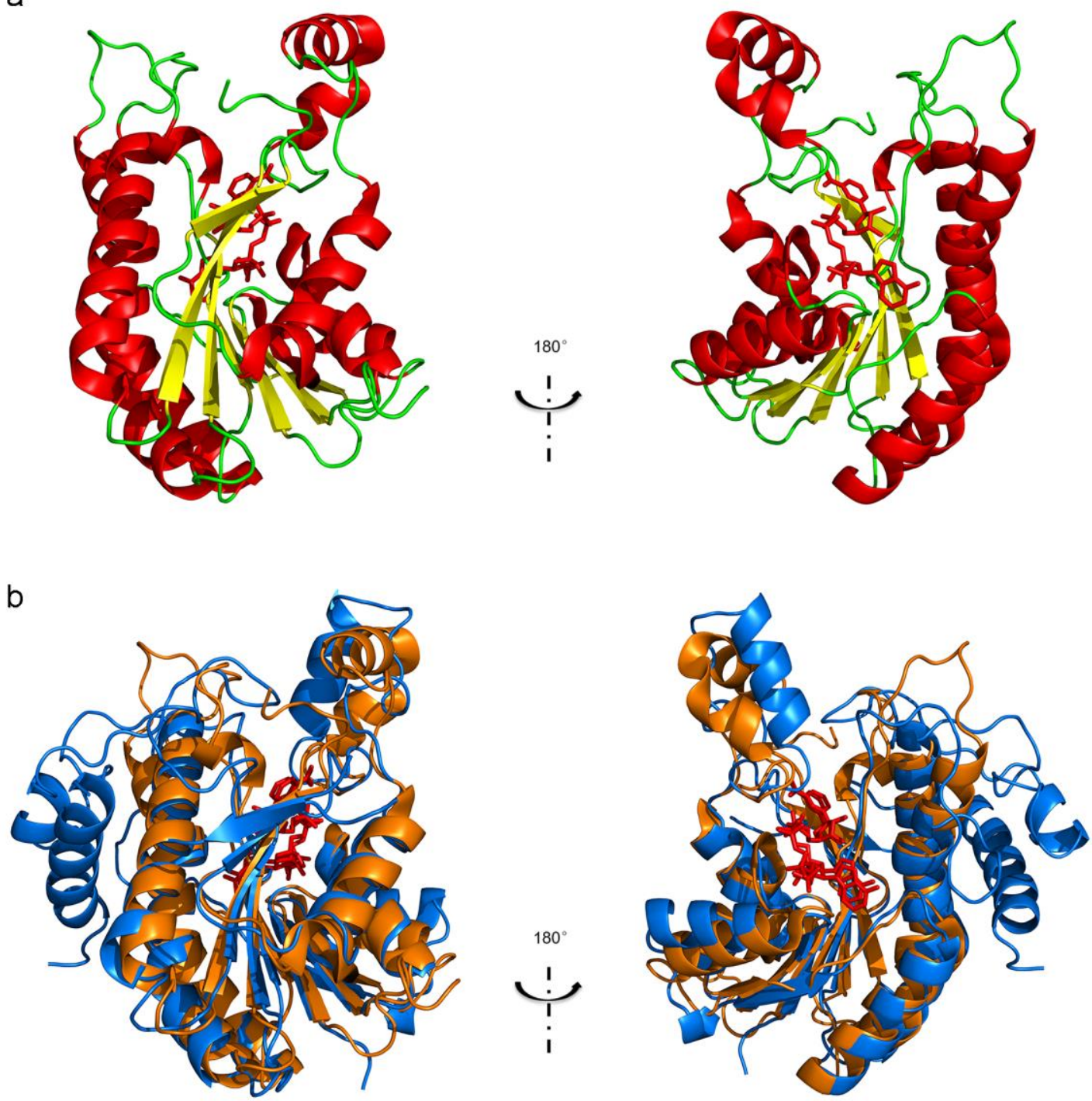

Figure 2. Comparison of Synechocystis POR structure with SDR family protein

及-ketoacyl reductase (PDB: 2B4Q). (a) Overall structure of $\beta$-ketoacyl reductase protein (colored by secondary structure). (b) Alignment of Synechocystis POR (blue) and $2 \mathrm{~B} 4 \mathrm{Q}$ (orange). NADPH is shown as a red stick. 

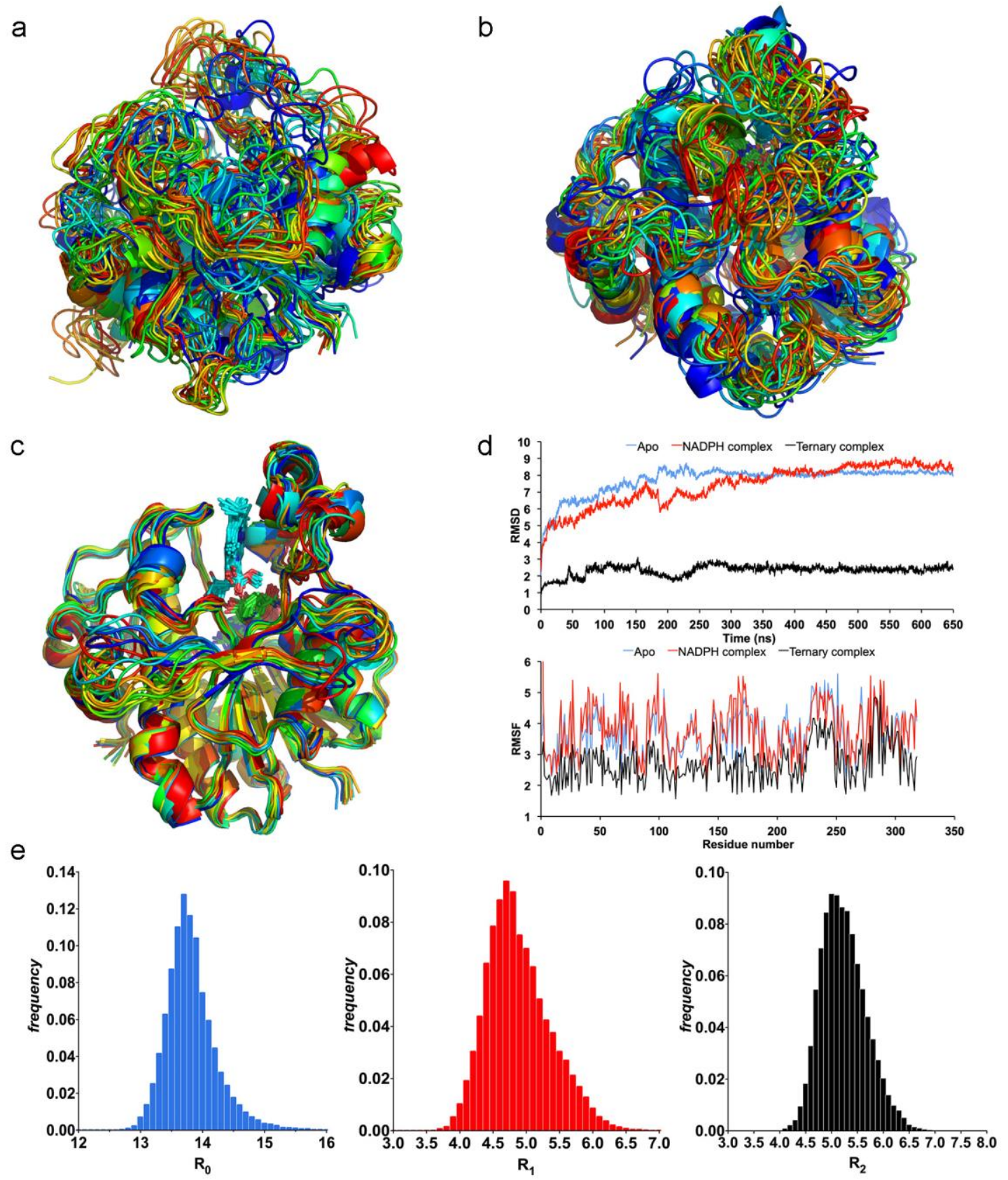

Figure 3. Molecular dynamics simulations of T. elongatus POR. Overlays of structures from unrestrained MD simulations at 500 ps intervals for (a) apo enzyme, (b) NADPH complex and (c) ternary complex. (d) RMSD (root-mean-squared deviation) vs. time and per-residue RMSF (root-mean squared fluctuation), calculated for the protein non-hydrogen atoms. (e) Distance distributions for the PChlide binding coordinate $R_{0}$ and the distances between the hydride donor and acceptor atoms $\left(R_{1}\right)$ and the proton donor and acceptor atoms $\left(R_{2}\right)$. 

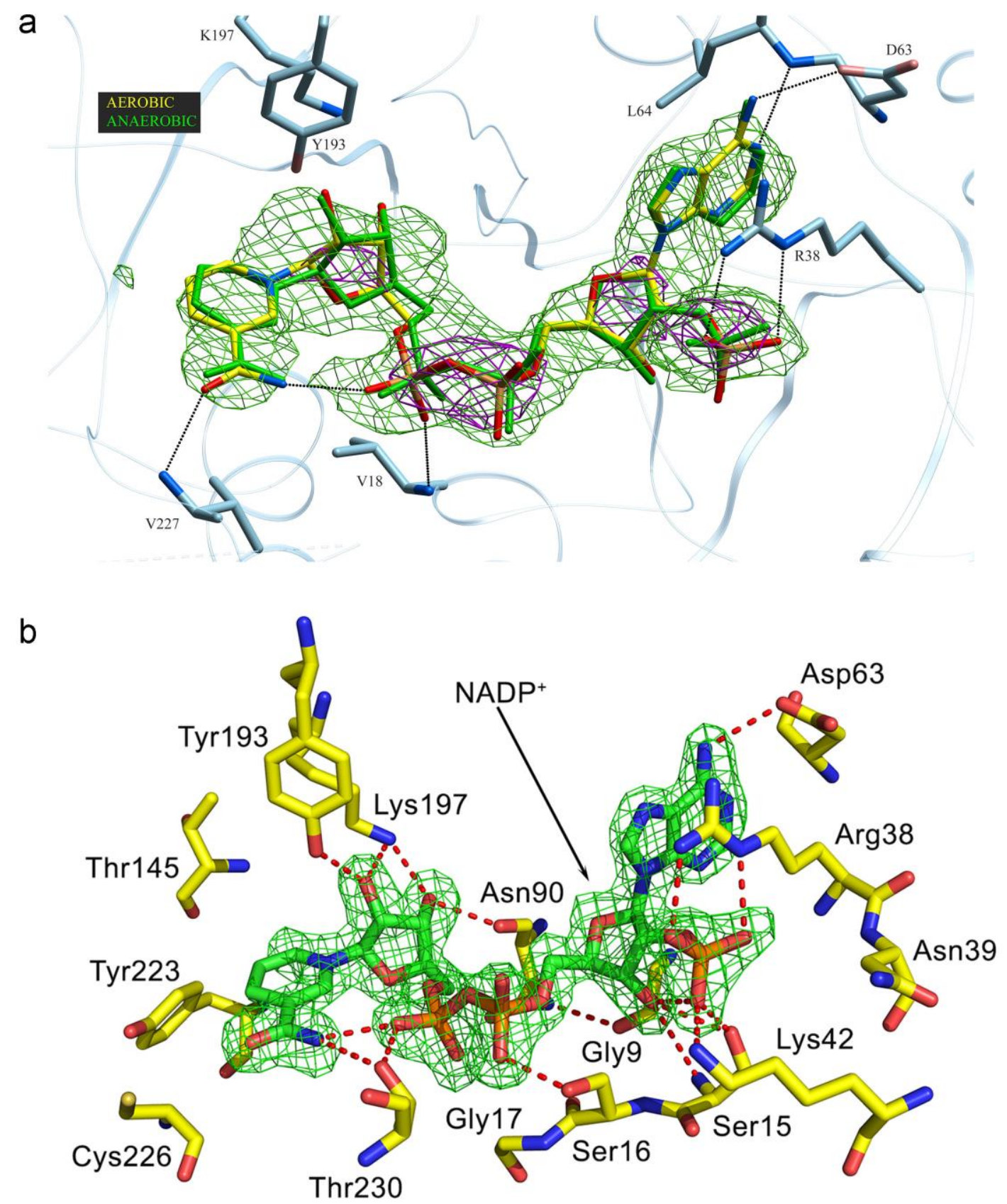

Figure 4. Electron density map for NADPH bound to T. elongatus and

Synechocystis POR. (a) NADPH anaerobic soaking with T. elongatus POR crystal. A low resolution $3.5 \AA$ electron density map (FEM) contoured at 1 sigma (green mesh) along with an Fo-Fc omit map contoured at 4 sigma (magenta mesh) is shown for the NADPH region of an anaerobically soaked T. elongatus POR crystal. An all atom coloured stick representation of the aerobically soaked high resolution NADPH-POR structure and associated active site interactions are shown along with a stick representation of the anaerobically soaked NADPH in green. (b) Structure of the NADPH binding site of Synechocystis POR. Hydrogen bonds between key residues 
and NADPH are shown as red dashes. The electron density for NADPH (omit Fo-Fc map contoured at $3 \sigma$ ) is colored green.
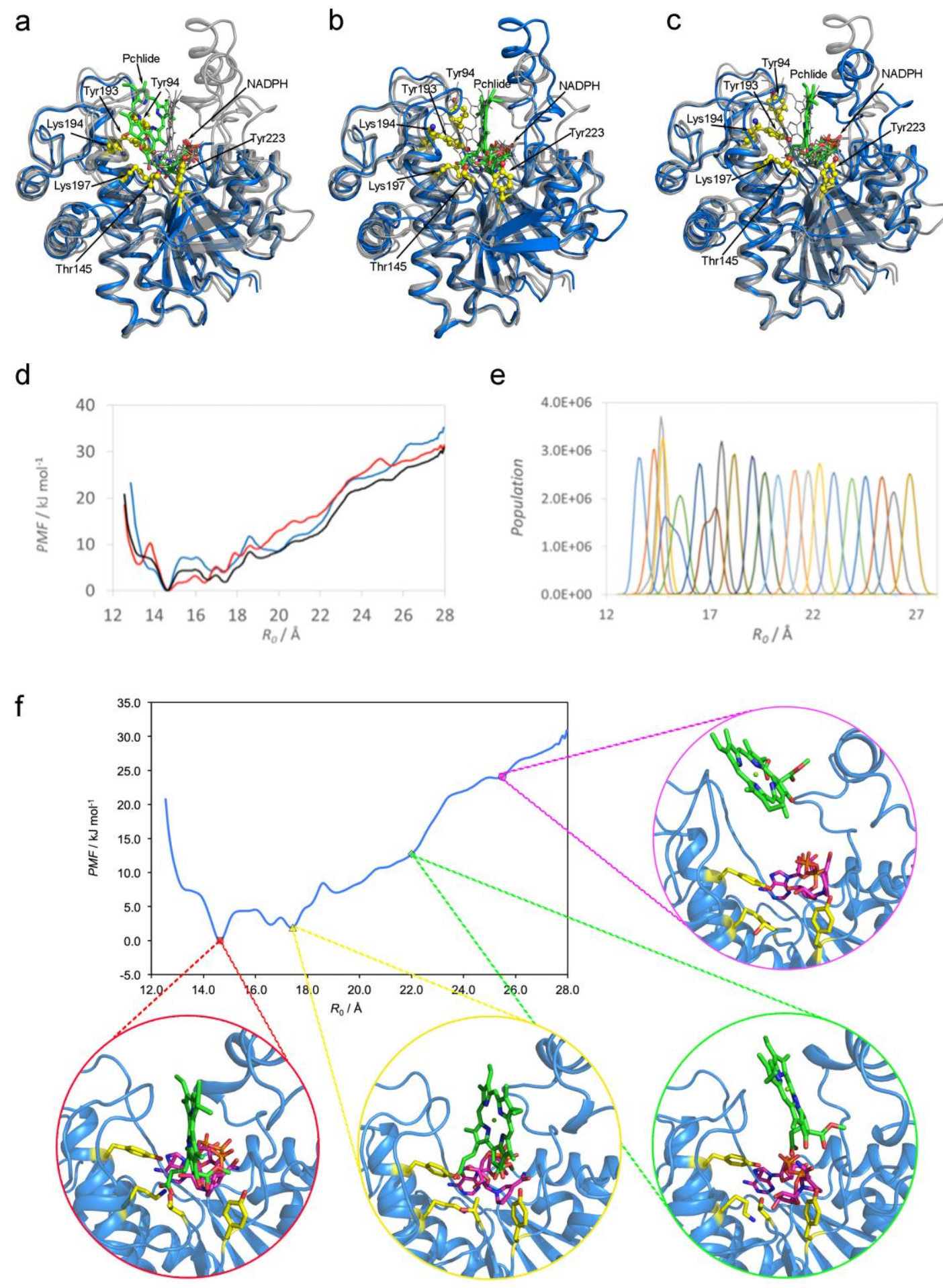
Figure 5. Modelling of the T. elongatus-Pchlide-NADPH ternary complex. Three stages of modelling the T. elongatus-Pchlide-NADPH ternary complex. The structures are aligned and superimposed, with each panel highlighting a different structure in blue (the other two in grey): (a) crystal structure with the chosen docked Pchlide structure; (b) the structure after 20 ns annealing; (c) the final representative structure. The flexible residues during docking are shown in yellow ball and stick representation and the Pchlide molecule is shown in green stick representation. (d) PMF calculated by umbrella sampling as a function of $R_{0}$ (see Methods) for $50 \mathrm{~ns}$ umbrella sampling (black) as well as the first $20 \mathrm{~ns}$ (blue) and the last $20 \mathrm{~ns}$ (red). (e) Population distributions for each bin, each sampled for 50 ns. (f) PMF was calculated by umbrella sampling as a function of $R_{0}$ (distance between Pchlide $\mathrm{Mg}^{2+}$ and lower edge of the POR binding pocket; see Methods). The dip in the PMF at $R_{0} \approx 17 \AA$ corresponds to the formation of a hydrogen bond between Y223 and the Pchlide keto group. 
a

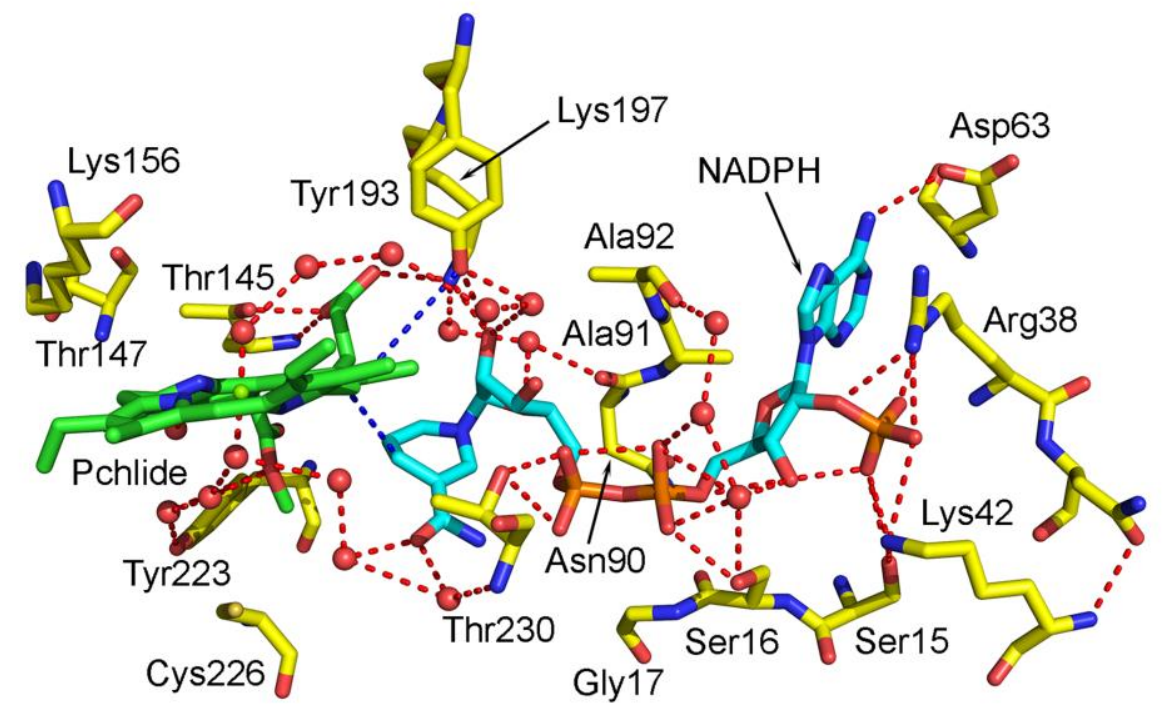

b
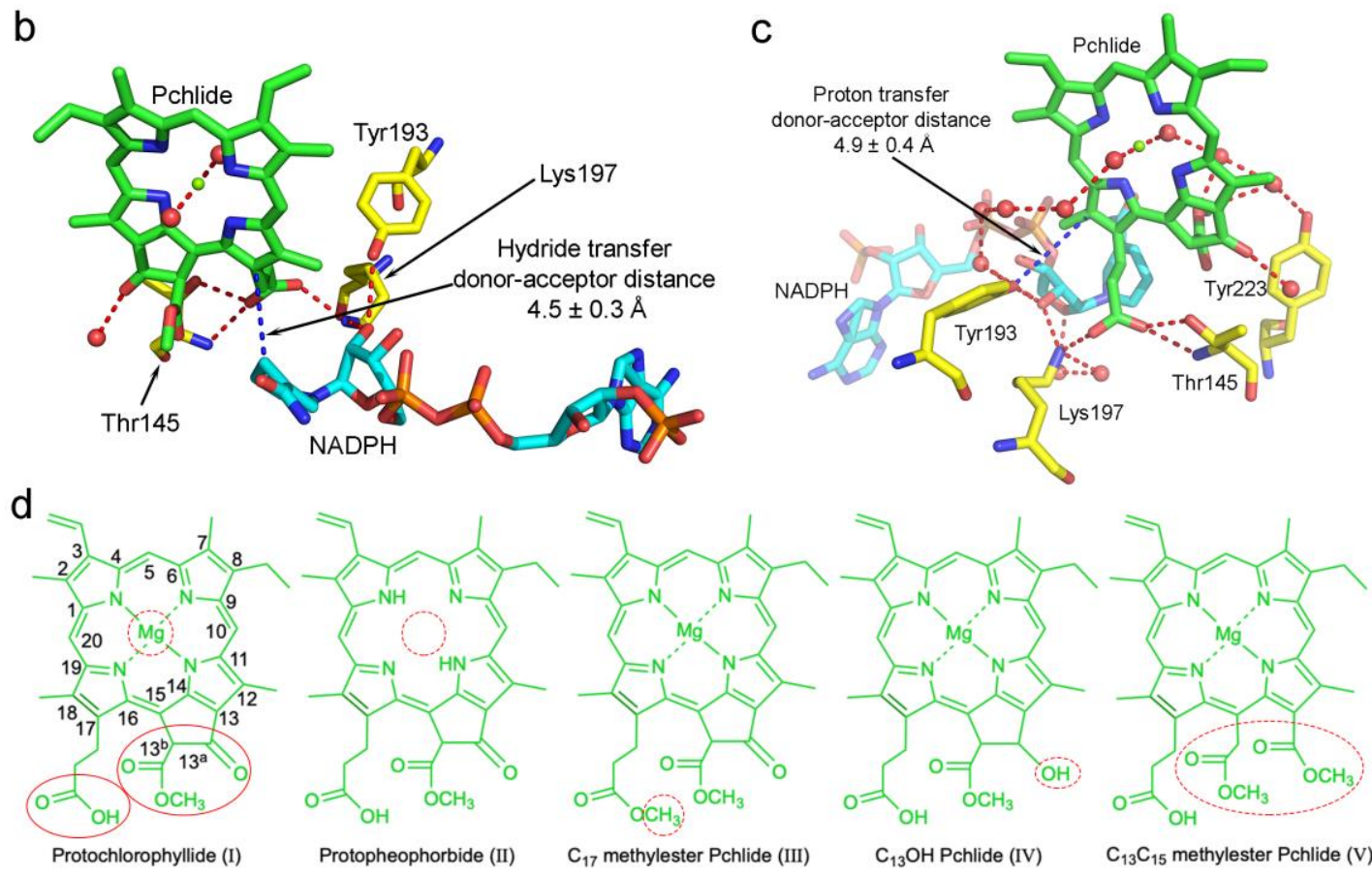

$k_{\text {cat }}=0.023 \pm 0.001 \mathrm{~s}^{-1}$

$K_{\mathrm{m}}=3.6 \pm 0.4 \mu \mathrm{M}$

Inactive

$\mathrm{C}_{17}$ methylester Pchlide (III)

$\mathrm{C}_{13} \mathrm{OH}$ Pchlide (IV)

Inactive

$K_{\mathrm{i}}=9.7 \pm 1.1 \mu \mathrm{M}$

$K_{\mathrm{d}}=6.1 \pm 1.2 \mu \mathrm{M}$
$\mathrm{C}_{13} \mathrm{C}_{15}$ methylester Pchlide (V)
$K_{\mathrm{i}}=50.8 \pm 3.6 \mu \mathrm{M}$
$K_{\mathrm{d}}=46.7 \pm 4.6 \mu \mathrm{M}$

$K=\mathrm{n} / \mathrm{a}$

$K_{d}=n / a$

$K_{\mathrm{d}}=9.7 \pm 3.5 \mu \mathrm{M}$

Figure 6. Configuration of the active site and donor-acceptor distance of the $T$. elongatus POR-NADPH-Pchlide ternary complex model. (a) Active site of the $T$. elongatus POR-NADPH-Pchlide ternary complex. The hydrogen bonding network around the Pchlide and NADPH molecules are shown as red dashes. The donoracceptor distance for hydride/proton transfer is shown as blue dashes. Water molecules are shown as red balls. (b) View of the active site of T. elongatus POR highlighting the donor-acceptor distance for hydride transfer (shown as blue dash). (c) View of the active site of T. elongatus POR highlighting the donor-acceptor distance 
for proton transfer (shown as blue dash). (d) Summary of the activity, binding and inhibition data for the Pchlide analogues. The structures and apparent $k_{\mathrm{cat}}, K_{\mathrm{m}}, K_{\mathrm{d}}$ and $K_{\mathrm{i}}$ (where applicable in each case) are shown for Pchlide (I), protopheophorbide (II), C17 methylester Pchlide (III), C13OH Pchlide (IV) and C13C15 methylester Pchlide (V). The red circles show the regions of the Pchlide molecule that have previously been shown to be important for activity (central $\mathrm{Mg}$, ring $\mathrm{E}$ and the side chain at the C17 position). The structures of all of the Pchlide derivatives described in the present study are shown with the modifications indicated by dashed red circles. 

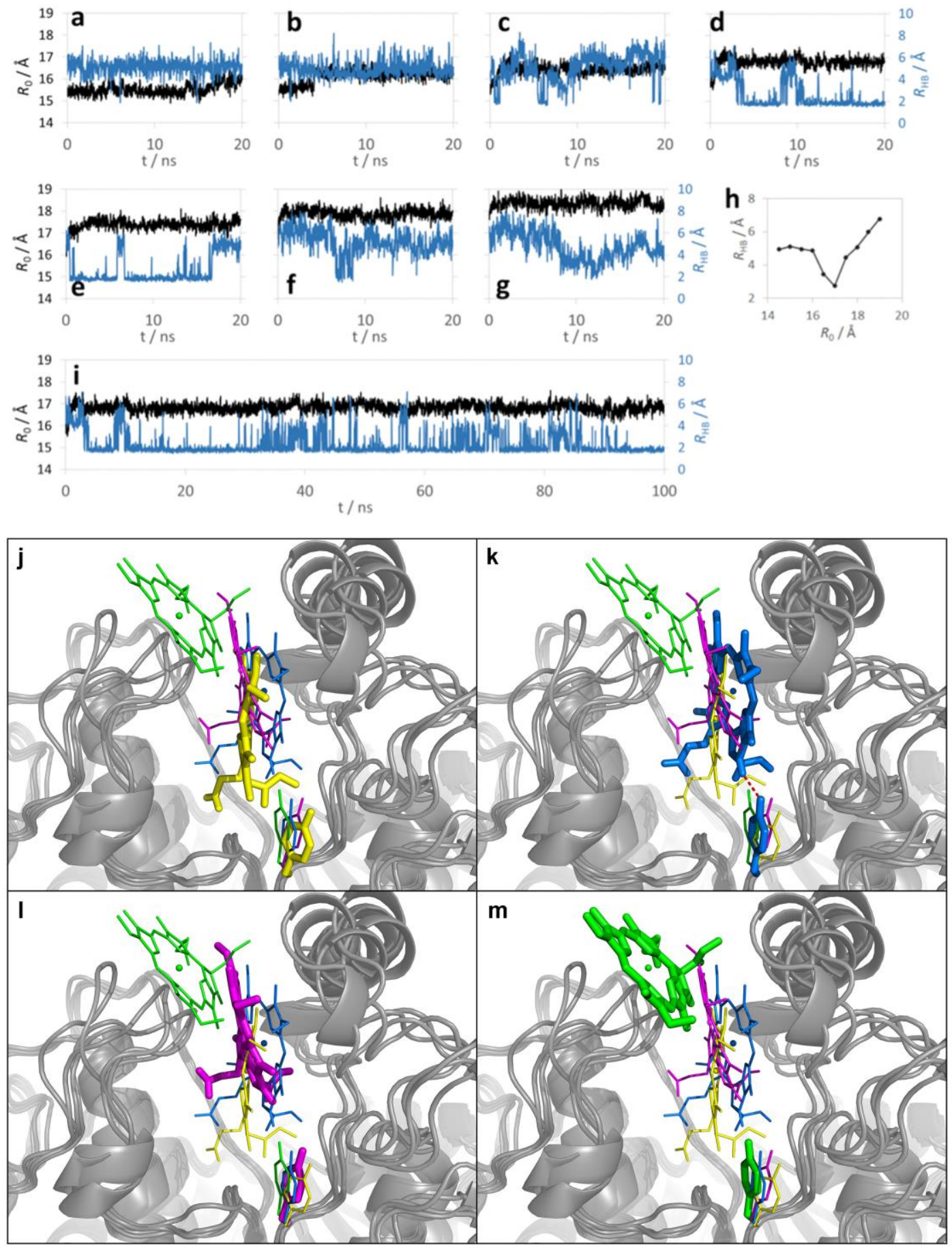

Figure 7. Hydrogen bonding interactions between Tyr223 and the C13 keto group during Pchlide binding. Change in hydrogen bonding between Tyr223 and Pchlide C13 keto group during Pchlide binding. (a-g). Plots of the distance $R_{0}$ between Pchlide $\mathrm{Mg}^{2+}$ and base of POR binding pocket (black) and the distance $R_{\mathrm{HB}}$ between the Tyr223 hydroxy proton and keto oxygen (blue) from $20 \mathrm{~ns}$ MD simulations at increasing $R_{0}$ values; (h) average $R_{\mathrm{HB}}$ for each $0.5 \AA$ bin for $R_{0}$; (i) extended $100 \mathrm{~ns}$ MD simualation with $R_{0}$ restrained at $1.7 \AA$ to further illustrate the 
stability of the hydrogen bond between Y223 and keto group. As the Pchlide leaves the binding pocket (from $\mathbf{j}$ to $\mathbf{m}$ ), the protein backbone is shown as a gray cartoon, the Tyr 223 residue is free to move around and form a transient hydrogen bond with the C13 keto group of Pchlide to 'guide' the Pchlide into its final orientation. At each stage the Pchlide and Y223 molecules have been highlighted with thicker sticks in the figure. 

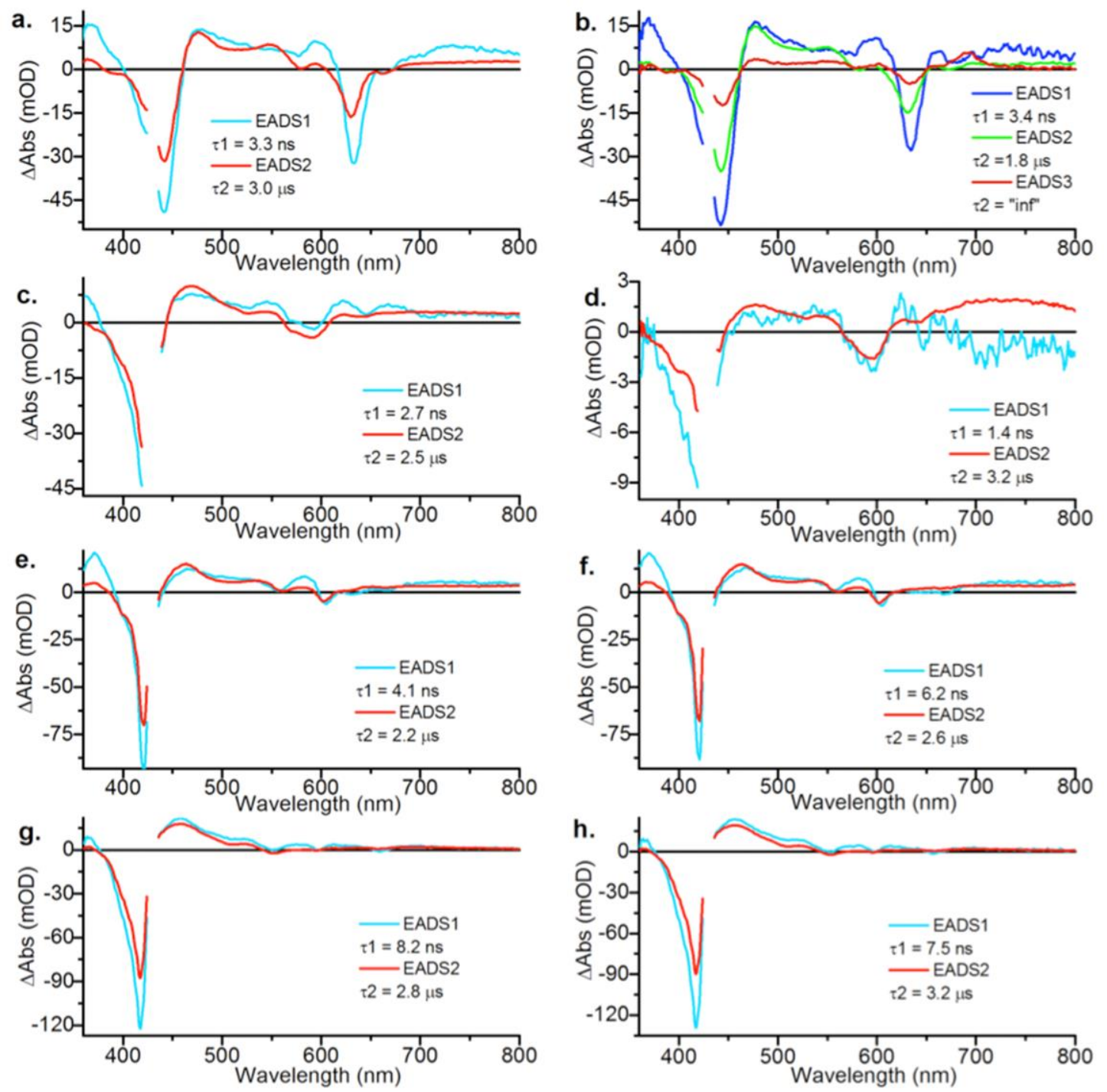

Figure 8. Evolution Associated Difference Spectra (EADS) resulting from global analysis using a sequential model of visible transient absorption data collected between $0.6 \mathrm{~ns}$ and $2.7 \mu \mathrm{s}$. EADS are shown for Pchlide (a), POR with NADPH and Pchlide (b), protopheophorbide (c), POR with NADPH and protopheophorbide (d), C13C15 methylester Pchlide (e), POR with NADPH and C13C15 methylester pchlide (f), C13OH Pchlide (g), POR with NADPH and $\mathrm{C} 13 \mathrm{OH}$ Pchlide (h). All data could be fitted using 2 EADS, except for POR with NADPH and Pchlide, which required 3 EADS due to the formation of the hydride transfer intermediate (see spectrum in red in b). The absence of any additional intermediates for the Pchlide analogues in the presence of POR implies impaired photochemistry. 
a

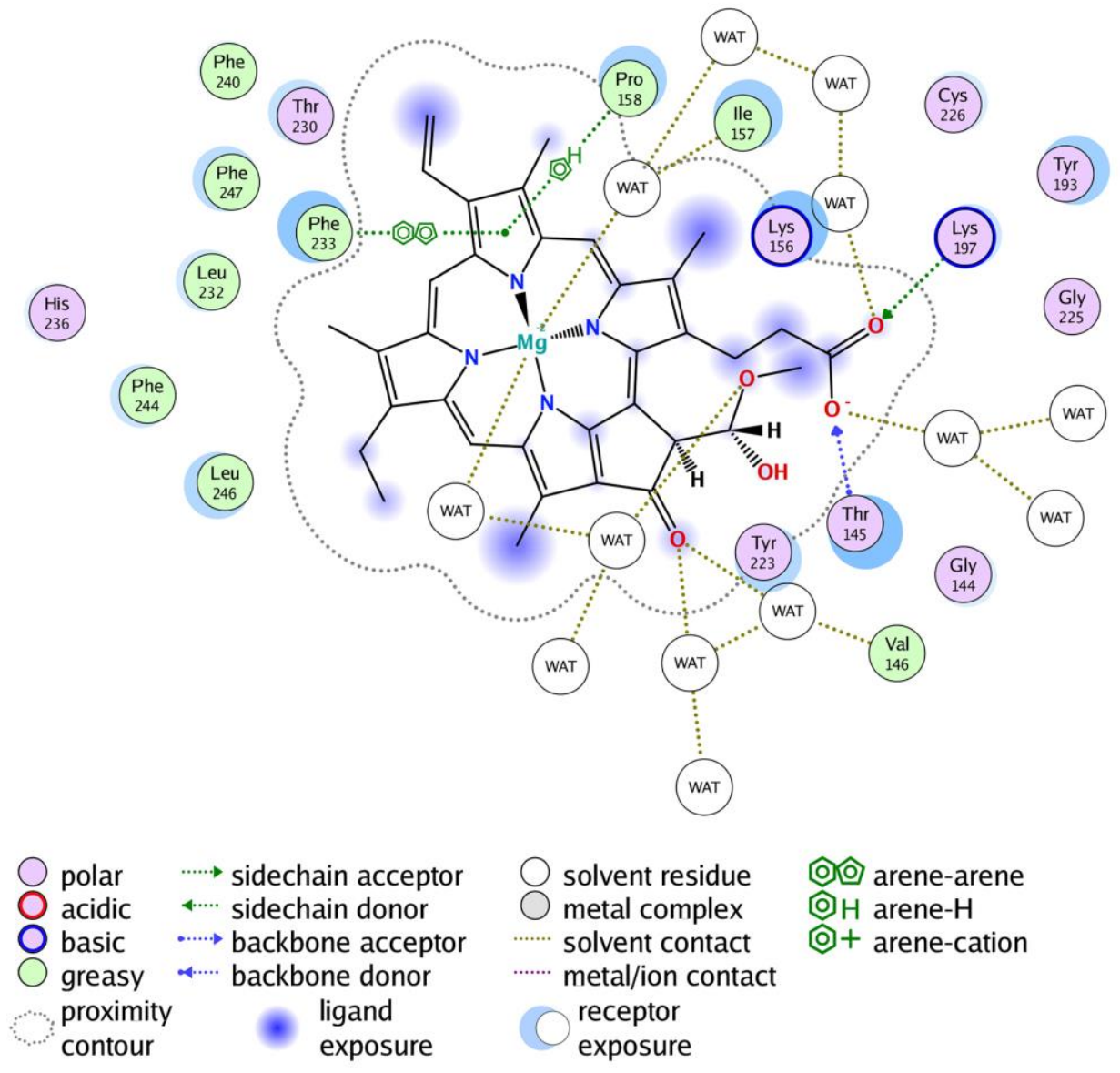

b

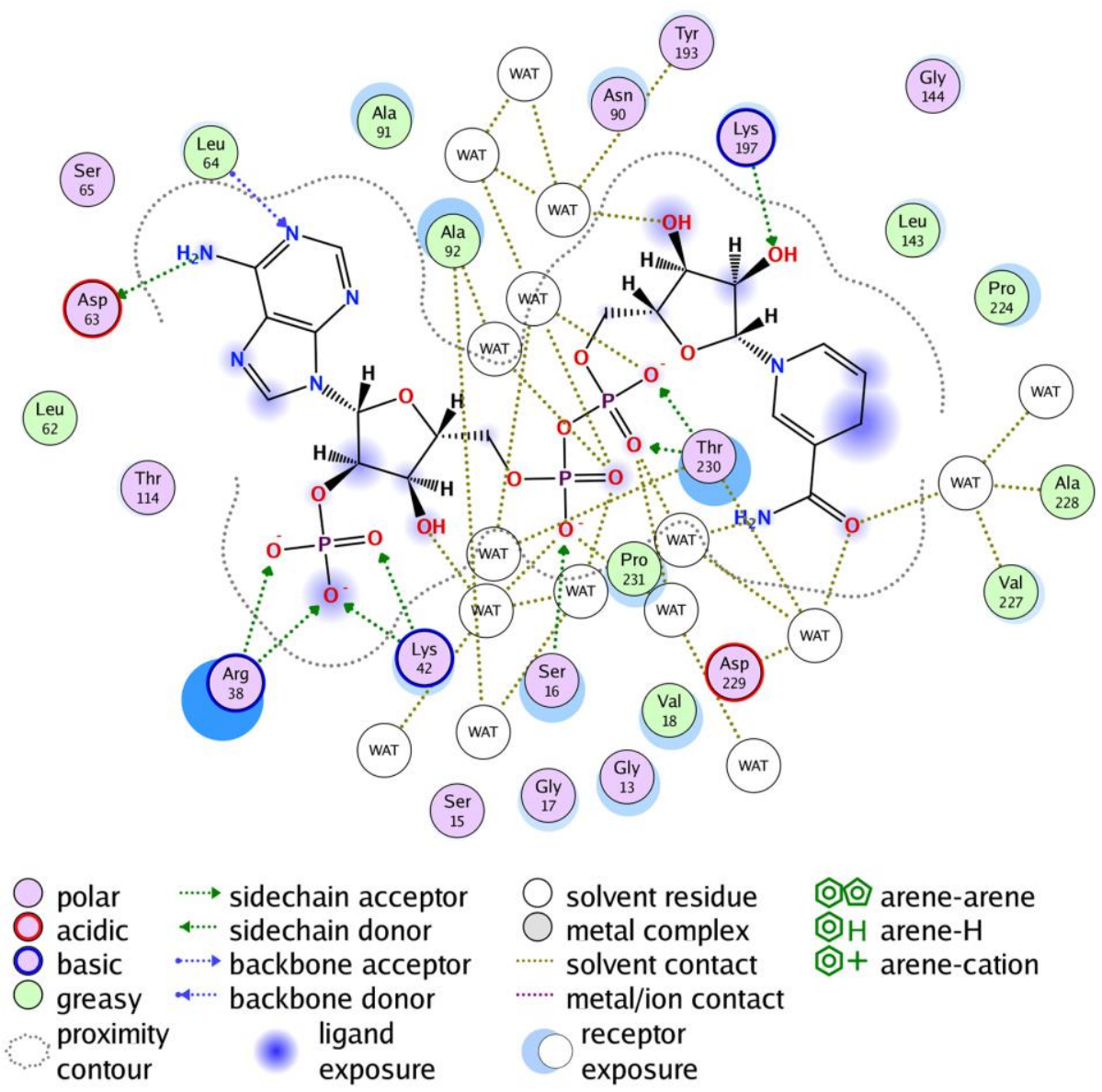


Figure 9. Potential interactions in T. elongatus POR ternary complex model. (a) The Pchlide molecule was chosen as the ligand, POR protein and solvent were chosen as the receptor. The 2D interaction map was calculated through Molecular Operating Environment software (Chemical Computing Group). (b) The NADPH molecule was chosen as the ligand, POR protein and solvent were chosen as the receptor. The 2D interaction map was calculated through Molecular Operating Environment software (Chemical Computing Group). 


\section{Extended data tables}

\begin{tabular}{|c|c|c|c|}
\hline & TePOR* & TePOR-NADPH & SsPOR-NADPH \\
\hline \multicolumn{4}{|l|}{ Data collection } \\
\hline Space group & P $3{ }_{1} 21$ & P $3{ }_{1} 21$ & $\mathrm{P} 22_{1} 22_{1}$ \\
\hline \multicolumn{4}{|l|}{ Cell dimensions } \\
\hline$a, b, c(\AA)$ & 66.8566 .85133 .01 & 66.9966 .99131 .97 & 56.9672 .89155 .99 \\
\hline$\alpha, \beta, \gamma\left({ }^{\circ}\right)$ & 9090120 & 9090120 & 909090 \\
\hline \multirow[t]{2}{*}{ Resolution $(\AA)$} & $53.08-1.27$ & $43.57-2.1$ & $28.57-1.87$ \\
\hline & $(1.315-1.27)^{* *}$ & $(2.175-2.1)$ & $(1.91-1.87)$ \\
\hline$R_{\text {merge }}$ & $0.0928(0.8745)$ & $0.1092(0.7126)$ & $0.097(0.881)$ \\
\hline$I / \sigma I$ & $14.73(2.36)$ & $12.60(3.18)$ & $11.80(2.1)$ \\
\hline Completeness (\%) & $98.76(96.46)$ & $99.96(100.00)$ & $94.90(86.50)$ \\
\hline Redundancy & $17.5(12.1)$ & $9.2(8.9)$ & $5.6(6.0)$ \\
\hline \multicolumn{4}{|l|}{ Refinement } \\
\hline \multirow[t]{2}{*}{ Resolution $(\AA)$} & $53.08-1.27$ & $43.57-2.1$ & $28.57-1.87$ \\
\hline & $(1.315-1.27)$ & $(2.175-2.1)$ & $(1.91-1.87)$ \\
\hline No. reflections & $91463(9068)$ & 20674 (2023) & $51836(3223)$ \\
\hline$R_{\text {work }} / R_{\text {free }}$ & $0.1526 / 0.1858$ & 0.1672 / 0.1997 & $0.170 / 0.179$ \\
\hline \multicolumn{4}{|l|}{ No. atoms } \\
\hline Protein & 2309 & 2224 & 4761 \\
\hline Ligand/ion & 2 & 48 & 97 \\
\hline Water & 363 & 158 & 416 \\
\hline \multicolumn{4}{|l|}{$B$-factors $\left(\AA^{2}\right)$} \\
\hline Protein & 19.09 & 37.01 & 23.2 \\
\hline Ligand/ion & 21.17 & 50.22 & 15.2 \\
\hline Water & 34.55 & 41.16 & 32.4 \\
\hline \multicolumn{4}{|l|}{ R.m.s. deviations } \\
\hline Bond lengths $(\AA)$ & 0.010 & 0.007 & 0.019 \\
\hline Bond angles $\left({ }^{\circ}\right)$ & 1.17 & 0.7 & 1.472 \\
\hline
\end{tabular}

\begin{tabular}{lllclc}
\hline \multirow{2}{*}{$\begin{array}{c}\text { Pchlide and its analouge } \\
\text { or } T e \text { POR variants }\end{array}$} & \multicolumn{1}{c}{$k_{\text {cat }}$} & $K_{\mathrm{m}} / K_{\mathrm{i}} \mathrm{Pchlide}^{1}$ & $K_{\mathrm{d}}$ Pchlide & $k_{\text {hydride }}$ & $k_{\text {proton }}$ \\
\cline { 2 - 6 } & $0.023 \pm 0.001$ & $3.6 \pm 0.4$ & $1.9 \pm 0.7$ & $2.36 \pm 0.11$ & $2.23 \pm 0.05$ \\
\hline Pchlide (I) & $\mathrm{NA}$ & $8.5 \pm 0.3$ & $9.7 \pm 3.5$ & $\mathrm{NA}$ & $\mathrm{NA}$ \\
Protopheophorbide (II) & $\mathrm{NA}$ & $\mathrm{NA}$ & $\mathrm{NA}$ & $\mathrm{NA}$ & $\mathrm{NA}$ \\
C17 methylester Pchlide (III) & $\mathrm{NA}$ & $9.7 \pm 1.1$ & $6.1 \pm 1.2$ & $\mathrm{NA}$ & $\mathrm{NA}$ \\
C13OH Pchlide (IV) & $\mathrm{NA}$ & $50.8 \pm 3.6$ & $46.7 \pm 4.6$ & $\mathrm{NA}$ & $\mathrm{NA}$ \\
C13C15 methylester Pchlide (V) & $\mathrm{NA}$ & $\left(\mu 10^{6} \mathrm{~s}^{-1}\right)$ & $\left(\times 10^{4} \mathrm{~s}^{-1}\right)$ \\
K156A & $0.017 \pm 0.001$ & $3.11 \pm 0.55$ & $\mathrm{NA}$ & $2.40 \pm 0.03$ & $2.26 \pm 0.14$ \\
Y223A & $0.020 \pm 0.004$ & $33.2 \pm 10.2$ & $\mathrm{NA}$ & $1.77 \pm 0.42$ & $2.39 \pm 0.46$ \\
Y223F & $0.017 \pm 0.001$ & $11.6 \pm 1.98$ & $\mathrm{NA}$ & $1.80 \pm 0.15$ & $0.93 \pm 0.07$ \\
\hline
\end{tabular}


Table 1. Data collection and kinetics parameters of POR

(a) Data collection and refinement statistics (molecular replacement). *A single crystal was used for each data collection. **Values in parentheses are for highestresolution shell. (b) Kinetics parameters of T. elongatus POR substrate analogues and variants. ${ }^{1} K_{\mathrm{i}}$ values were measured for non-active substrate analogues. NA: not applicable for non-active substrate analogues. The values shown in the table are mean \pm standard error, $n=3$ for steady state kinetics measurements, $n=5$ for hydride and proton transfer measurements. 


\section{Structural basis for enzymatic photocatalysis in chlorophyll biosynthesis}

Shaowei Zhang* ${ }^{1}$, Derren J. Heyes* ${ }^{1}$, Lingling Feng $*^{3}$, Wenli Sun $*^{2}$, Linus O. Johannissen*1, Huanting Liu ${ }^{6}$, Colin W. Levy ${ }^{1}$, Xuemei Li ${ }^{4}$, Ji Yang ${ }^{6}$, Xiaolan Yu ${ }^{6}$, Min Lin², Samantha J. O. Hardman ${ }^{1}$, Robin Hoeven ${ }^{1}$, Michiyo Sakuma ${ }^{1}$, Sam Hay ${ }^{1}$, David Leys ${ }^{1}$, Zihe Rao ${ }^{4}$, Aiwu Zhou $^{\ddagger 3}$, Qi Cheng ${ }^{\ddagger 2,5,6}$ and Nigel S. Scrutton ${ }^{\ddagger 1}$

${ }^{1}$ Manchester Institute of Biotechnology, The University of Manchester, 131 Princess Street, Manchester M1 7DN, UK

${ }^{2}$ Biotechnology Research Institute, Chinese Academy of Agricultural Sciences, Beijing 100081, China

${ }^{3}$ Department of Pathophysiology, Key Laboratory of Cell Differentiation and Apoptosis of the Chinese Ministry of Education, Shanghai Jiao Tong University School of Medicine, 280 South Chongqing Rd., Shanghai 200025, China

${ }^{4}$ National Laboratory of Biomacromolecules, Institute of Biophysics, Chinese Academy of Sciences, Beijing 100101, China

${ }^{5}$ College of Biotechnology and Bioengineering, Zhejiang University of Technology, Hangzhou 310014, China

${ }^{6}$ C4-101, Nitrogen Fixation Laboratory, Qi Institute, 555 Chuangye Road, Dayun Town, Jiashan County, Jiaxing 314000, Zhejiang Province, China

*These authors contributed equally to this work.

${ }^{\ddagger}$ Correspondence can be addressed to: nigel.scrutton@manchester.ac.uk; chengqi@,caas.cn; awz20@shsmu.edu.cn 


\section{Supporting information}

\section{Content}

\section{Supplementary Methods}

\section{Supplementary Figures}

\section{Supplementary Tables}

\section{MD Parameters for NADPH and PChlide}

\section{Supplementary Methods}

\section{Sample preparation and site directed mutagenesis}

All chemicals were obtained from Sigma-Aldrich. The POR gene from Thermosynechococcus elongatus was codon optimized for overexpression in E. coli, synthesized (GeneArt, ThermoFisher) and subcloned into pET21a with a C-terminal 6-His tag. The Synechocystis PC6803 POR gene was cloned into the pE-SUMO3 expression vector. ${ }^{27}$ Site-directed mutagenesis of the por gene was performed using the QuikChange kit (Stratagene) to mutate residues. The primers that were used (MWG Eurofins, Germany) are shown in the supplementary information (Table S1). The correct mutations were confirmed by DNA sequencing (MWG Eurofins, Germany). The recombinant plasmids were transformed into $E$. coli BL21 (DE3) and protein was expressed by growing transformed cells in LB containing $100 \mu \mathrm{g} / \mathrm{mL}$ ampicillin at $25^{\circ} \mathrm{C}$ until $\mathrm{OD}_{600}$ reached $0.6-0.8$ followed by induction with $0.3 \mathrm{mM}$ isopropyl- $\beta$-D-thiogalactopyranoside overnight. Cells were harvested by centrifugation at 6 $\mathrm{krpm}$ for 10 minutes at $4^{\circ} \mathrm{C}$, resuspended in lysis buffer (20 mM HEPES pH 7.5, $500 \mathrm{mM}$ $\mathrm{NaCl}, 25 \mathrm{mM}$ imidazole, $1 \mathrm{mM}$ DTT for T. elongatus POR or $20 \mathrm{mM}$ Tris-HCl, pH 7.4, 300 $\mathrm{mM} \mathrm{NaCl}$, and 20mM imidazole for Synechocystis POR) and lysed by sonication (15 x $15 \mathrm{~s}$ ). After centrifugation at $20,000 \mathrm{rpm}$ for 1 hour at $4{ }^{\circ} \mathrm{C}$ the supernatant was loaded onto a $5-\mathrm{ml}$ HiTrap FF crude column and eluted by a $0.02-0.3 \mathrm{M}$ imidazole gradient. The Synechocystis POR was treated with protease SENP2 to remove the SUMO tag, dialyzed into $20 \mathrm{mM}$ Tris$\mathrm{HCl} \mathrm{pH} 7.4,0.05 \mathrm{M} \mathrm{NaCl}$, loaded onto a 5-ml Hitrap Q HP column and eluted by a $\mathrm{NaCl}$ gradient $(0.05-0.5 \mathrm{M})$. Both proteins were further purified by a Superdex 75 gel filtration column and pure protein fractions were collected and concentrated for crystallization. 


\section{Crystallization, data collection, and structure determination}

Synechocystis POR was concentrated to $26 \mathrm{mg} / \mathrm{ml}$ in $20 \mathrm{mM}$ Tris- $\mathrm{HCl} \mathrm{pH} 7.4,150 \mathrm{mM} \mathrm{NaCl}$ and T. elongatus POR was concentrated to 10 to $15 \mathrm{mg} / \mathrm{mL}$ in $20 \mathrm{mM}$ HEPES pH 7.5, $150 \mathrm{mM}$ $\mathrm{NaCl}$ and $1 \mathrm{mM}$ DTT. Crystallization was performed using the sitting drop vapor diffusion technique (200 $\mu \mathrm{L}$ crystallization reagent mixed with $180 \mu \mathrm{L}$ protein and $20 \mu \mathrm{L}$ seed stock). For T. elongatus POR, crystals were obtained from $0.01 \mathrm{M}$ zinc chloride, $0.1 \mathrm{M}$ sodium acetate $\mathrm{pH}$ 5.0, 20 \% w/v PEG 6K (A12, PACT screen, Molecular Dimensions Ltd., Newmarket, UK) after incubation at $4^{\circ} \mathrm{C}$ over a period of $\sim 72 \mathrm{~h}$. For Synechocystis POR crystals were obtained with $20 \%$ PEG3350 in $0.2 \mathrm{M}$ tri-lithium citrate, $0.01 \mathrm{M}$ zinc chloride after incubation at $20^{\circ} \mathrm{C}$ for approximately 10 days. Although coenzyme-bound POR crystals were obtained by soaking (T. elongatus) or co-crystallization (Synechocystis) with NADPH, it should be noted that this form of the coenzyme is unstable and it is likely that crystals contained a mixture of NADPH or $\mathrm{NADP}^{+}$as the bound ligand. It is impossible to distinguish which form of the coenzyme is present in the crystals as similar R factor and $\mathrm{R}$ free values are obtained for each $\left(\mathrm{NADP}^{+}\right.$: $\mathrm{R}=0.1550, \mathrm{R}$ free $=0.1930$, NADPH: $\mathrm{R}=0.1549$, $\mathrm{R}$ free $=0.1930$ ). However, lower resolution data obtained from crystals of $T$. elongatus POR soaked for 5 minutes under strictly anaerobic conditions with NADPH (thus avoiding formation of $\mathrm{NADP}^{+}$) reveal the coenzyme binds in a similar conformation (Extended data Figure 4). Crystals were cryo protected with the addition of $20 \%$ PEG 200 to the reservoir solution prior to flash cooling in liquid nitrogen. Individual datasets were collected from single cryo protected crystals at beamlines i03, i04 and i04-1 (Diamond Light Source) for T. elongatus POR and at SSRF BL17U beamline for Synechocystis POR. All data were indexed, scaled and subsequently integrated with Xia2. Structure determination was initially performed by molecular replacement in Phaser using a search model derived from the previously solved oxidoreductase structure from Mycobacterium paratuberculosis (3RD5). A combination of automated and manual rebuilding and refinement in Phenix and COOT were used to produce each of the refined models. Validation with both Molprobity and PDB_REDO were integrated into the iterative rebuild process. Complete data collection and refinement statistics are available in Extended data Table 1b. The atomic coordinates and experimental data have been deposited in the Protein Data Bank (www.pdb.org). All figures were made using PyMOL software. It was not possible to obtain crystals of the ternary complex, either by co-crystallization or by soaking. To prepare the ternary complex, the purified POR protein was incubated overnight with equal molar concentration of Pchlide and excess NADPH (more than 10 fold) at room temperature. The sample was concentrated and loaded onto a gel filtration column (Superdex 200) in $20 \mathrm{mM}$ 
HEPES buffer with $150 \mathrm{mM} \mathrm{NaCl}$ and $1 \mathrm{mM}$ DTT. Binding of the Pchlide substrate causes oligomerization of the POR protein (Figure S2), resulting in an inhomogeneous mixture of oligomeric states of protein.

\section{Synthesis of Pchlide and Pchlide analogues}

The Pchlide substrate was produced and purified Rhodobacter capsulatus ZY5 cultures. ${ }^{28}$ All Pchlide analogues were synthesized from pheophorbide $a$ and analyzed by NMR and mass spectrometry analyses. ${ }^{11}$

\section{Steady-state activity and substrate binding measurements.}

The concentrations of the different pigments were determined using the following extinction coefficients in aqueous solution: NADPH, $6.22 \mathrm{mM}^{-1} \mathrm{~cm}^{-1}$ at $340 \mathrm{~nm}$; Pchlide, $24.95 \mathrm{mM}^{-1} \mathrm{~cm}^{-}$ ${ }^{1}$ at $630 \mathrm{~nm}$; and Chlide, $69.95 \mathrm{mM}^{-1} \mathrm{~cm}^{-1}$ at $670 \mathrm{~nm}$. Steady-state activity measurements were carried out at $40^{\circ} \mathrm{C}$ using a Cary 50 spectrophotometer (Agilent Technologies) to measure the initial rates of Chlide production over a range of Pchlide concentrations of $0.1 \mu \mathrm{M}$ POR and $100 \mu \mathrm{M}$ NADPH in $0.1 \%$ Triton X-100, $0.1 \%$ (v/v) 2-mercaptoethanol, $50 \mathrm{mM}$ Tris/HCl, $\mathrm{pH}$ 7.5. A $625 \mathrm{~nm}$ high power LED (Thorlabs Inc.) provided continuous illumination (100 $\mu$ mole $\mathrm{m}^{-2} \mathrm{~s}^{-1}$ ) during the reaction assay. The $K_{\mathrm{m}}$ and $V_{\max }$ values were obtained by fitting the initial rates of Chlide synthesis against the concentration of Pchlide to the following equation:

$$
v=\frac{V_{\max }[\text { Pchlide }]}{K_{m}+[\text { Pchlide }]}
$$

Activity assays to measure the apparent inhibition constants $\left(K_{\mathrm{i}}^{\mathrm{app}}\right)$ for Pchlide analogues were performed using $0.1 \mu \mathrm{M}$ POR, $10 \mu \mathrm{M}$ Pchlide and $100 \mu \mathrm{M}$ NADPH over a range of Pchlide analogue concentrations. The $K_{\dot{\mathrm{i}}}^{\text {app }}$ values for the analogues were obtained by plotting the percentage inhibition versus the analogue concentration and fitting to the Morrison equation:

$$
\frac{v_{i}}{v_{0}}=1-\frac{\left([\mathrm{E}]+[\mathrm{I}]+K_{i}^{a p p}\right)-\sqrt{\left([\mathrm{E}]+[\mathrm{I}]+K_{i}^{a p p}\right)^{2}-4[\mathrm{E}][\mathrm{I}]}}{2[\mathrm{E}]}
$$

The binding of Pchlide was measured by following the red-shift in absorbance at $642 \mathrm{~nm}$ and the binding of the Pchlide analogues was monitored by measuring changes in fluorescence emission spectra at increasing POR concentrations in a Cary Eclipse fluorimeter (Agilent 
Technologies), using an excitation wavelength of $430 \mathrm{~nm}$ and slit widths of $5 \mathrm{~nm}$. The apparent $K_{\mathrm{d}}$ values were obtained by fitting the absorbance or fluorescence changes ( $\Delta$ spec) against the concentration of POR using the following equation:

$$
\Delta \mathrm{spec}=\frac{\Delta \mathrm{spec}_{\max }[\mathrm{POR}]}{K_{d}+[\mathrm{POR}]}+\mathrm{c}
$$

All data were fitted using Origin 9.0 Software (OriginLab, Northampton, MA).

\section{Laser flash photolysis}

Absorption transients at $696 \mathrm{~nm}$ were measured in a $1 \mathrm{~cm}$ pathlength cuvette at $298 \mathrm{~K}$ using an LKS-60 flash photolysis instrument (Applied Photophysics Ltd). ${ }^{14}$ Dark assembled enzyme-NADPH-Pchlide ternary complex samples were excited with a $6 \mathrm{~ns}$ laser pulse at 532 $\mathrm{nm}(\sim 180 \mathrm{~mJ})$ using the second harmonic of a Q-switched Nd-YAG laser (Brilliant B, Quantel). The reaction samples were prepared by taking $1 \mu \mathrm{M}$ Pchlide and $250 \mu \mathrm{M}$ NADPH in the presence of $50 \mu \mathrm{M}$ enzyme for wild-type POR and $100 \mu \mathrm{M}$ enzyme for the $\mathrm{Y} 223 \mathrm{~F}$ and Y223A variants. Rate constants were measured from the average of at least five time dependent absorption measurements by fitting to a single exponential function.

\section{Ultrafast pump-probe transient absorption spectroscopy.}

Measurements were carried out as described previously ${ }^{19}$ using an excitation wavelength of $450 \mathrm{~nm}$ with $0.5 \mu \mathrm{J}$ pulse power and a beam diameter of $\sim 150 \mu \mathrm{m}$. Samples $(1.5 \mathrm{ml})$ were flowed at a rate of approximately $30 \mathrm{ml} / \mathrm{min}$ through a $0.2 \mathrm{~mm}$ pathlength quartz cell (at room temperature) to ensure that a different area of the sample is excited with each pump laser pulse. Samples were measured upon for $<5$ minutes to prevent any signals arising from any Chlide product in the reaction mixture. Samples were prepared in the dark containing $500 \mu \mathrm{M}$ POR, $200 \mu \mathrm{M}$ Pchlide (or Pchlide analogue) and 4 mM NADPH in $50 \mathrm{mM}$ Tris-HCl, $\mathrm{pH} 7.5$ with $0.1 \% 2$-mercaptoethanol, and $0.5 \%$ Triton X-100. Data were collected for approximately 30 mins per dataset at random time delays ranging between 300 fs and $2 \mu \mathrm{s}$ and the datasets were analyzed globally using the open-source software Glotaran. ${ }^{29}$ 


\section{Computational methods}

1. Building a full-length ternary structure of T. elongatus POR. A full-length model of $T$. elongatus POR was built from the NADPH-bound T. elongatus crystal structure with the missing loops taken from the NADPH-bound Synechocystis crystal structure, with necessary mutations (Figure S1) performed using Scwrl4. ${ }^{30}$

2. Molecular Docking. Initial structures of Pchlide-bound T. elongatus POR were generated by docking using AutoDock Vina. ${ }^{31}$ Loop 1 and helix 1 were not included as these are very flexible regions whose precise conformations will affect the conformation of Pchlide. The search volume was a cube with $60 \AA$ sides with the nicotinamide nitrogen of NADPH as the centre and an exhaustiveness of 75 was used to generate 20 conformations. The following residues were flexible during the docking: Y94, T145, K194 and Y223. Crystallographic waters were not included in the docking process. The highest-scoring structure that is consistent with the stereochemistry was selected for further modelling. Loop 1 and helix 1 were rebuilt before further modelling.

3. Molecular Dynamics (MD) simulations parameters. All MD simulations were carried out in Gromacs 5.0.4 ${ }^{32}$ using the Amber14 force field ${ }^{33}$ with the system in a solvation box of at least $12 \AA$ with counter-ions generated in AmberTools, retaining crystallographic waters, totalling 48,561 atoms for the ternary complex and 48,483 and 48,416 atoms for the NADPHbound and apo structures, respectively. The following parameters were used: constant pressure (1 bar), $10 \AA$ van der Waals and electrostatic cutoffs, particle mesh Ewald for longrange electrostatics, LINCS bond restraints and periodic boundary conditions and a 2 fs time step. Bonding parameters for Pchlide and NADPH were generated using the AmberTools ANTECHAMBER module ${ }^{34}$ and charges were parameterized by RESP fitting to a $\mathrm{HF}^{35} / 6$ $31 \mathrm{G}^{*}$ structures optimized in Gaussian09 Revision D.01. ${ }^{36}$ Initial parameters were generated for protopheophorbide, and $\mathrm{Mg}^{2+}$ parameters were added by fitting to Gaussian09 distance and angle scans in a $\mathrm{B}^{2} \mathrm{LYP}^{37}$ model with the LANL2DZ ${ }^{38}$ basis set applied to the Mg and 6$31 G^{*}$ to the other atoms (Figures S12 and S13). The NADPH and Pchlide parameters used are listed in Gromacs format in the supporting information. Unrestrained MD simulations of all three forms of the enzyme were performed to confirm the stability of the final ternary complex model and compare the relative structural flexibilities of the NADPH-bound and apo structures. These simulations were run for $650 \mathrm{~ns}$ to ensure that convergence of the RMSD plots (Extended data Figure 3). 
4. Simulated Annealing. The candidate ternary structures were relaxed by simulated annealing, with $20 \times 1 \mathrm{~ns}$ heating and cooling cycles applied as follows (for a total of $20 \mathrm{~ns}$ ): 500 ps at $300 \mathrm{~K}, 100$ ps heating to $340 \mathrm{~K}, 300$ ps at $340 \mathrm{~K}$ and 100 ps cooling to $300 \mathrm{~K}$. No constraints were applied to the protein, but the following distance restraints were applied to maintain the Pchlide in a bound conformation relative to the protein and NADPH, with a force constant of $10 \mathrm{~kJ} \mathrm{~mol}^{-1} \AA^{-2}$ : (i) between Pchlide C17 and NADPH C1 (the proposed hydride donor, $r_{0}=2 \AA$ ), (ii) between Pchlide C18 and Y193 hydroxyl proton (the proposed proton donor, $r_{0}=2 \AA$ ); (iii) between Pchlide C17 and L143 C $\alpha$ (at the base of the active site pocket, $r_{0}=9 \AA$ ). The structure after $20 \mathrm{~ns}$ of simulation was chosen for further modelling (Extended data Figure 5).

5. Umbrella sampling. The potential of mean force (PMF) for Pchlide binding was calculated as a function of the distance between the Pchlide $\mathrm{Mg}^{2+}$ and the base of the active site pocket, defined as the C $\alpha$ of residues N90, L143 and L222. Sampling was carried out in bins no more than $0.7 \AA$ apart (Extended data Figure 5) with a force constant of $25 \mathrm{~kJ} \mathrm{~mol}^{-1} \AA^{-2}$, for a total of $50 \mathrm{~ns}$ per bin. A representative structure was chosen from the structures within the range $14.5 \AA<\mathrm{R}<14.7 \AA$. For these structures the RMSD was calculated for heavy atoms of the Pchlide, NADPH nicotinamide group and sidechains of first-shell residues around the Pchlide (residues 94, 144, 145, 146, 152, 153, 156, 157, 158, 193, 194, 197, 223, 224, 225, 226, 227 , $228,230,232,233,236,240,244,246,247)$, and the structure with the smallest RMSD relative to the average structure was selected.

\section{Additional Methods References}

1. Wei, Z., Yan, Y., Carrell, R. W. \& Zhou, A. Crystal structure of protein Z-dependent inhibitor complex shows how protein $\mathrm{Z}$ functions as a cofactor in the membrane inhibition of factor X. Blood 114, 3662-3667 (2009).

2. Heyes, D. J., Ruban, A. V., \& Hunter, C. N. Protochlorophyllide oxidoreductase: spectroscopic characterization of the dark reactions. Biochemistry 42, 523-528 (2003).

3. Snellenburg, J. J., Laptenok, S. P., Seger, R., Mullen, K. M. \& Stokkun, I. H. M. Glotaran: A Java-Based Graphical User Interface for the R Package TIMP. J. Stat. Softw., 49, 1-22 (2012). 
4. Krivov, G.G., Shapovalov, M.V. \& Dunbrack Jr., R.L. Improved prediction of protein side-chain conformations with SCWRL4. Proteins: Struct., Funct., Bioinf. 77, 778-795 (2009).

5. Trott, O. \& Olson, A.J, AutoDock Vina: improving the speed and accuracy of docking with a new scoring function, efficient optimization, and multithreading, J. Comp. Chem. 31, 455-461 (2009)

6. Hess, B., Kutzner, C., Spoel, D. van der \& Lindahl, E. Gromacs 4: algorithms for highly efficient, load-balanced, and scalable molecular simulation. J. Chem. Theory Comp. 4, 435-447 (2008).

7. Maier, J.A. et al ff14SB: Improving the accuracy of protein side chain and backbone parameters from ff99SB. J. Chem. Theory Comp. 11, 3696-3713 (2015).

8. Wang, J., Wang, W., Kollman P. A. \& Case, D. A. Automatic atom type and bond type perception in molecular mechanical calculations. J. Mol. Graphics Model., 25, 247260 (2006)

9. Roothaan, C.C.J. New Developments in Molecular Orbital Theory, Rev. Mod. Phys., 23, 69-89 (1951).

10. Frisch, M. et al Gaussian 09 D. 01, Gaussian. Inc., Wallingford CT (2016).

11. Becke, A. D., Density-functional thermochemistry. III. The role of exact exchange. $J$. Chem. Phys. 98, 5648-5652 (1993).

12. Wadt, W.R., Hay, P.J. Ab initio effective core potentials for molecular calculations potentials for main group elements Na to Bi. J. Chem. Phys., 82, 284-298 (1985).

13. Ashkenazy H. et al ConSurf 2016: an improved methodology to estimate and visualize evolutionary conservation in macromolecules. Nucl. Acids Res.44, W344-W350 (2016). 


\section{Supplementary Figures:}

Unconserved 12345678910 Conserved

GxxxGxG motif

Thermosynechoco M SDQPRPTVI

Limnoraphis robMEOHOXSTV

Synechocystis PMK----PTV

Anabaena_variabMA QDRKSTV

Crinalium_epipsMAQDLKSTV

Calothrix paras MSONSKSTV

Lyngbya_majuscuMENDHKSTV

Synechococcus_sMAEQYTPTV

Stanieria_cyanoMS Y - -QKTV

Microcystis_aerMVQDQKPTV

Oscillatoriales MTODOKPTV

Fischerella_sp_MEHNRKSTV

Cyanothece_sp_MEQQRKSTA

Nostoc_punctifoMVQDRKSTV

Nicotiana_tabac KKT LRKGNV

zea_mays KKTLRKGTA

Marchantia_paleKKTATKSTC

Pinus_mugo KKTDRKGNV

Physcomitrella_KKTDTKSTV

Chlamydomonas IPATQOKOTA

Daucus_carota KKTLRKGSV

Cyanidioschyzon-GPKQSQIA

Prochlorococcus MS KD I K GI. A

Consistency

6543375775 Thermosynechoco . . . . 60

$60 \ldots \ldots \ldots 70$

$70 \ldots \ldots 8$

$30 \ldots \ldots \ldots 40 \ldots \ldots$ YATKALANR- G HUIMACFN LEKAEQAAKN QAANALAKK- GWYVVMACRN VEKTEKAAQS YGRKALIDK- GWHVIMACRN LDKTOKVADE YAAKALAKR- GWHVVMACRN LEKAEQAAQE YGALALAKR- GWHVVMGCRD LDKAQKAAET YAAKAAAKK- GWHVVMACRN LEKTORVANE BAARALATTW GWHVMACRN LDKAEKAAQE QAARALAEK- GHHVMACRD LSKTETVAKS YAAKSLANR- GWHVVMACRN LEKAKQAAKE YAAKSLAQR- GMFVVMACRD IPKMEQAAKE YAAKAFVDR- GWFVVMACRD IPKTEQAVAS YSAKALAKR- GWHVVMACRD LAKAEKAAQT QAAKALADL- GWYVVMACRD IEKTKRVARE YAAKALAER- GWYVVMACRD VAKAQLAAQS ATAKAIGETG BWHVIMACRD ELKAEKAAKS ATAKALAETG KWHVIMACRD FLKASRAAKA ATAKALADTG ENHVIMACRD FLKAERAARS ATAKALGESG KWHIIMACRD FLKAERMARS ATAKVLADSG EWHVIMACRD FLKAERAAKS NAAKALAATG BHHVVMACRD ELKAEQAAKK ATAKALAETG KNHVIMACBD FLKAERAAKS YAARALVNKG NMHIVMACRD IDRAERAADS NTLKPLLRE- GWEVIAVNRS NRRAIKIAEE ILHLLSSI ASVRGFVESF RALNR--PLR ALVICNARVYY Limnoraphis rObVGIPA-D-SY TIMPIDIASI ESVROEVKTF RESGK--SID AIVTCHAMVI Synechocystis_PLGFPK-D-SY TIIKLDLGYL DSVRRFVAQF RELGR--PLK ALVCNAAVYF Anabaena_variabVGIPK-D-SY TIIBIDIGSL DSVRQFVNDF RATGK--SLD ALICNAAIYM Crinalium_epipsVGIPQ-G-SY TLIBIDLGSL DSVRRFVDNF RARGI--SLD ALVINAAIYM Calothrix parasVGMSP-D-SY TIMHLDLASL KSVRQFVNTF RESGK--SLD ALVENANVYI Lyngbya_majuscuVGIPK-D-SY TAIYIDLASF ASVROFVNAF RATGR--TLD AIVICNANIYI Synechococcus_SVGMKP-E-NY TILPIDIADL SKVRLFVERE RATGK--PID ALVICNAAVYI Stanieria_cyanoVRIPE-G-NY SIIKLDLASL ASVRQFVEDF RATGR--YLD ALVCNAAVYL Microcystis_aerLNIPR-D-NY CIEFIDIGSI NSVRRFVKNF RALGR--SIT ALVICNAAIYI OscillatorialesLGIPK-D-SY TILHIDLASL QSVREFVKDF RATGR--SLN ALVCNAAIYM Fischerella_sP_VGIPQ-D-SY TIMHIDISSI ESVRQFVKDF RASGK--SLE ALVCNAAIYM Cyanothece_sp_ VGMPA-D-SY NIIYIDIASI QSVREFVKYF RALSR--PID ALVCNAAIYM Nostoc_punctifoVGIPH-QGSY TIMHIDIGSI DSVRQFVKNF RASGH--SLD ALVCNAAIYM Nicotiana_tabacVGIPK-E-NY TVMHLDIASI, BSVROEVDTP RRSGR--PLD ALVICNA2VYI Marchantia_paleVGIPK-D-SY TVIBCDIASF DSVRAFVDNF RRTER--QLD VLVCNAAVYF PInUs_mugo VGIPK-E-NY SVMBLDIASL ESVRQFADNF RRSGR--PID VLVCNAAIYL Physcomitrella_VGMPK-E-NY TVMHCDLSSL NSVRQFVDNF RRSGR--PLD VLVCNAAVYL Chlamydomonas_rVGMPA-G-SY SILHLDISSL ESVROEVONF KASGR--RID ALVICNANVYL Daucus_carota AGMPK-E-NY TIMHLDIASL DSVRQEVETF RRSER--PLD VLVCNAAVYF CyanidioschyzonVNLPK-D-AY TVLBCDIADF ASVIKFVREL SSVARVDHLD ALIONAATWH Prochlorococcus SLTKE VVRN HFIEIDISNL DDVRKGCDQI LERENKEPIN SLICNAAVYK Consistency $\quad 777750607978767$

\begin{tabular}{|c|c|c|c|c|c|c|c|}
\hline & & & & & & LIEDIKNSB & \\
\hline imnoraphis_rob & L EKE? & LRSA & $D G Y E$ & LSVATN & HLGHF LICNL & MLEDLKHSG- & 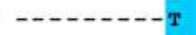 \\
\hline Synechocystis & L LDE & IพSA & DDYE & ISVATN & HL GHFLICNL & LIEDIKACP- & 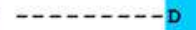 \\
\hline nab & $\operatorname{LIKE}$ & LRSP & $E G Y$ & ITMTIN & HIGBFLICKL & MIEDIQKSS- & ----- \\
\hline$r i$ & L IKE & LRSP & $E G Y$ & LTVTTN & HLGBPLICNL & MLEDLKKSS- & $-\cdots$ \\
\hline al & LAKE & QR SE & DGY & LSVATN & HLGBFLLCNL & MIEDIQKSS- & $-\cdots--$ \\
\hline & I SKE & LRNP & $E \subseteq Y$ & I SVATN & HLGBFLICNL & MIEDIKNSS- & ----- \\
\hline & L L K E & LRSV & & I SVATN & HLGBFLLCNL & MIEDLKKST- & -- \\
\hline & L LKE & MR & & VATN & $H I G B Y L I C R L$ & MIDDLMRSP- & ----- \\
\hline & LLK & LR & & I SMATN & HIGBEIISNI & $L I E D I K N S P-$ & \\
\hline & LI & LR & & LTVATN & HLGRFL & $M \perp E D I$ & \\
\hline & & & & & & & \\
\hline
\end{tabular}




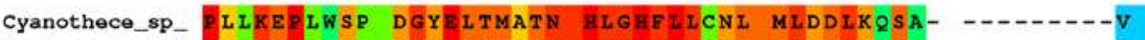
Nostoc_punctifo LIKEPLRSP EGYELTVTTN HLGHFLLCNL MLEDLKKSS Nicotiana_tabac TAKEPTFTA DGF LSVGTN HLGHFLISRL LLDDLKQSDZea mays TAKEPSYTA DGFEMSVGVN HLGHELIARE LLSDIOSSD- - Marchantia_palePTDKEPKFSA EGF LSVGTN HMGHFLLARL LMEDLQKAKPinus_mugo PTAKLPTYTA EGFELSVGTN HLGHELISRI LLEDIKTSDPhyscomitrella_PAKEPRYTA DGFELSVGTN HLGHELLANL LMEDIQHKER_-.-.-.N Chlamydomonas_r PAKEPRFTA DGFELSVGTN HLGHFLITNL LLDDLKNAP-
Daucus_carota

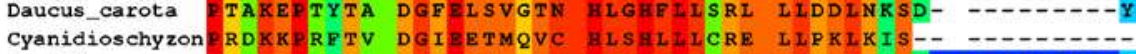
ProchlorococcusPRIKRPERSA QGFENSMAVN HFGHFIMINL LMENILSSER BIVLNGKSIV Consistency

$$
\text { R1 (loop 1) }
$$

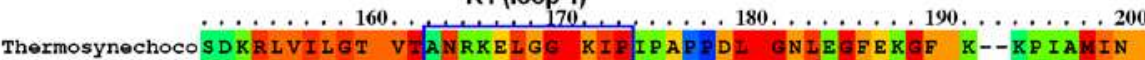
ThermosynechocoSDKRLVILGT VMANRKELGG KIPIPAPPDE GNLEGEEKGF K--KPIAMIN
Limnoraphis_robSQPRLIILGT VIANPKELGG KIPIPAPRD GNLQGLAGF K--APITMID Anabaena_variabADKRLVILGT VTHNPDELGG KIPPRP--D GNIBGFAQGF K--PPISMID Crinalium_epipsPDRRLVIIGT VMHNPDELGG KIPPRP--DE GNFEGFEAGF Q--EPISMID Calothrix_parasDAARLVILGT VIANPKELGG KIPIPAPRDI GDLQGEEAGF K--APISNIN KT DIPAPPD GDLKGEERE K- PPISHIN

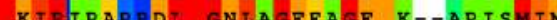
stanieria_cyanoSDKRLVILGT VAANPKELG KIPIPAPRD GNLEGEEAGF K--EPITMIN Microcystis_aerPDRRLVILGT VTHNPDELGG KIPPRP--DI GDIEGEAKGF K--EPITMAD OscillatorialeSEDKRLVILGT VIHNPDEIGG KIPPRP-DE GNLDGFAAGF K--APISMID Fischerella Cyanothece_sP_ KPAKLVIIGT VTHNPNELGG KIPPRP--DI GNIQGFAAGF K--APYSMID Nostoc_punctifOE-PRIVILGT VIHNPDEIGC KIPPRP--DE GDIQGFAEG K--EPISMID Nicotiana_tabacPQKRLIIVGS IIGNTNTLAG NVPPKA--NL GDLRGLSGGL NSLNCSPMID Zea_mays Marchantia_paleSLKRMIIVGS IIGNSNTVA Pinus_mugo NSKRVIIVGS ITGNTTIA Physcomitrella_NSRRVIIVGS IIGNTNTVA Chlamydomonas_rKQPRCIIVGS ITGNTNTIA Daucus_carota PSKRLIIVGS IIGNTNTLA Cyanidioschyzon-RGRIVELTT QTHSPNSIP Prochlorococcus FKPRITVLGT VAANYSELG Consistency

$4459889898 \quad 844955697$

$$
\text { YxxxK catalytic motif }
$$

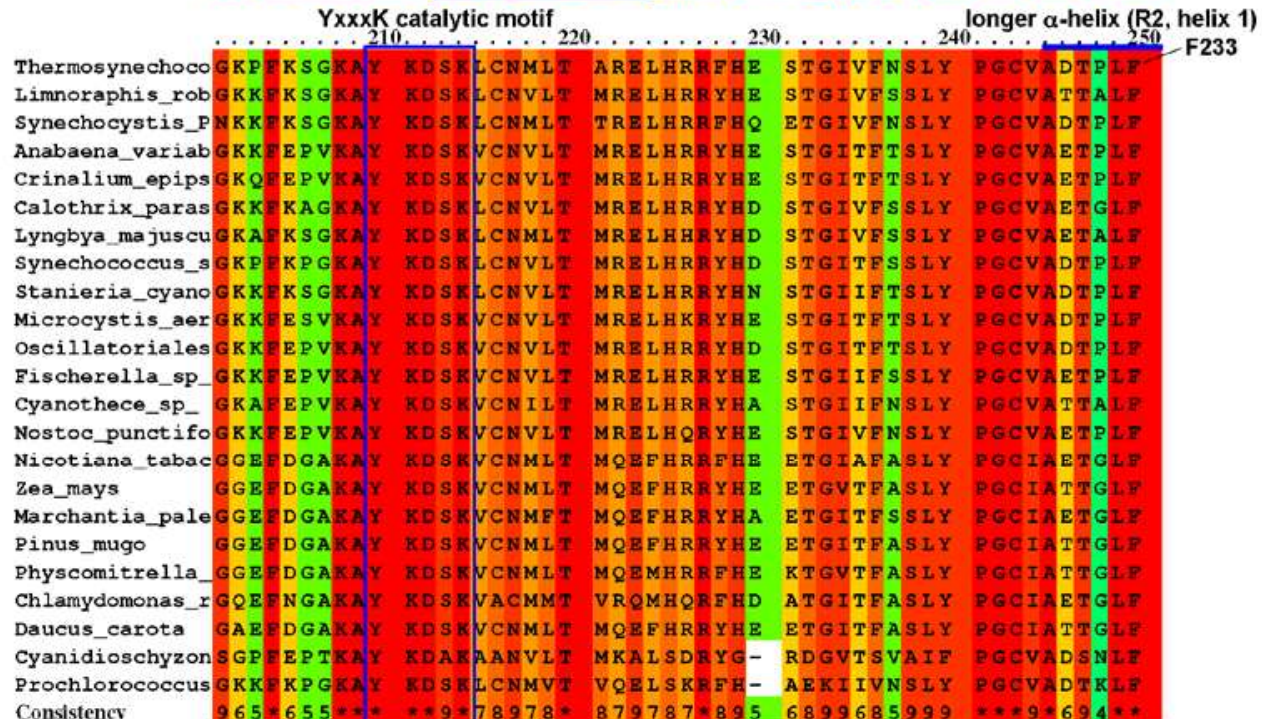
Consistency

\begin{tabular}{|c|c|c|c|c|c|}
\hline \multicolumn{6}{|c|}{260 . $270^{\circ}$} \\
\hline Thermosynechoco & H НF PLF $Q \mathrm{KL}$ & LFQKKIT & GGYVSQELAG & ERVAMVVADP & EFRQSGVHWS \\
\hline Limnoraphis_rob & $\mathrm{NHY} P \mathrm{~L}=\mathrm{QKI}$ & WFQKNII & GGFVSEELAG & ERVAMVVADP & EYNKSGVYWS \\
\hline Synechocystis_P & NHY SLPRTI & WFQKNVT & KGYVSQELAG & ERVAMVVADD & KFKDSGVHWS \\
\hline Anabaena_variab & NHYPLFQKI & LFQKYIT & GGYVSQBLAG & VADYIAAP & EYKQSGAYWS \\
\hline Crinalium_opips & NHYPLFOKI & $L F Q X Y I I$ & GGYVSQELSG & ERVAAVVADP & EYKQSGAYWS \\
\hline Calothrix_paras & NHY S L YQKI & LFQKNIT & GGYVSEBLAG & ERVAD V VADP & EYNKSGSYYS \\
\hline Lyngbya_majuscu & NHYSLFQKL & LFQNNIT & KGYVSQELAG & DRLATVVADP & NYNKS GVYWS \\
\hline Synechococcus_s & NHYPL $Q Q K L$ & $I F Q X N I T$ & GGYVSQELAG & $D R V A A V \vee A D P$ & ELKESGIYWS \\
\hline & NHY PLFRKI & & GGYVSQELAG & DRVAQVVADE & NYKESGAYFS \\
\hline
\end{tabular}

$$
\text { longer } \alpha \text {-helix (R2, helix 1) }
$$




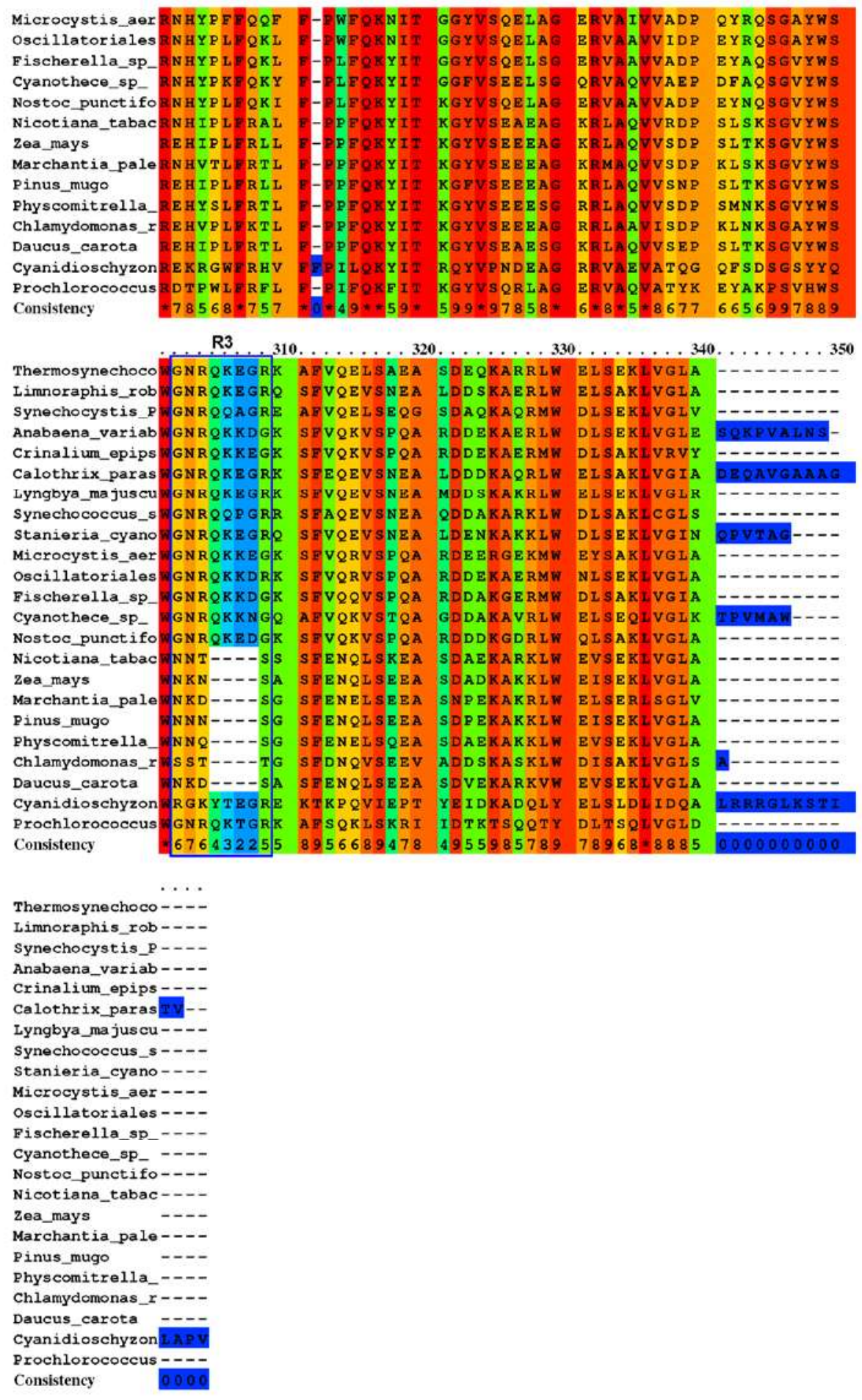

Figure

S1 Multiple sequence alignment of POR proteins from different organisms. Alignment of POR protein sequence from cyanobacteria and plants was carried out using the online PRALINE multiple server. The leader sequences from the plant PORs have been removed before alignment. 

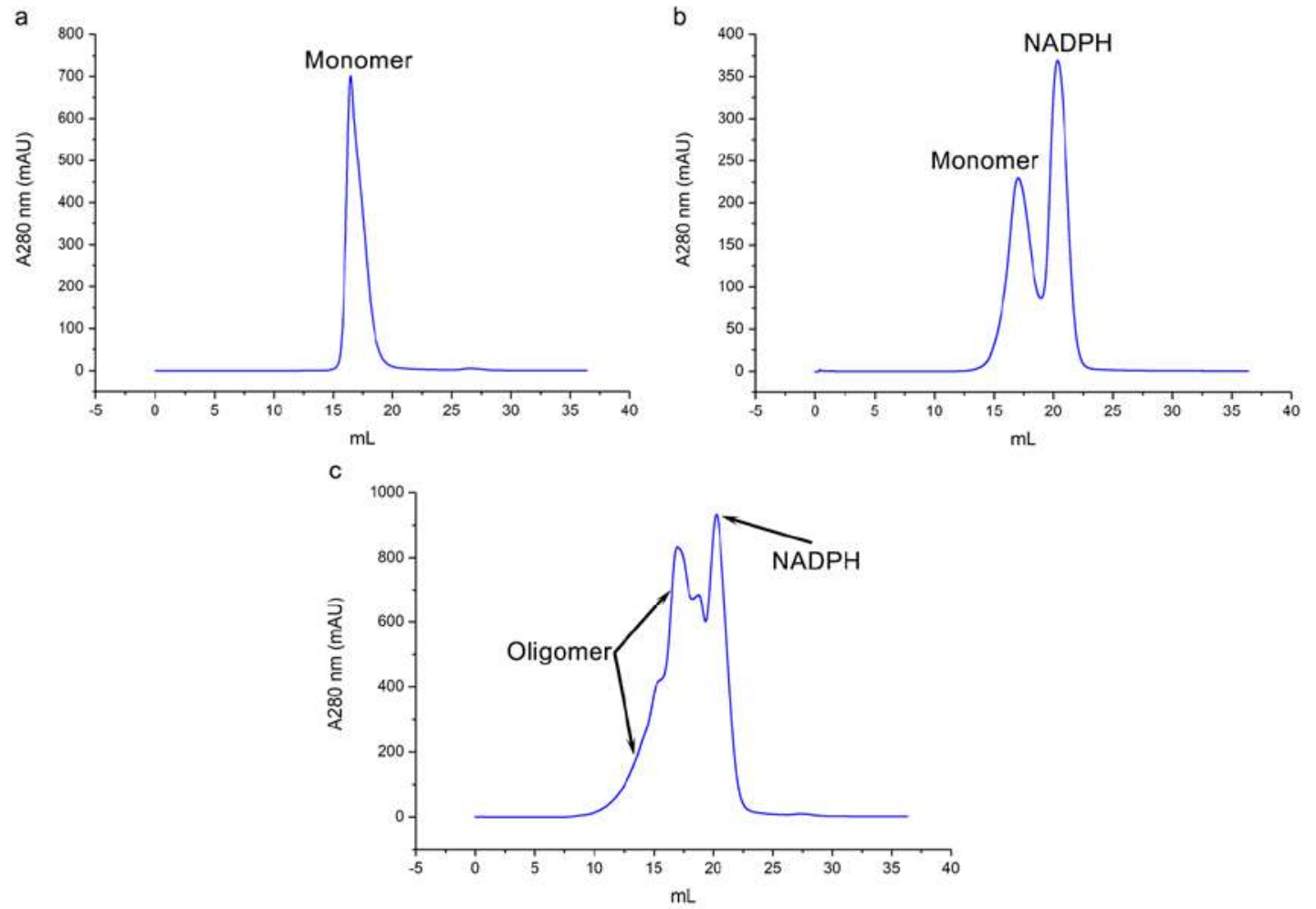

Figure S2. Elution profiles of $T$. elongatus POR after purification on gel filtration column. a) Purified $T$. elongatus POR alone. b) $T$. elongatus POR and NADPH c) $T$. elongatus POR ternary complex (T. elongatus POR with NADPH and Pchlide). 

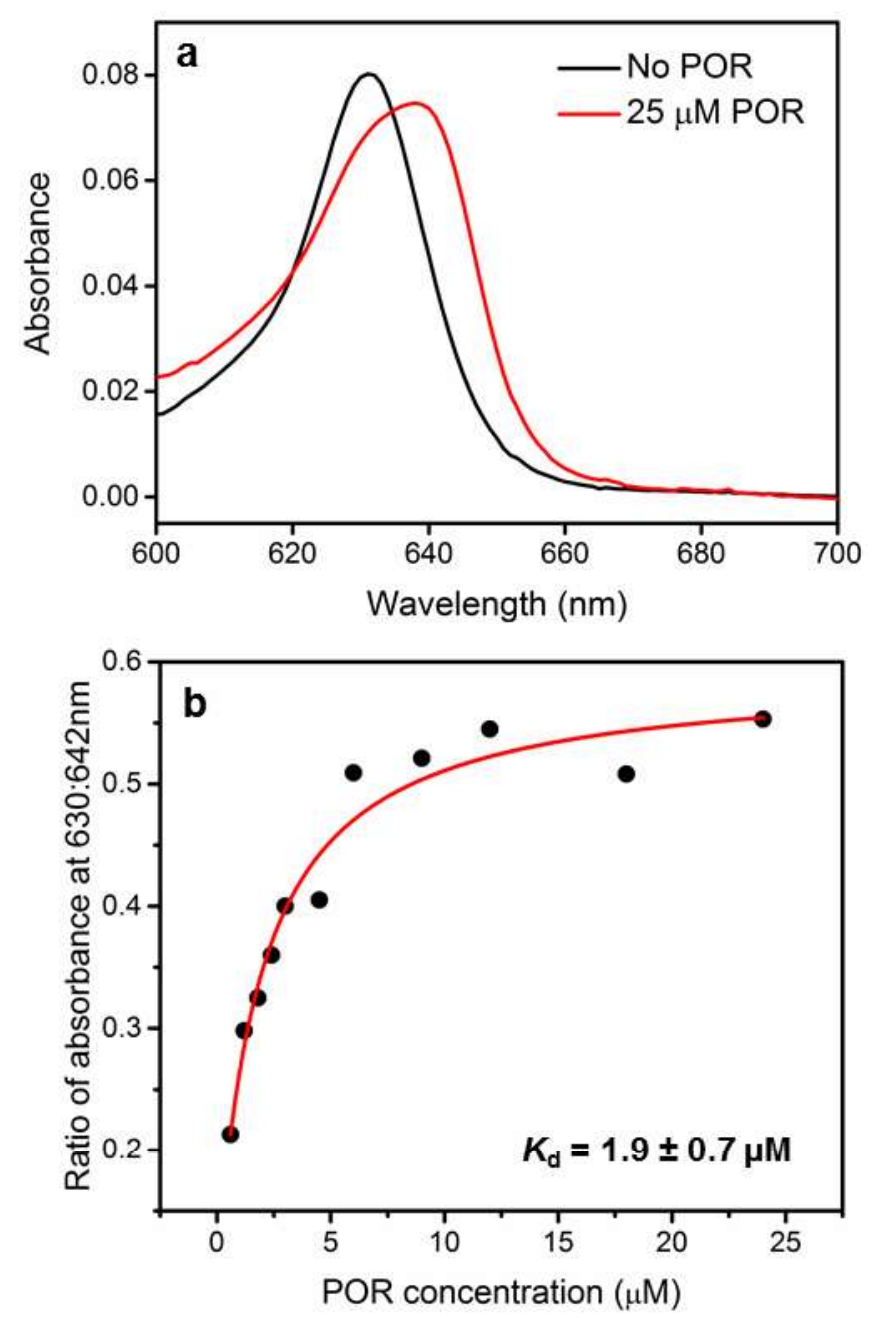

Figure S3. Analysis of spectral changes associated with Pchlide binding. (a) Absorbance spectra of Pchlide $(2.35 \mu \mathrm{M})$ in the presence and absence of either POR $(25 \mu \mathrm{M})+\mathrm{NADPH}$ $(250 \mu \mathrm{M})$. (b) The ratio of absorbance at $642 \mathrm{~nm}$ to $630 \mathrm{~nm}$ of Pchlide $(2.35 \mu \mathrm{M})$ measured at various POR concentrations in the presence of $250 \mu \mathrm{M}$ NADPH. All measurements were recorded at $25^{\circ} \mathrm{C}$. 

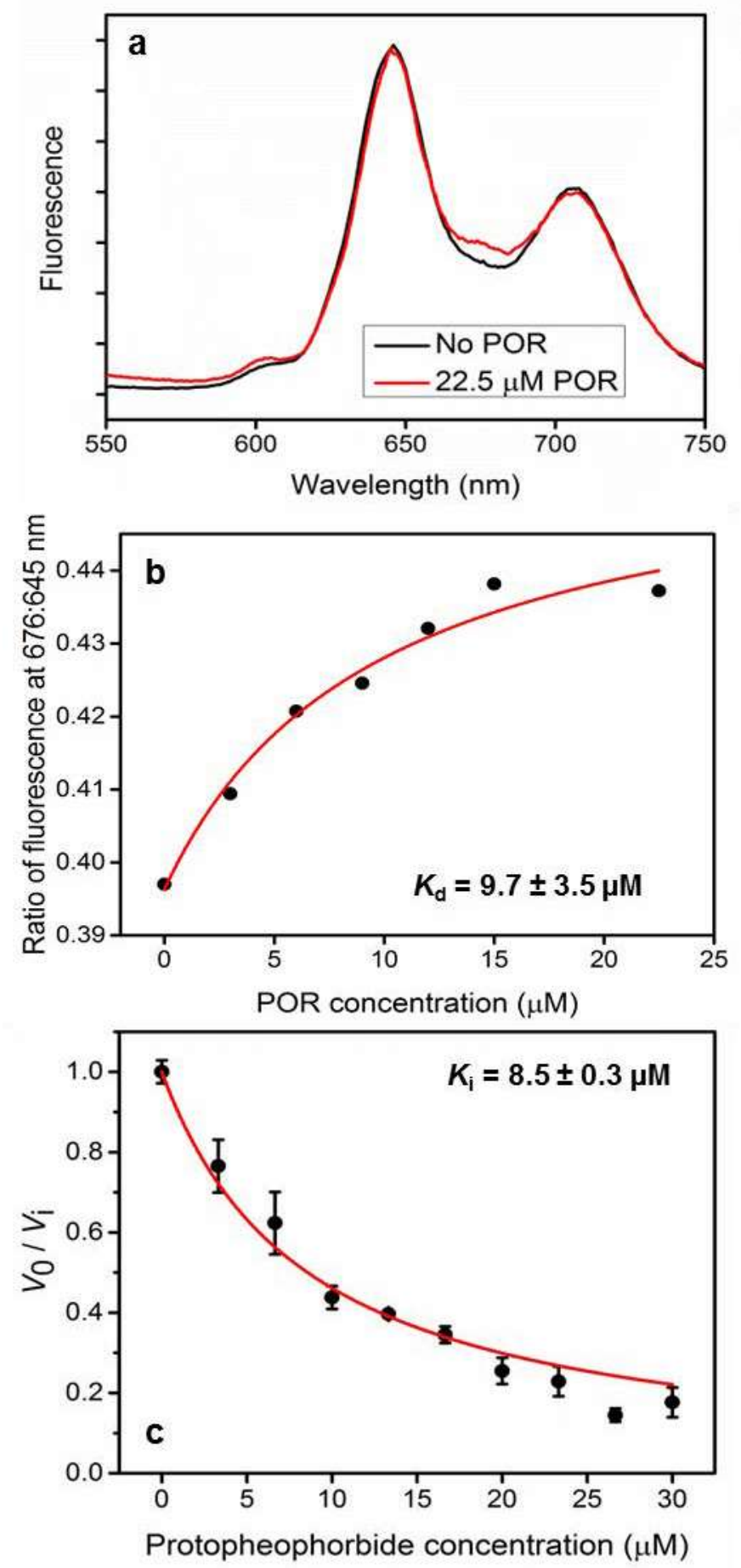

Figure S4. Analysis of spectral changes associated with protopheophorbide binding. (a)

Fluorescence emission spectra of protopheophorbide $(0.5 \mu \mathrm{M})$ in the presence and absence of either POR $(22.5 \mu \mathrm{M})+\mathrm{NADPH}(250 \mu \mathrm{M})$. (b) The ratio of fluorescence at $676 \mathrm{~nm}$ to 645 nm of protopheophorbide $(0.5 \mu \mathrm{M})$ measured at various POR concentrations in the presence of $250 \mu \mathrm{M}$ NADPH. (c) Inhibition of POR activity at increasing concentrations of protopheophorbide. 


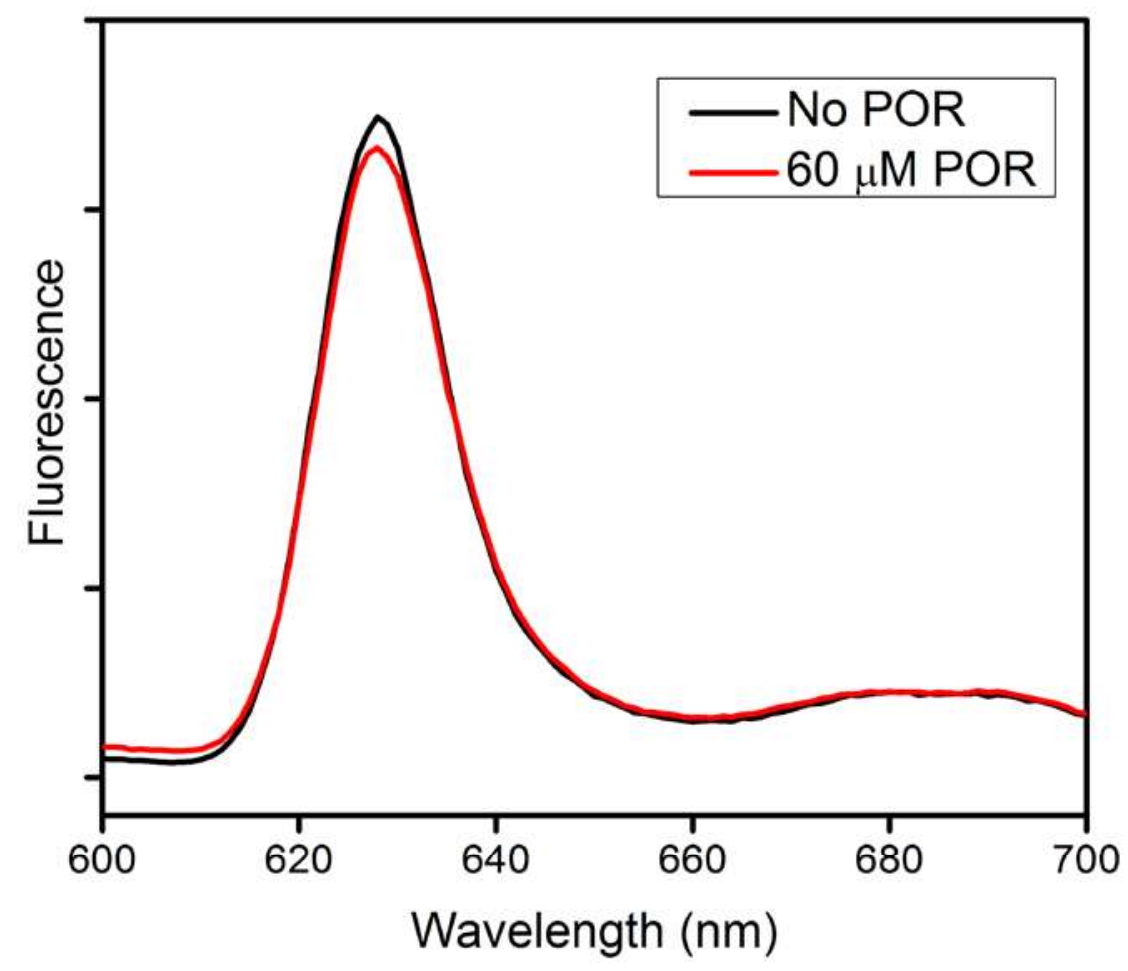

Figure S5. Analysis of spectral changes associated with $\mathrm{C} 17$ methylester Pchlide binding.

(a) Fluorescence emission spectra of $\mathrm{C} 17$ methylester Pchlide $(0.5 \mu \mathrm{M})$ in the presence and absence of either POR $(60 \mu \mathrm{M})+$ NADPH $(250 \mu \mathrm{M})$. No spectral changes could be observed $<250 \mu \mathrm{M}$ POR and no measurable inhibition of POR activity was detected at increasing $\mathrm{C} 17$ methylester Pchlide concentrations. 

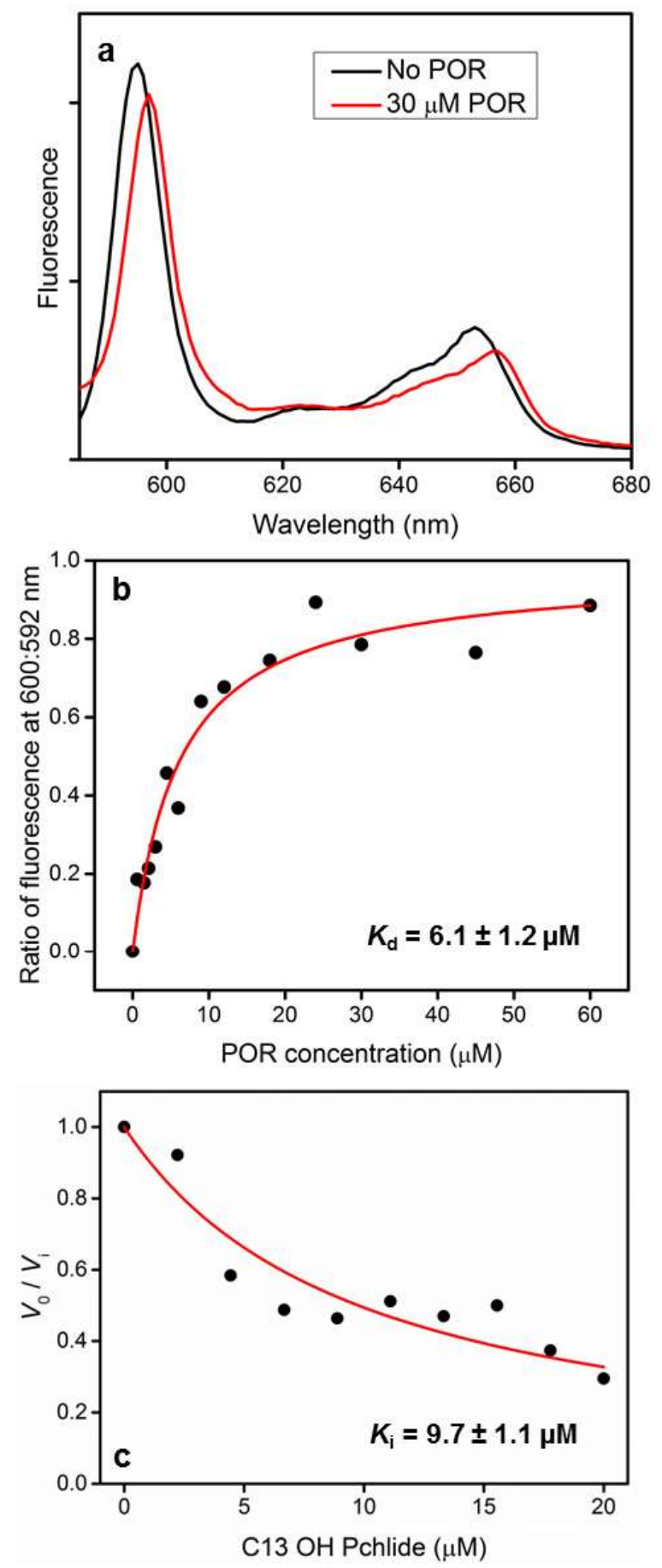

Figure S6. Analysis of spectral changes associated with $\mathrm{C13OH}$ Pchlide binding. (a) Fluorescence emission spectra of $\mathrm{C} 13 \mathrm{OH}$ Pchlide $(0.5 \mu \mathrm{M})$ in the presence and absence of either POR $(30 \mu \mathrm{M})+$ NADPH $(250 \mu \mathrm{M})$. (b) The ratio of fluorescence at $600 \mathrm{~nm}$ to $592 \mathrm{~nm}$ of $\mathrm{C} 13 \mathrm{OH}$ Pchlide $(0.5 \mu \mathrm{M})$ measured at various POR concentrations in the presence of 250 $\mu \mathrm{M}$ NADPH. (c) Inhibition of POR activity at increasing concentrations of $\mathrm{C} 13 \mathrm{OH}$ Pchlide. 

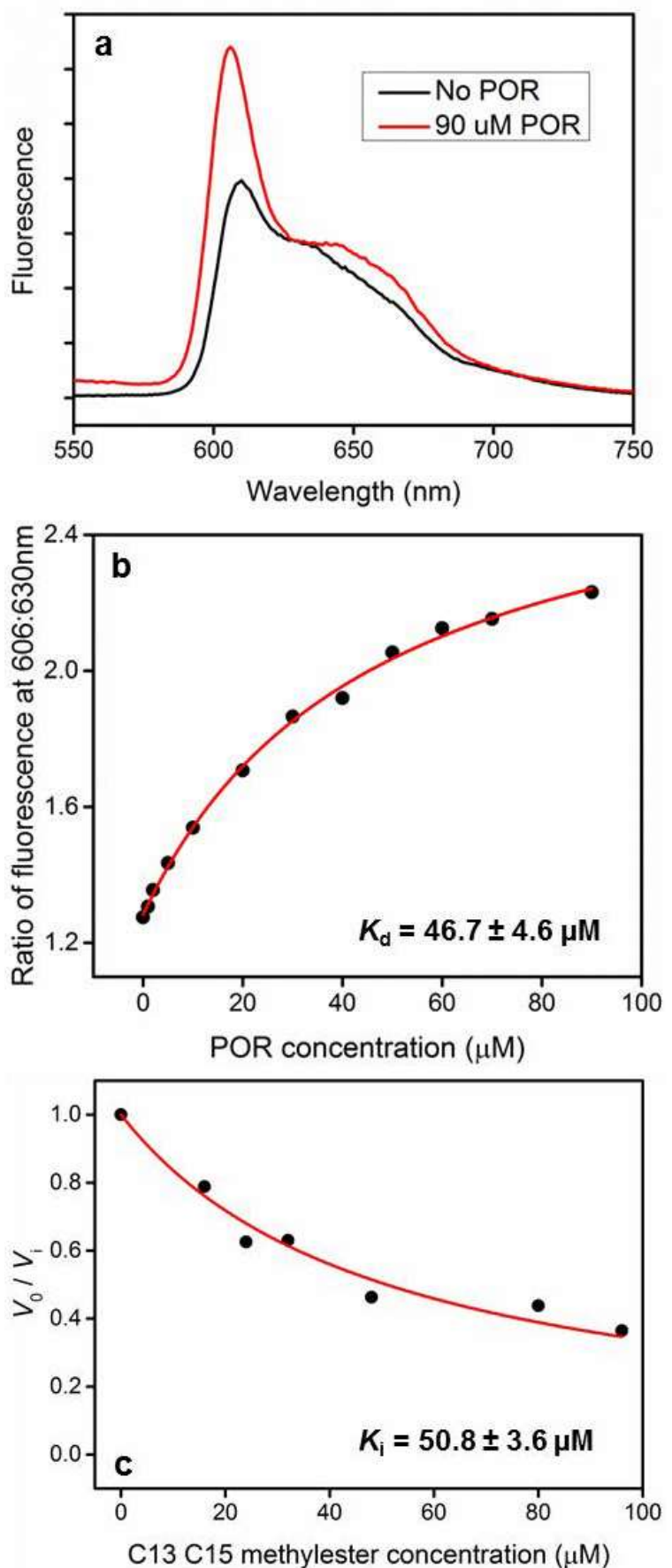

Figure S7. Analysis of spectral changes associated with C13C15 methylester Pchlide

binding. (a) Fluorescence emission spectra of $\mathrm{C} 13 \mathrm{C} 15$ methylester Pchlide $(0.5 \mu \mathrm{M})$ in the presence and absence of either POR $(30 \mu \mathrm{M})+\mathrm{NADPH}(250 \mu \mathrm{M})$. (b) The ratio of fluorescence at $600 \mathrm{~nm}$ to $592 \mathrm{~nm}$ of $\mathrm{C} 13 \mathrm{C} 15$ methylester Pchlide $(0.5 \mu \mathrm{M})$ measured at various POR concentrations in the presence of $250 \mu \mathrm{M}$ NADPH. (c) Inhibition of POR activity at increasing concentrations of $\mathrm{C} 13 \mathrm{C} 15$ methylester Pchlide. 

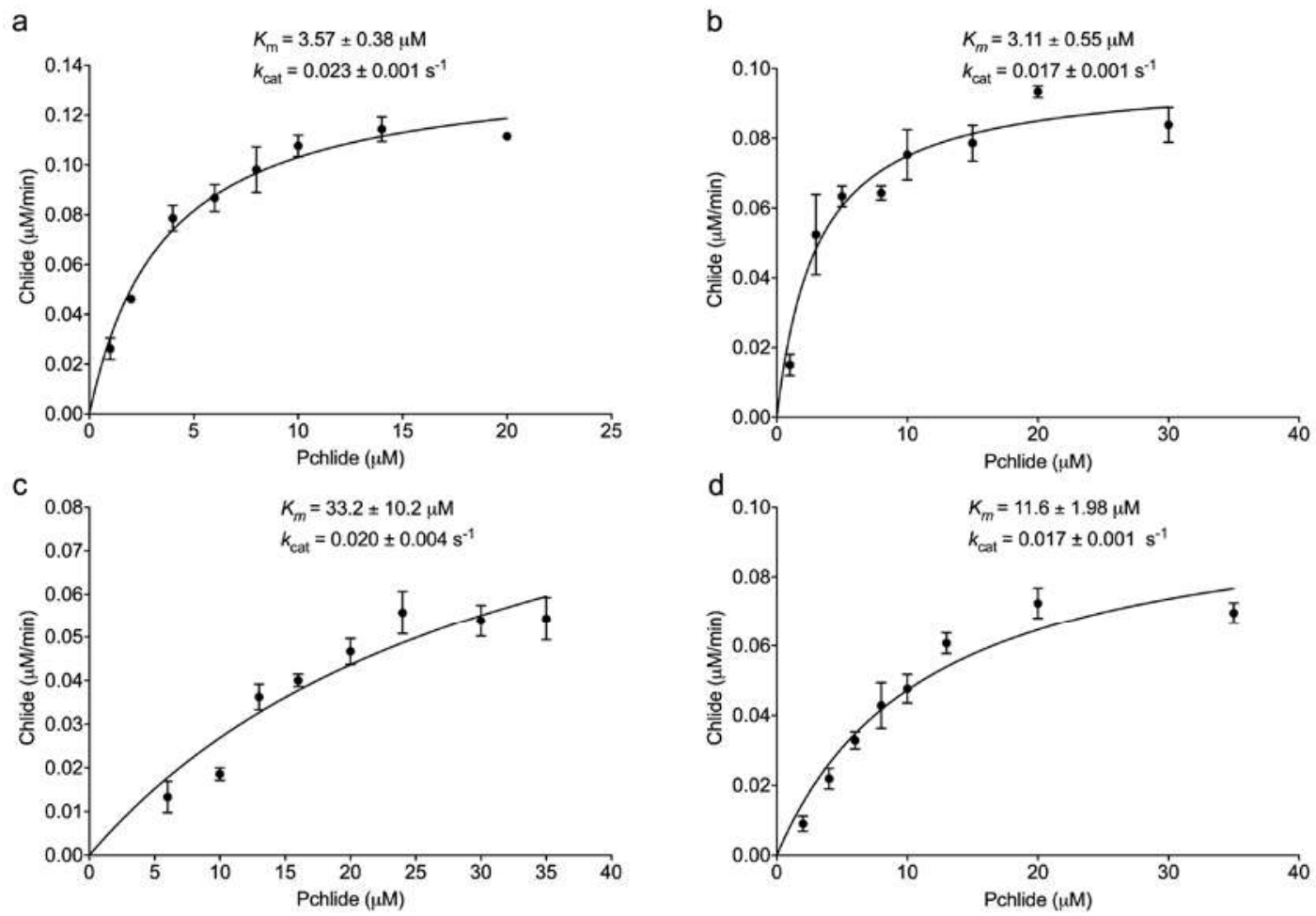

Figure S8. Steady-state activity of wild type and selected variants of $T$. elongatus POR.

The steady-state activity was measured over a range of Pchlide concentrations for (a) wild type, (b) K156A variant, (c) Y223A variant and (d) Y223F variant. All data were fitted to the Michaelis-Menten equation to obtain $K_{\mathrm{m}}$ and $k_{\text {cat }}$ values as described in the Methods. The values shown in the figure are mean \pm standard deviation, $n=3$. 

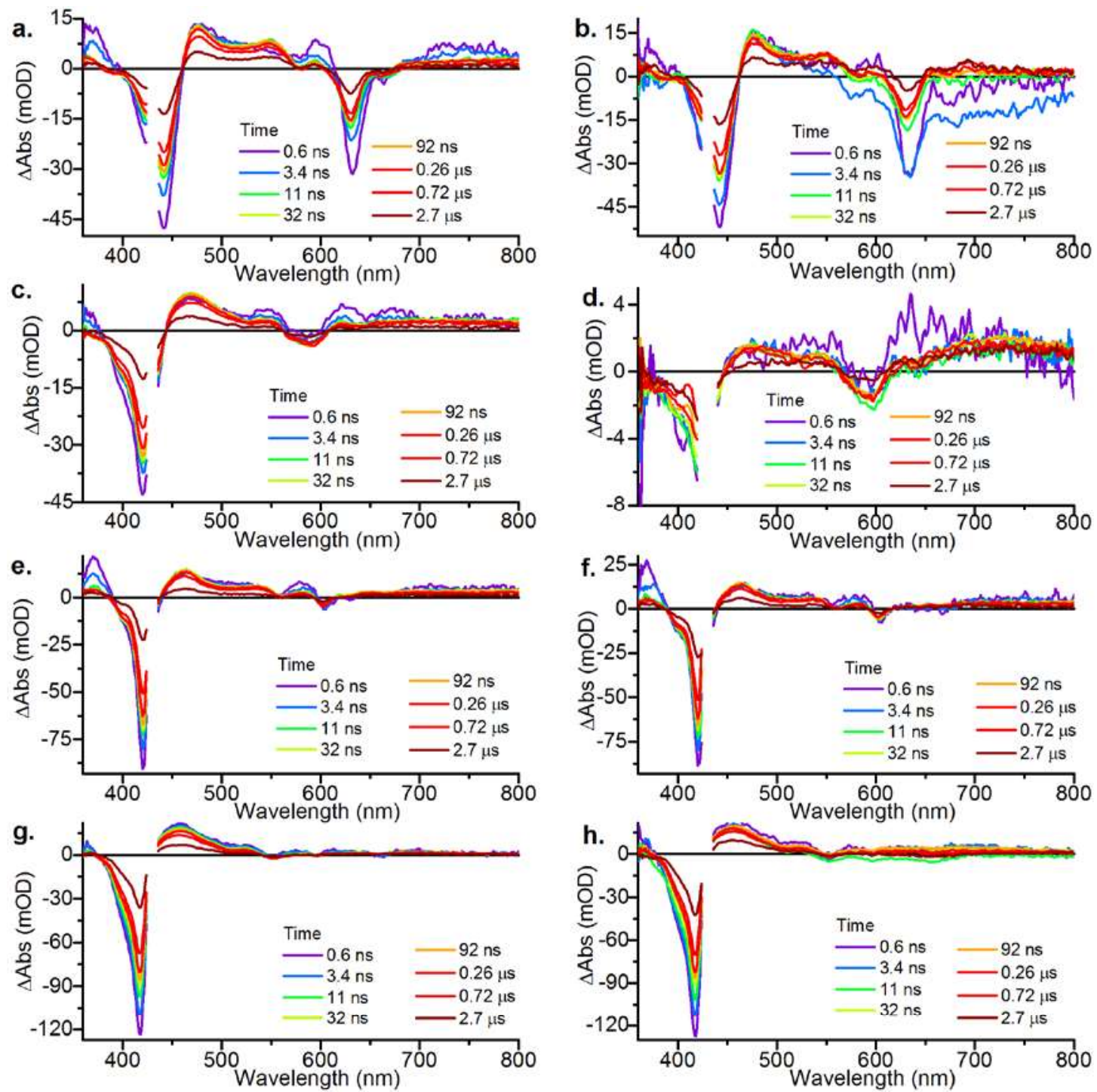

Figure S9. Visible transient absorption spectra at selected time delays after

photoexcitation at $430 \mathrm{~nm}$. Time-resolved visible spectroscopy data recorded between $0.6 \mathrm{~ns}$ and $2.7 \mu$ s for Pchlide (a), POR with NADPH and Pchlide (b), protopheophorbide (c), POR with NADPH and protopheophorbide (d), C13C15 methylester pchlide (e), POR with NADPH and C13C15 methylester pchlide (f), C13OH Pchlide (g), POR with NADPH and C13OH Pchlide (h). 
a
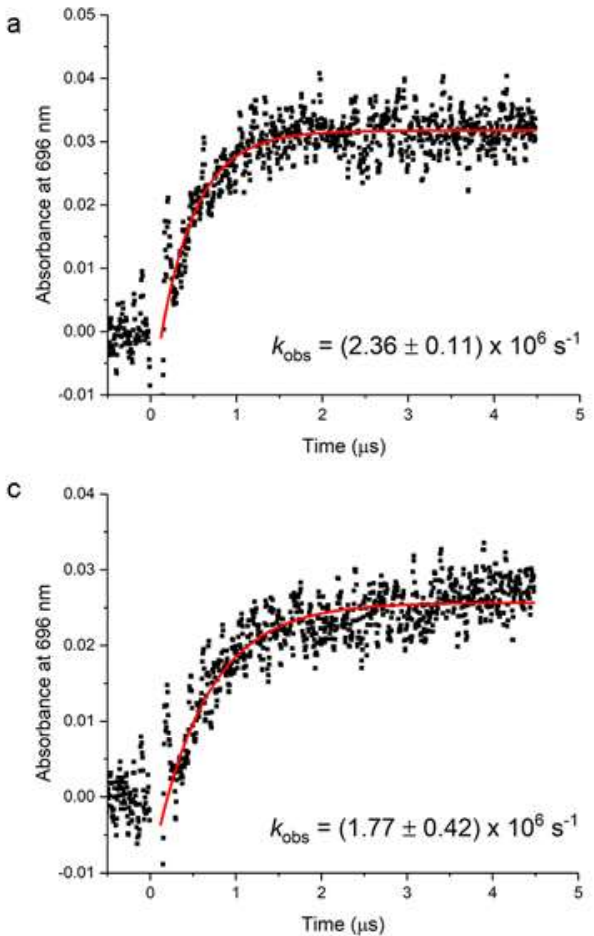

b

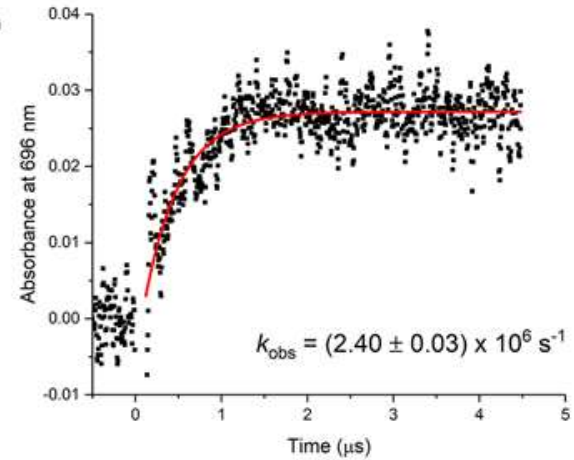

d

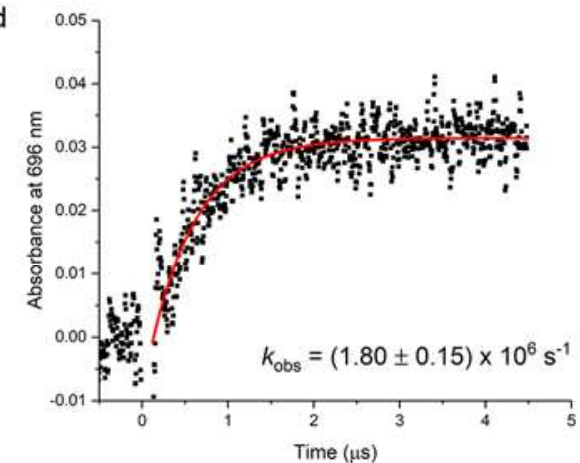

Figure S10. Kinetic transients of hydride transfer for wild type and selected variants of

T. elongatus POR. The rate of increase in absorbance at $696 \mathrm{~nm}$, corresponding to hydride transfer, measured for (a) wild type, (b) K156A variant, (c) Y223A variant and (d) Y223F variant. All data were fitted to a single exponential equation to obtain observed rate constants as described in the Methods. All the measurements were repeated three to five time, the values shown in the figure are mean \pm standard deviation, $n=5$. 

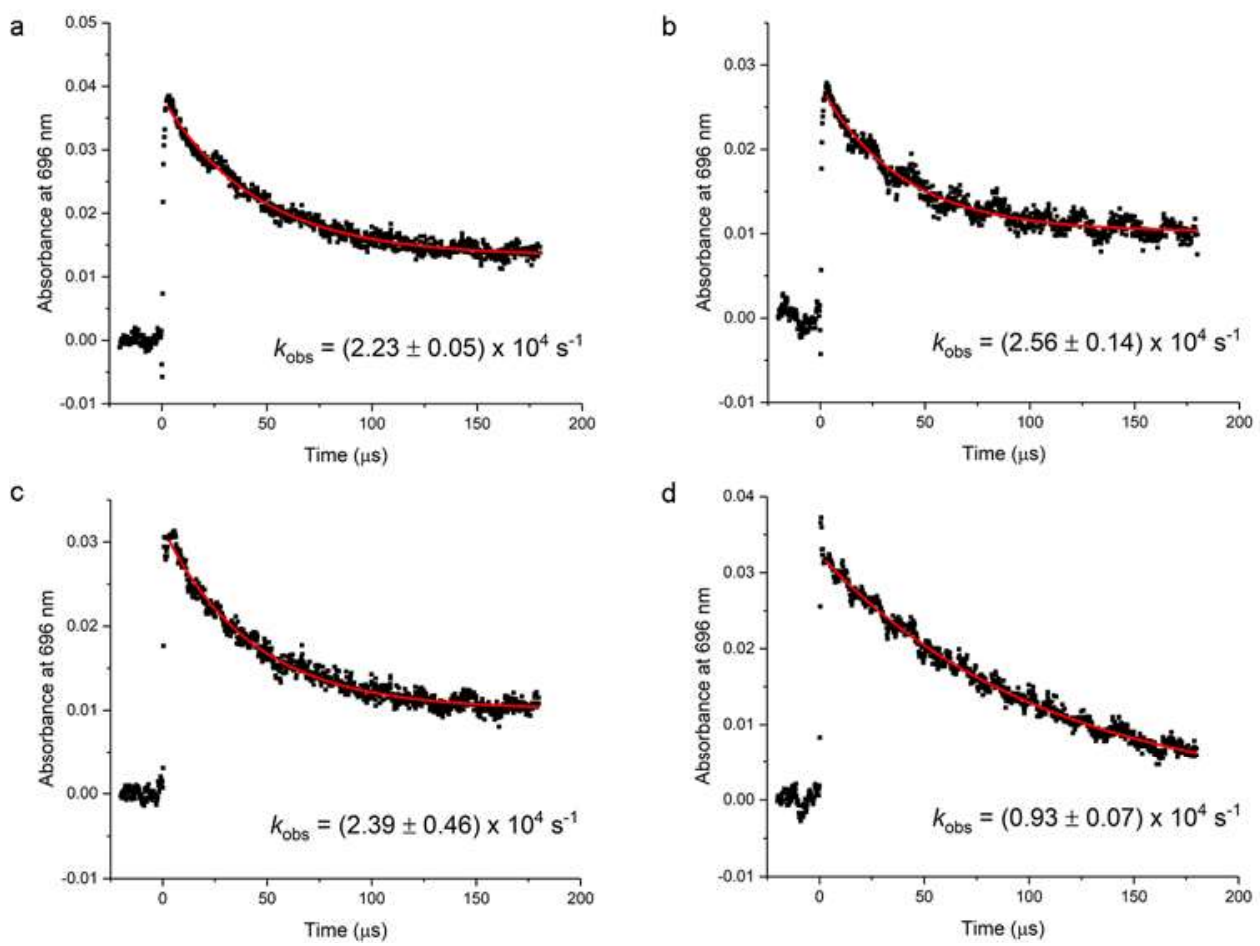

Figure S11. Kinetic transients of proton transfer for wild type and selected variants of $T$.

elongatus POR. The rate of decrease in absorbance at $696 \mathrm{~nm}$, corresponding to proton transfer, measured for (a) wild type, (b) K156A variant, (c) Y223A variant and (d) Y223F variant. All data were fitted to a single exponential equation to obtain observed rate constants as described in the Methods. All the measurements were repeated three to five time, the values shown in the figure are mean \pm standard deviation, $n=5$. 

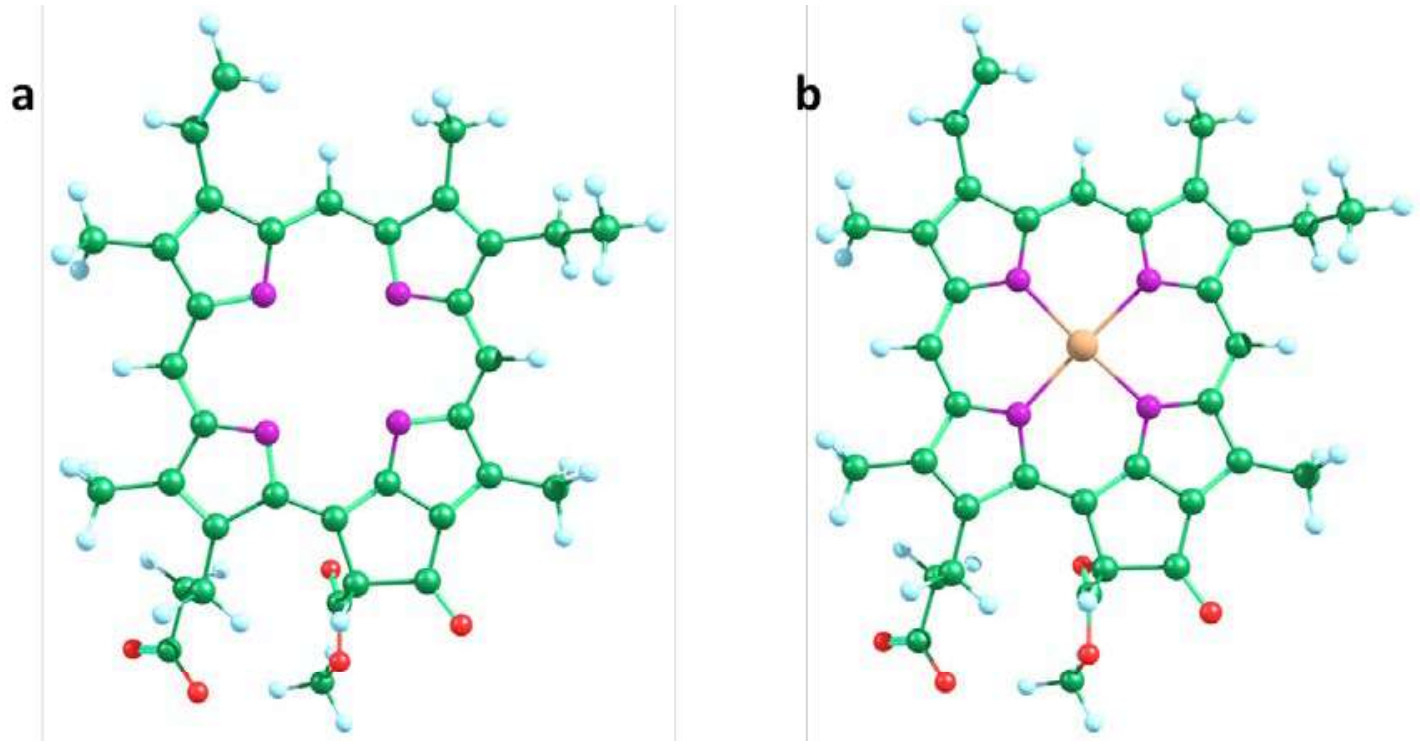

Figure

S12. DFT structures for parametrization of Pchlide. (a) Protopheophorbide and (b) Pchlide, optimized with the B3LYP functional and the LANL2DZ basis set for Mg and 6$31 \mathrm{G}^{*}$ for all other atoms.
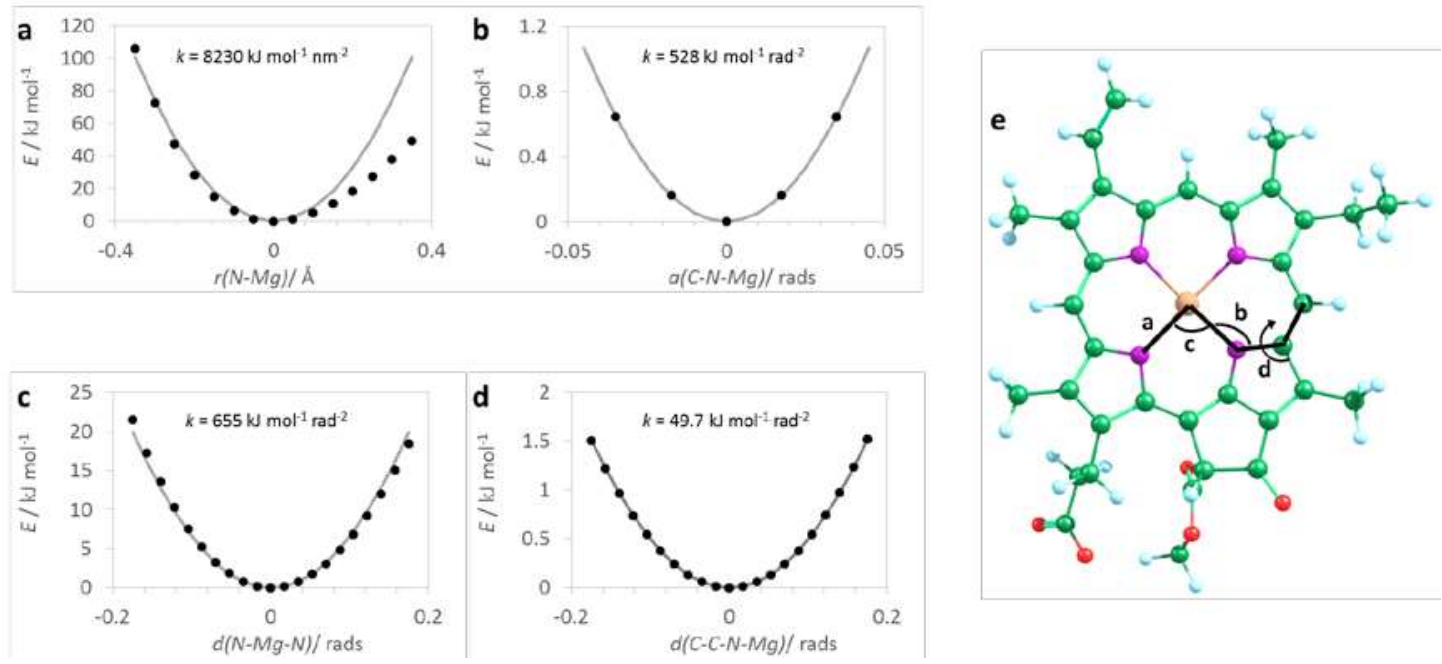

Figure S13. DFT scans for parameterization of Pchlide $\mathrm{Mg}^{2+}$ bonding parameters. (a) NMg distance, (b) C-N-Mg angle, (c) N-Mg-N angle and (d) C-C-N-Mg dihedral, as defined in (e). Force constants, $k$, are shown in each plot. Note that the MM parameters do not treat anharmonicity, only the quadratic portion of the plot in (a) was included in the fitting. 


\section{Supplementary Tables}

Table S1. Primers used for T. elongatus POR variants.

\begin{tabular}{ll}
\hline Primer & Sequence 5' to 3' \\
\hline K156A-F & TAAAGAATTAGGTGGCGCAATTCCGATTCCGGCA \\
K156A-R & TGCCGGAATCGGAATTGCGCCACCTAATTCTTTA \\
Y223A-F & GCATTGTGTTTAATAGCCTGGCTCCGGGTTGTGTTGCAGATA \\
Y223A-R & TATCTGCAACACAACCCGGAGCCAGGCTATTAAACACAATGC \\
Y223F-F & TTGTGTTTAATAGCCTGTTTCCGGGTTGTGTTGCAGATA \\
Y223F-R & TATCTGCAACACAACCCGGAAACAGGCTATTAAACACAA \\
\hline
\end{tabular}




\section{MD Parameters for NADPH and PChlide}

NADPH parameters from ANTECHAMBER and DFT modelling: [atoms ]

\begin{tabular}{|c|c|c|c|c|c|c|c|c|c|c|}
\hline $\mathrm{nr}$ & type & resnr & residue & atom & cgnr & charge & mass & & & \\
\hline 5050 & $\mathrm{P}$ & 1 & NDP & P1 & 1 & 1.310519 & 30.97 & ; & qtot & 1.3105 \\
\hline 5051 & os & 1 & NDP & 05 & 1 & -0.59251 & 16 & ; & qtot & 0.718 \\
\hline 5052 & $\mathrm{CT}$ & 1 & NDP & $\mathrm{C} 4$ & 1 & 0.252361 & 12.01 & ; & qtot & 0.9704 \\
\hline 5053 & CT & 1 & NDP & $\mathrm{C} 1$ & 1 & 0.819891 & 12.01 & ; & qtot & 1.7903 \\
\hline 5054 & OS & 1 & NDP & 013 & 1 & -0.787369 & 16 & ; & qtot & 1.0029 \\
\hline 5055 & CT & 1 & NDP & C11 & 1 & 0.491614 & 12.01 & ; & qtot & 1.4945 \\
\hline 5056 & $\mathrm{CT}$ & 1 & NDP & C7 & 1 & -0.072198 & 12.01 & ; & qtot & 1.4223 \\
\hline 5057 & $\mathrm{OH}$ & 1 & NDP & 010 & 1 & -0.75739 & 16 & ; & qtot & 0.6649 \\
\hline 5058 & $\mathrm{HO}$ & 1 & NDP & $\mathrm{H} 10$ & 1 & 0.486022 & 1.008 & ; & qtot & 1.1509 \\
\hline 5059 & $\mathrm{H} 1$ & 1 & NDP & H8 & 1 & 0.183749 & 1.008 & ; & qtot & 1.3347 \\
\hline 5060 & $\mathrm{CT}$ & 1 & NDP & C15 & 1 & -0.116034 & 12.01 & ; & qtot & 1.2187 \\
\hline 5061 & OS & 1 & NDP & 015 & 1 & -0.409514 & 16 & ; & qtot & 0.8091 \\
\hline 5062 & $\mathrm{P}$ & 1 & NDP & P2 & 1 & 1.224101 & 30.97 & ; & qtot & 2.0332 \\
\hline 5063 & $\mathrm{O} 2$ & 1 & NDP & 01 & 1 & -0.797153 & 16 & ; & qtot & 1.2361 \\
\hline 5064 & $\mathrm{O} 2$ & 1 & NDP & 04 & 1 & -0.797153 & 16 & ; & qtot & 0.4389 \\
\hline 5065 & os & 1 & NDP & 09 & 1 & -0.600853 & 16 & ; & qtot & -0.1619 \\
\hline 5066 & $P$ & 1 & NDP & P3 & 1 & 1.383435 & 30.97 & ; & qtot & 1.2215 \\
\hline 5067 & $\mathrm{O} 2$ & 1 & NDP & $\mathrm{O} 2$ & 1 & -0.806494 & 16 & ; & qtot & 0.415 \\
\hline 5068 & $\mathrm{O} 2$ & 1 & NDP & 07 & 1 & -0.806494 & 16 & ; & qtot & -0.3915 \\
\hline 5069 & OS & 1 & NDP & 016 & 1 & -0.562829 & 16 & ; & qtot & -0.9543 \\
\hline 5070 & CT & 1 & NDP & C16 & 1 & 0.144251 & 12.01 & ; & qtot & -0.81 \\
\hline 5071 & $\mathrm{CT}$ & 1 & NDP & $\mathrm{C} 12$ & 1 & 0.041224 & 12.01 & ; & qtot & -0.7688 \\
\hline 5072 & CT & 1 & NDP & $\mathrm{C} 8$ & 1 & 0.477906 & 12.01 & ; & qtot & -0.2909 \\
\hline 5073 & $\mathrm{CT}$ & 1 & NDP & $\mathrm{C} 5$ & 1 & 0.106426 & 12.01 & ; & qtot & -0.1845 \\
\hline 5074 & CT & 1 & NDP & $\mathrm{C} 2$ & 1 & 0.071548 & 12.01 & ; & qtot & -0.1129 \\
\hline 5075 & NT & 1 & NDP & N2 & 1 & 0.152528 & 14.01 & ; & qtot & 0.0396 \\
\hline 5076 & $\mathrm{CM}$ & 1 & NDP & $\mathrm{C} 6$ & 1 & -0.106105 & 12.01 & ; & qtot & -0.0665 \\
\hline 5077 & CD & 1 & NDP & $\mathrm{C} 9$ & 1 & -0.252248 & 12.01 & ; & qtot & -0.3188 \\
\hline 5078 & CT & 1 & NDP & $\mathrm{C} 13$ & 1 & 0.190136 & 12.01 & ; & qtot & -0.1286 \\
\hline 5079 & $\mathrm{CM}$ & 1 & NDP & C17 & 1 & -0.242361 & 12.01 & ; & qtot & -0.371 \\
\hline 5080 & $\mathrm{CM}$ & 1 & NDP & C19 & 1 & -0.274995 & 12.01 & ; & qtot & -0.646 \\
\hline 5081 & HA & 1 & NDP & $\mathrm{H} 20$ & 1 & 0.157059 & 1.008 & ; & qtot & -0.4889 \\
\hline 5082 & HA & 1 & NDP & H19 & 1 & 0.090239 & 1.008 & ; & qtot & -0.3987 \\
\hline 5083 & $\mathrm{HC}$ & 1 & NDP & $\mathrm{H} 14$ & 1 & -0.021133 & 1.008 & ; & qtot & -0.4198 \\
\hline 5084 & $\mathrm{HC}$ & 1 & NDP & $\mathrm{H} 26$ & 1 & -0.021133 & 1.008 & ; & qtot & -0.441 \\
\hline 5085 & $\mathrm{C}$ & 1 & NDP & $\mathrm{C} 20$ & 1 & 0.866452 & 12.01 & ; & qtot & 0.4255 \\
\hline 5086 & $\mathrm{~N}$ & 1 & NDP & N6 & 1 & $-1,046682$ & 14.01 & ; & qtot & -0.6212 \\
\hline 5087 & $\mathrm{H}$ & 1 & NDP & $\mathrm{H} 23$ & 1 & 0.436516 & 1.008 & ; & qtot & -0.1847 \\
\hline 5088 & $\mathrm{H}$ & 1 & NDP & $\mathrm{H} 24$ & 1 & 0.436516 & 1.008 & ; & qtot & 0.2518 \\
\hline 5089 & 0 & 1 & NDP & 017 & 1 & -0.740088 & 16 & ; & qtot & -0.4882 \\
\hline 5090 & HA & 1 & NDP & $\mathrm{H} 6$ & 1 & 0.191704 & 1.008 & ; & qtot & -0.2965 \\
\hline 5091 & OS & 1 & NDP & 014 & 1 & -0.396099 & 16 & ; & qtot & -0.6926 \\
\hline 5092 & $\mathrm{H} 2$ & 1 & NDP & $\mathrm{H} 2$ & 1 & 0.12238 & 1.008 & ; & qtot & -0.5703 \\
\hline 5093 & $\mathrm{OH}$ & 1 & NDP & 06 & 1 & -0.680592 & 16 & ; & qtot & -1.2508 \\
\hline 5094 & $\mathrm{HO}$ & 1 & NDP & H7 & 1 & 0.410823 & 1.008 & ; & qtot & -0.84 \\
\hline 5095 & $\mathrm{H} 1$ & 1 & NDP & H5 & 1 & 0.099805 & 1.008 & ; & qtot & -0.7402 \\
\hline 5096 & $\mathrm{OH}$ & 1 & NDP & 011 & 1 & -0.713702 & 16 & ; & qtot & -1.4539 \\
\hline 5097 & $\mathrm{HO}$ & 1 & NDP & H11 & 1 & 0.406809 & 1.008 & ; & qtot & -1.0471 \\
\hline 5098 & $\mathrm{H} 1$ & 1 & NDP & H9 & 1 & -0.001476 & 1.008 & ; & qtot & -1.0486 \\
\hline 5099 & $\mathrm{H} 1$ & 1 & NDP & $\mathrm{H} 13$ & 1 & 0.029745 & 1.008 & ; & qtot & -1.0188 \\
\hline 5100 & $\mathrm{H} 1$ & 1 & NDP & $\mathrm{H} 17$ & 1 & 0.045688 & 1.008 & ; & qtot & -0.9732 \\
\hline 5101 & $\mathrm{H} 1$ & 1 & NDP & H18 & 1 & 0.045688 & 1.008 & ; & qtot & -0.9275 \\
\hline 5102 & $\mathrm{H} 1$ & 1 & NDP & H15 & 1 & 0.084308 & 1.008 & ; & qtot & -0.8432 \\
\hline 5103 & $\mathrm{H} 1$ & 1 & NDP & H16 & 1 & 0.084308 & 1.008 & ; & qtot & -0.7589 \\
\hline 5104 & $\mathrm{H} 1$ & 1 & NDP & H12 & 1 & 0.007635 & 1.008 & ; & qtot & -0.7512 \\
\hline 5105 & $\mathrm{~N}^{*}$ & 1 & NDP & N7 & 1 & -0.429578 & 14.01 & ; & qtot & -1.1808 \\
\hline 5106 & $\mathrm{CB}$ & 1 & NDP & C10 & 1 & 0.522821 & 12.01 & ; & qtot & -0.658 \\
\hline 5107 & NC & 1 & NDP & N3 & 1 & -0.739196 & 14.01 & ; & qtot & -1.3972 \\
\hline 5108 & CQ & 1 & NDP & $\mathrm{C} 3$ & 1 & 0.580157 & 12.01 & ; & qtot & -0.817 \\
\hline 5109 & $\mathrm{NC}$ & 1 & NDP & $\mathrm{N} 1$ & 1 & -0.823995 & 14.01 & ; & qtot & -1.641 \\
\hline 5110 & $\mathrm{CA}$ & 1 & NDP & C18 & 1 & 0.636546 & 12.01 & ; & qtot & -1.0045 \\
\hline 5111 & $\mathrm{CB}$ & 1 & NDP & $\mathrm{C} 14$ & 1 & 0.03376 & 12.01 & ; & qtot & -0.9707 \\
\hline 5112 & NB & 1 & NDP & N5 & 1 & -0.661122 & 14.01 & ; & qtot & -1.6318 \\
\hline 5113 & CK & 1 & NDP & $\mathrm{C} 21$ & 1 & 0.347285 & 12.01 & ; & qtot & -1.2845 \\
\hline 5114 & H5 & 1 & NDP & $\mathrm{H} 25$ & 1 & 0.138852 & 1.008 & ; & qtot & -1.1457 \\
\hline 5115 & $\mathrm{~N} 2$ & 1 & NDP & N4 & 1 & -0.791635 & 14.01 & ; & qtot & -1.9373 \\
\hline 5116 & $\mathrm{H}$ & 1 & NDP & $\mathrm{H} 21$ & 1 & 0.333133 & 1.008 & ; & qtot & -1.6042 \\
\hline 5117 & $\mathrm{H}$ & 1 & NDP & $\mathrm{H} 22$ & 1 & 0.333133 & 1.008 & ; & qtot & -1.2711 \\
\hline 5118 & H5 & 1 & NDP & $\mathrm{H} 3$ & 1 & 0.028062 & 1.008 & ; & qtot & -1.243 \\
\hline 5119 & $\mathrm{H} 2$ & 1 & NDP & $\mathrm{H} 1$ & 1 & -0.043089 & 1.008 & ; & qtot & -1.2861 \\
\hline 5120 & $\mathrm{H} 1$ & 1 & NDP & $\mathrm{H} 4$ & 1 & 0.046584 & 1.008 & ; & qtot & -1.2395 \\
\hline 5121 & $\mathrm{O} 2$ & 1 & NDP & 08 & 1 & -0.920166 & 16 & ; & q tot & -2.1597 \\
\hline 5122 & $\mathrm{O} 2$ & 1 & NDP & 012 & 1 & -0.920166 & 16 & ; & qtot & -3.0798 \\
\hline 5123 & $\mathrm{O} 2$ & 1 & NDP & 03 & 1 & -0.920166 & 16 & ; & qtot & -4 \\
\hline nds ] & & & & & & & & & & \\
\hline ai & aj & funct & $\mathrm{c} 0$ & c1 & c2 & c3 & & & & \\
\hline 5112 & 5113 & 1 & 0.1369 & & & & & & \\
\hline 5111 & 5112 & 1 & 0.1352 & \multicolumn{2}{|c|}{391371.36} & & & & & \\
\hline 5110 & 5111 & 1 & 0.1398 & \multicolumn{2}{|c|}{385848.48} & & & & & \\
\hline 5110 & 5115 & 1 & 0.1386 & 3496 & & & & & & \\
\hline
\end{tabular}




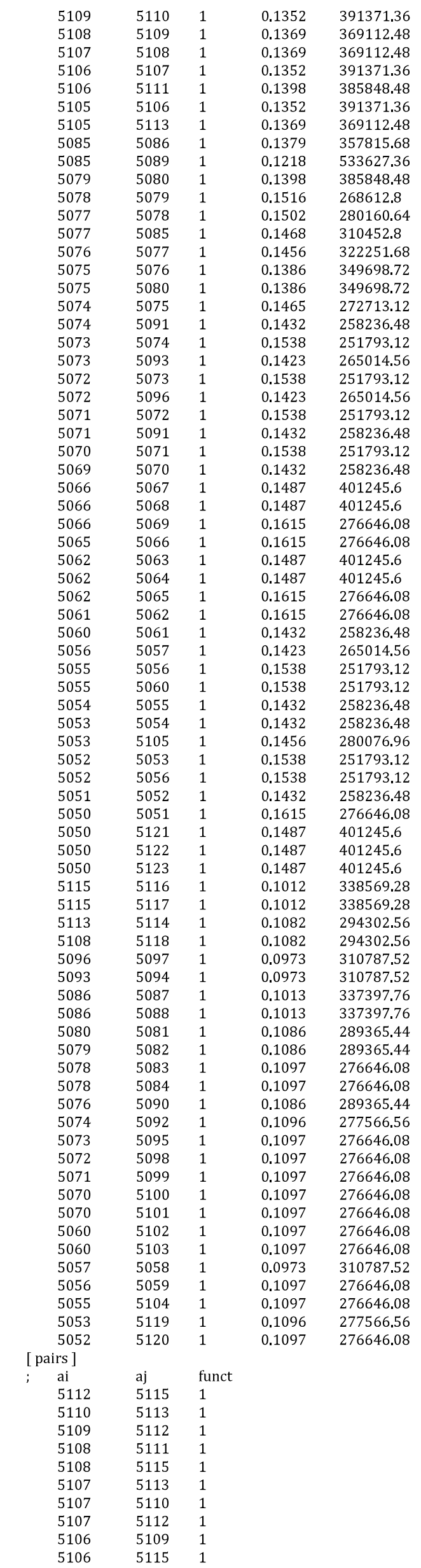




\begin{tabular}{|c|c|}
\hline 5105 & 5108 \\
\hline 5105 & 5110 \\
\hline 5093 & 5096 \\
\hline 5091 & 5093 \\
\hline 5091 & 5096 \\
\hline 5080 & 5091 \\
\hline 5079 & 5085 \\
\hline 5078 & 5086 \\
\hline 5078 & 5089 \\
\hline 5077 & 5080 \\
\hline 5076 & 5091 \\
\hline 5076 & 5079 \\
\hline 5076 & 5086 \\
\hline 5076 & 5089 \\
\hline 5075 & 5093 \\
\hline 5075 & 5078 \\
\hline 5075 & 5085 \\
\hline 5074 & 5096 \\
\hline 5074 & 5077 \\
\hline 5074 & 5079 \\
\hline 5073 & 5076 \\
\hline 5073 & 5080 \\
\hline 5072 & 5075 \\
\hline 5071 & 5093 \\
\hline 5071 & 5075 \\
\hline 5070 & 5073 \\
\hline 5070 & 5096 \\
\hline 5070 & 5074 \\
\hline 5069 & 5072 \\
\hline 5069 & 5091 \\
\hline 5068 & 5070 \\
\hline 5067 & 5070 \\
\hline 5066 & 5071 \\
\hline 5065 & 5070 \\
\hline 5064 & 5066 \\
\hline 5063 & 5066 \\
\hline 5062 & 5067 \\
\hline 5062 & 5068 \\
\hline 5062 & 5069 \\
\hline 5061 & 5066 \\
\hline 5060 & 5063 \\
\hline 5060 & 5064 \\
\hline 5060 & 5065 \\
\hline 5057 & 5060 \\
\hline 5056 & 5061 \\
\hline 5056 & 5105 \\
\hline 5055 & 5105 \\
\hline 5055 & 5062 \\
\hline 5054 & 5106 \\
\hline 5054 & 5113 \\
\hline 5054 & 5057 \\
\hline 5054 & 5061 \\
\hline 5053 & 5057 \\
\hline 5053 & 5060 \\
\hline 5053 & 5107 \\
\hline 5053 & 5111 \\
\hline 5053 & 5112 \\
\hline 5052 & 5121 \\
\hline 5052 & 5122 \\
\hline 5052 & 5123 \\
\hline 5052 & 5106 \\
\hline 5052 & 5113 \\
\hline 5052 & 5060 \\
\hline 5051 & 5054 \\
\hline 5051 & 5105 \\
\hline 5051 & 5055 \\
\hline 5051 & 5057 \\
\hline 5050 & 5053 \\
\hline 5050 & 5056 \\
\hline 5119 & 5120 \\
\hline 5113 & 5119 \\
\hline 5111 & 5116 \\
\hline 5111 & 5117 \\
\hline 5111 & 5114 \\
\hline 5110 & 5118 \\
\hline 5109 & 5116 \\
\hline 5109 & 5117 \\
\hline 5106 & 5119 \\
\hline 5106 & 5114 \\
\hline 5106 & 5118 \\
\hline 5105 & 5120 \\
\hline 5103 & 5104 \\
\hline 5102 & 5104 \\
\hline 5099 & 5100 \\
\hline 5099 & 5101 \\
\hline & \\
\hline
\end{tabular}




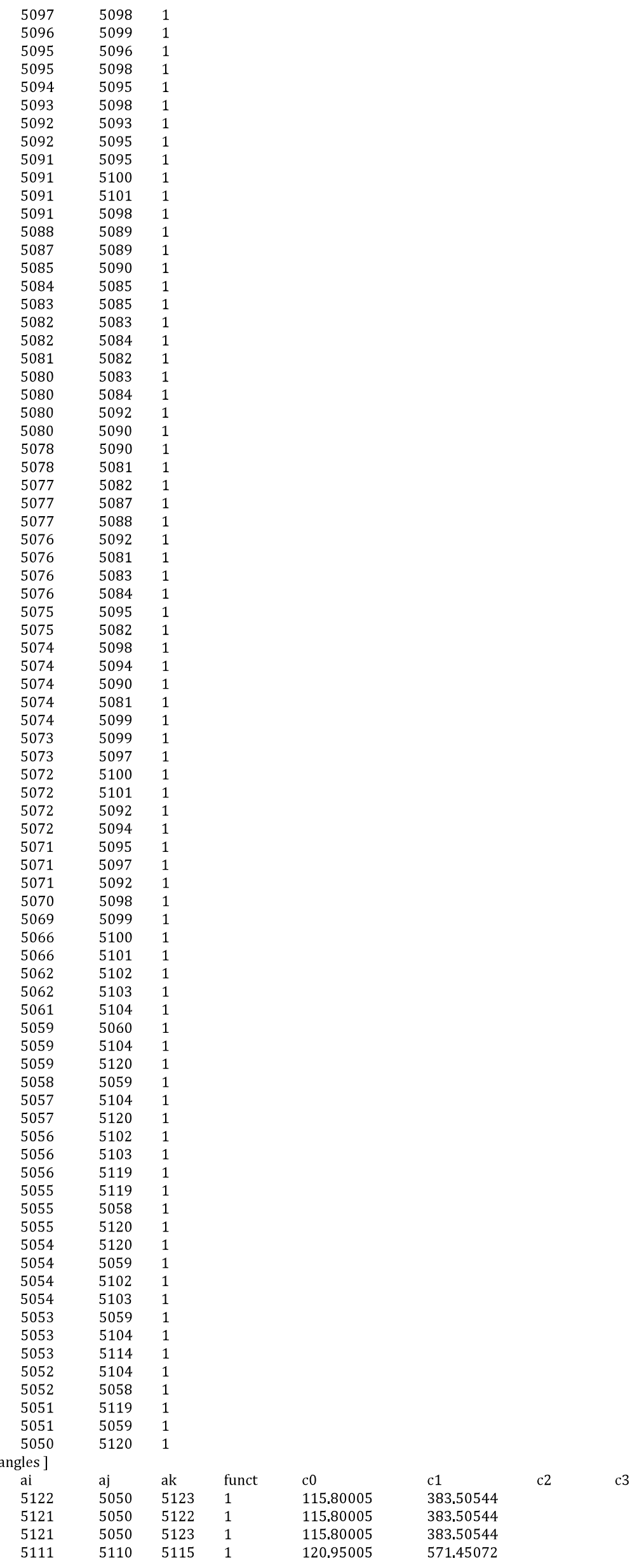




\begin{tabular}{|c|c|c|c|c|c|}
\hline 5111 & 5112 & 5113 & 1 & 104.24004 & 596.80576 \\
\hline 5110 & 5111 & 5112 & 1 & 119.72005 & 581.24128 \\
\hline 5109 & 5110 & 5111 & 1 & 119.72005 & 581.24128 \\
\hline 5109 & 5110 & 5115 & 1 & 118.86005 & 600.48768 \\
\hline 5108 & 5109 & 5110 & 1 & 104.24004 & 596.80576 \\
\hline 5107 & 5106 & 5111 & 1 & 119.72005 & 581.24128 \\
\hline 5107 & 5108 & 5109 & 1 & 125.70005 & 583.83536 \\
\hline 5106 & 5105 & 5113 & 1 & 104.24004 & 596.80576 \\
\hline 5106 & 5107 & 5108 & 1 & 104.24004 & 596.80576 \\
\hline 5106 & 5111 & 5110 & 1 & 120.02005 & 557.47616 \\
\hline 5106 & 5111 & 5112 & 1 & 119.72005 & 581.24128 \\
\hline 5105 & 5106 & 5107 & 1 & 128.74006 & 584.5048 \\
\hline 5105 & 5106 & 5111 & 1 & 119.72005 & 581.24128 \\
\hline 5105 & 5113 & 5112 & 1 & 125.70005 & 583.83536 \\
\hline 5086 & 5085 & 5089 & 1 & 123.05005 & 621.07296 \\
\hline 5078 & 5077 & 5085 & 1 & 117.76005 & 529.94544 \\
\hline 5078 & 5079 & 5080 & 1 & 120.77005 & 531.61904 \\
\hline 5077 & 5078 & 5079 & 1 & 112.88005 & 532.79056 \\
\hline 5077 & 5085 & 5086 & 1 & 112.70005 & 577.97776 \\
\hline 5077 & 5085 & 5089 & 1 & 123.93005 & 578.56352 \\
\hline 5076 & 5075 & 5080 & 1 & 124.68005 & 554.12896 \\
\hline 5076 & 5077 & 5078 & 1 & 126.52005 & 513.29312 \\
\hline 5076 & 5077 & 5085 & 1 & 122.95005 & 526.93296 \\
\hline 5075 & 5074 & 5091 & 1 & 108.51005 & 597.4752 \\
\hline 5075 & 5076 & 5077 & 1 & 120.95005 & 571.45072 \\
\hline 5075 & 5080 & 5079 & 1 & 120.95005 & 571.45072 \\
\hline 5074 & 5073 & 5093 & 1 & 110.19005 & 564.58896 \\
\hline 5074 & 5075 & 5076 & 1 & 118.51505 & 540.957728 \\
\hline 5074 & 5075 & 5080 & 1 & 118.51505 & 540.957728 \\
\hline 5073 & 5072 & 5096 & 1 & 110.19005 & 564.58896 \\
\hline 5073 & 5074 & 5075 & 1 & 111.04005 & 552.45536 \\
\hline 5073 & 5074 & 5091 & 1 & 107.97005 & 569.024 \\
\hline 5072 & 5071 & 5091 & 1 & 107.97005 & 569.024 \\
\hline 5072 & 5073 & 5074 & 1 & 111.51005 & 526.01248 \\
\hline 5072 & 5073 & 5093 & 1 & 110.19005 & 564.58896 \\
\hline 5071 & 5072 & 5073 & 1 & 111.51005 & 526.01248 \\
\hline 5071 & 5072 & 5096 & 1 & 110.19005 & 564.58896 \\
\hline 5071 & 5091 & 5074 & 1 & 112.48005 & 524.6736 \\
\hline 5070 & 5071 & 5072 & 1 & 111.51005 & 526.01248 \\
\hline 5070 & 5071 & 5091 & 1 & 107.97005 & 569.024 \\
\hline 5069 & 5070 & 5071 & 1 & 107.97005 & 569.024 \\
\hline 5068 & 5066 & 5069 & 1 & 115.46005 & 366.9368 \\
\hline 5067 & 5066 & 5068 & 1 & 115.80005 & 383.50544 \\
\hline 5067 & 5066 & 5069 & 1 & 115.46005 & 366.9368 \\
\hline 5066 & 5069 & 5070 & 1 & 119.54005 & 650.36096 \\
\hline 5065 & 5066 & 5067 & 1 & 115.46005 & 366.9368 \\
\hline 5065 & 5066 & 5068 & 1 & 115.46005 & 366.9368 \\
\hline 5065 & 5066 & 5069 & 1 & 101.84004 & 376.56 \\
\hline 5064 & 5062 & 5065 & 1 & 115.46005 & 366.9368 \\
\hline 5063 & 5062 & 5064 & 1 & 115.80005 & 383.50544 \\
\hline 5063 & 5062 & 5065 & 1 & 115.46005 & 366.9368 \\
\hline 5062 & 5065 & 5066 & 1 & 126.25005 & 823.4112 \\
\hline 5061 & 5062 & 5063 & 1 & 115.46005 & 366.9368 \\
\hline 5061 & 5062 & 5064 & 1 & 115.46005 & 366.9368 \\
\hline 5061 & 5062 & 5065 & 1 & 101.84004 & 376.56 \\
\hline 5060 & 5061 & 5062 & 1 & 119.54005 & 650.36096 \\
\hline 5056 & 5055 & 5060 & 1 & 111.51005 & 526.01248 \\
\hline 5055 & 5056 & 5057 & 1 & 110.19005 & 564.58896 \\
\hline 5055 & 5060 & 5061 & 1 & 107.97005 & 569.024 \\
\hline 5054 & 5053 & 5105 & 1 & 115.41005 & 581.1576 \\
\hline 5054 & 5055 & 5056 & 1 & 107.97005 & 569.024 \\
\hline 5054 & 5055 & 5060 & 1 & 107.97005 & 569.024 \\
\hline 5053 & 5052 & 5056 & 1 & 111.51005 & 526.01248 \\
\hline 5053 & 5054 & 5055 & 1 & 112.48005 & 524.6736 \\
\hline 5053 & 5105 & 5106 & 1 & 109.51005 & 568.52192 \\
\hline 5053 & 5105 & 5113 & 1 & 109.51005 & 568.52192 \\
\hline 5052 & 5053 & 5054 & 1 & 107.97005 & 569.024 \\
\hline 5052 & 5053 & 5105 & 1 & 108.80005 & 557.89456 \\
\hline 5052 & 5056 & 5055 & 1 & 111.51005 & 526.01248 \\
\hline 5052 & 5056 & 5057 & 1 & 110.19005 & 564.58896 \\
\hline 5051 & 5050 & 5121 & 1 & 115.46005 & 366.9368 \\
\hline 5051 & 5050 & 5122 & 1 & 115.46005 & 366.9368 \\
\hline 5051 & 5050 & 5123 & 1 & 115.46005 & 366.9368 \\
\hline 5051 & 5052 & 5053 & 1 & 107.97005 & 569.024 \\
\hline 5051 & 5052 & 5056 & 1 & 107.97005 & 569.024 \\
\hline 5050 & 5051 & 5052 & 1 & 119.54005 & 650.36096 \\
\hline 5116 & 5115 & 5117 & 1 & 115.12005 & 335.30576 \\
\hline 5112 & 5113 & 5114 & 1 & 122.92005 & 415.13648 \\
\hline 5110 & 5115 & 5116 & 1 & 116.07005 & 405.17856 \\
\hline 5110 & 5115 & 5117 & 1 & 116.07005 & 405.17856 \\
\hline 5109 & 5108 & 5118 & 1 & 122.92005 & 415.13648 \\
\hline 5107 & 5108 & 5118 & 1 & 122.92005 & 415.13648 \\
\hline 5105 & 5053 & 5119 & 1 & 109.47005 & 417.39584 \\
\hline 5105 & 5113 & 5114 & 1 & 122.92005 & 415.13648 \\
\hline 5102 & 5060 & 5103 & 1 & 108.46005 & 328.36032 \\
\hline 5100 & 5070 & 5101 & 1 & 108.46005 & 328.36032 \\
\hline
\end{tabular}




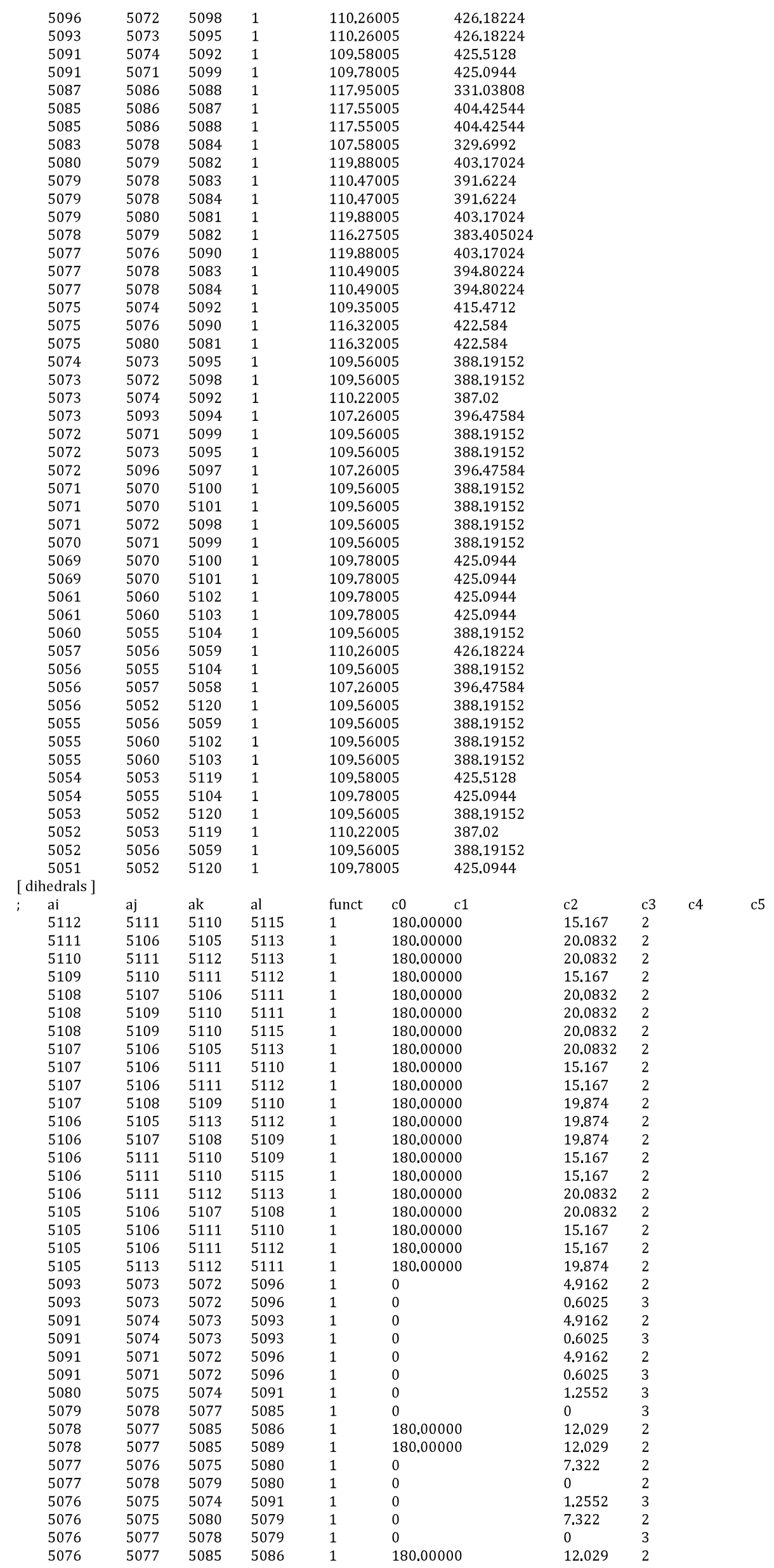




\begin{tabular}{|c|c|c|c|c|c|c|}
\hline 5076 & 5077 & 5085 & 5089 & 1 & 180.00000 & 12.029 \\
\hline 5075 & 5074 & 5073 & 5093 & 1 & 0 & 0.65084 \\
\hline 5075 & 5076 & 5077 & 5078 & 1 & 180.00000 & 2.9288 \\
\hline 5075 & 5076 & 5077 & 5085 & 1 & 180.00000 & 2.9288 \\
\hline 5075 & 5080 & 5079 & 5078 & 1 & 180.00000 & 15.167 \\
\hline 5074 & 5073 & 5072 & 5096 & 1 & 0 & 0.65084 \\
\hline 5074 & 5075 & 5076 & 5077 & 1 & 0 & 7.322 \\
\hline 5074 & 5075 & 5080 & 5079 & 1 & 0 & 7.322 \\
\hline 5073 & 5072 & 5071 & 5091 & 1 & 0 & 0.65084 \\
\hline 5073 & 5074 & 5075 & 5076 & 1 & 0 & 1.2552 \\
\hline 5073 & 5074 & 5075 & 5080 & 1 & 0 & 1.2552 \\
\hline 5072 & 5071 & 5091 & 5074 & 1 & 180.00000 & 0.4184 \\
\hline 5072 & 5071 & 5091 & 5074 & 1 & 0 & 1.60247 \\
\hline 5072 & 5073 & 5074 & 5075 & 1 & 0 & 0.65084 \\
\hline 5072 & 5073 & 5074 & 5091 & 1 & 0 & 0.65084 \\
\hline 5071 & 5072 & 5073 & 5074 & 1 & 180.00000 & 0.8368 \\
\hline 5071 & 5072 & 5073 & 5074 & 1 & 180.00000 & 1.046 \\
\hline 5071 & 5072 & 5073 & 5074 & 1 & 0 & 0.75312 \\
\hline 5071 & 5072 & 5073 & 5093 & 1 & 0 & 0.65084 \\
\hline 5071 & 5091 & 5074 & 5073 & 1 & 180.00000 & 0.4184 \\
\hline 5071 & 5091 & 5074 & 5073 & 1 & 0 & 1.60247 \\
\hline 5071 & 5091 & 5074 & 5075 & 1 & 0 & 1.60387 \\
\hline 5070 & 5071 & 5072 & 5073 & 1 & 180.00000 & 0.8368 \\
\hline 5070 & 5071 & 5072 & 5073 & 1 & 180.00000 & 1.046 \\
\hline 5070 & 5071 & 5072 & 5073 & 1 & 0 & 0.75312 \\
\hline 5070 & 5071 & 5072 & 5096 & 1 & 0 & 0.65084 \\
\hline 5070 & 5071 & 5091 & 5074 & 1 & 180.00000 & 0.4184 \\
\hline 5070 & 5071 & 5091 & 5074 & 1 & 0 & 1.60247 \\
\hline 5069 & 5070 & 5071 & 5072 & 1 & 0 & 0.65084 \\
\hline 5069 & 5070 & 5071 & 5091 & 1 & 0 & 4.9162 \\
\hline 5069 & 5070 & 5071 & 5091 & 1 & 0 & 0.6025 \\
\hline 5068 & 5066 & 5069 & 5070 & 1 & 0 & 3.3472 \\
\hline 5068 & 5066 & 5069 & 5070 & 1 & 0 & 2.3012 \\
\hline 5067 & 5066 & 5069 & 5070 & 1 & 0 & 3.3472 \\
\hline 5067 & 5066 & 5069 & 5070 & 1 & 0 & 2.3012 \\
\hline 5066 & 5069 & 5070 & 5071 & 1 & 180.00000 & 16.5268 \\
\hline 5066 & 5069 & 5070 & 5071 & 1 & 0 & 1.60387 \\
\hline 5065 & 5066 & 5069 & 5070 & 1 & 0 & 5.0208 \\
\hline 5065 & 5066 & 5069 & 5070 & 1 & 0 & 1.046 \\
\hline 5064 & 5062 & 5065 & 5066 & 1 & 0 & 3.3472 \\
\hline 5063 & 5062 & 5065 & 5066 & 1 & 0 & 3.3472 \\
\hline 5062 & 5065 & 5066 & 5067 & 1 & 0 & 3.3472 \\
\hline 5062 & 5065 & 5066 & 5068 & 1 & 0 & 3.3472 \\
\hline 5062 & 5065 & 5066 & 5069 & 1 & 0 & 3.3472 \\
\hline 5061 & 5062 & 5065 & 5066 & 1 & 0 & 3.3472 \\
\hline 5060 & 5061 & 5062 & 5063 & 1 & 0 & 3.3472 \\
\hline 5060 & 5061 & 5062 & 5063 & 1 & 0 & 2.3012 \\
\hline 5060 & 5061 & 5062 & 5064 & 1 & 0 & 3.3472 \\
\hline 5060 & 5061 & 5062 & 5064 & 1 & 0 & 2,3012 \\
\hline 5060 & 5061 & 5062 & 5065 & 1 & 0 & 5.0208 \\
\hline 5060 & 5061 & 5062 & 5065 & 1 & 0 & 1.046 \\
\hline 5057 & 5056 & 5055 & 5060 & 1 & 0 & 0.65084 \\
\hline 5056 & 5055 & 5060 & 5061 & 1 & 0 & 0.65084 \\
\hline 5056 & 5052 & 5053 & 5105 & 1 & 0 & 0.65084 \\
\hline 5055 & 5054 & 5053 & 5105 & 1 & 0 & 1.60387 \\
\hline 5055 & 5060 & 5061 & 5062 & 1 & 180.00000 & 16.5268 \\
\hline 5055 & 5060 & 5061 & 5062 & 1 & 0 & 1.60387 \\
\hline 5054 & 5053 & 5052 & 5056 & 1 & 0 & 0.65084 \\
\hline 5054 & 5053 & 5105 & 5106 & 1 & 0 & 0 \\
\hline 5054 & 5053 & 5105 & 5113 & 1 & 0 & 0 \\
\hline 5054 & 5055 & 5056 & 5057 & 1 & 0 & 4.9162 \\
\hline 5054 & 5055 & 5056 & 5057 & 1 & 0 & 0.6025 \\
\hline 5054 & 5055 & 5060 & 5061 & 1 & 0 & 4.9162 \\
\hline 5054 & 5055 & 5060 & 5061 & 1 & 0 & 0.6025 \\
\hline 5053 & 5052 & 5056 & 5055 & 1 & 180.00000 & 0.8368 \\
\hline 5053 & 5052 & 5056 & 5055 & 1 & 180.00000 & 1.046 \\
\hline 5053 & 5052 & 5056 & 5055 & 1 & 0 & 0.75312 \\
\hline 5053 & 5052 & 5056 & 5057 & 1 & 0 & 0.65084 \\
\hline 5053 & 5054 & 5055 & 5056 & 1 & 180.00000 & 0.4184 \\
\hline 5053 & 5054 & 5055 & 5056 & 1 & 0 & 1.60247 \\
\hline 5053 & 5054 & 5055 & 5060 & 1 & 180.00000 & 0.4184 \\
\hline 5053 & 5054 & 5055 & 5060 & 1 & 0 & 1.60247 \\
\hline 5053 & 5105 & 5106 & 5107 & 1 & 180.00000 & 20.0832 \\
\hline 5053 & 5105 & 5106 & 5111 & 1 & 180.00000 & 20.0832 \\
\hline 5053 & 5105 & 5113 & 5112 & 1 & 180.00000 & 19.874 \\
\hline 5052 & 5051 & 5050 & 5121 & 1 & 0 & 3.3472 \\
\hline 5052 & 5051 & 5050 & 5121 & 1 & 0 & 2,3012 \\
\hline 5052 & 5051 & 5050 & 5122 & 1 & 0 & 3.3472 \\
\hline 5052 & 5051 & 5050 & 5122 & 1 & 0 & 2.3012 \\
\hline 5052 & 5051 & 5050 & 5123 & 1 & 0 & 3.3472 \\
\hline 5052 & 5051 & 5050 & 5123 & 1 & 0 & 2.3012 \\
\hline 5052 & 5053 & 5054 & 5055 & 1 & 180.00000 & 0.4184 \\
\hline 5052 & 5053 & 5054 & 5055 & 1 & 0 & 1.60247 \\
\hline 5052 & 5053 & 5105 & 5106 & 1 & 0 & 0 \\
\hline 5052 & 5053 & 5105 & 5113 & 1 & 0 & 0 \\
\hline 5052 & 5056 & 5055 & 5054 & 1 & 0 & 0.65084 \\
\hline
\end{tabular}




\begin{tabular}{|c|c|c|c|c|c|c|}
\hline 5052 & 5056 & 5055 & 5060 & 1 & 180.00000 & 0.8368 \\
\hline 5052 & 5056 & 5055 & 5060 & 1 & 180.00000 & 1.046 \\
\hline 5052 & 5056 & 5055 & 5060 & 1 & 0 & 0.75312 \\
\hline 5051 & 5052 & 5053 & 5054 & 1 & 0 & 4.9162 \\
\hline 5051 & 5052 & 5053 & 5054 & 1 & 0 & 0.6025 \\
\hline 5051 & 5052 & 5053 & 5105 & 1 & 0 & 0.65084 \\
\hline 5051 & 5052 & 5056 & 5055 & 1 & 0 & 0.65084 \\
\hline 5051 & 5052 & 5056 & 5057 & 1 & 0 & 4.9162 \\
\hline 5051 & 5052 & 5056 & 5057 & 1 & 0 & 0.6025 \\
\hline 5050 & 5051 & 5052 & 5053 & 1 & 180.00000 & 16.5268 \\
\hline 5050 & 5051 & 5052 & 5053 & 1 & 0 & 1.60387 \\
\hline 5050 & 5051 & 5052 & 5056 & 1 & 180.00000 & 16.5268 \\
\hline 5050 & 5051 & 5052 & 5056 & 1 & 0 & 1.60387 \\
\hline 5110 & 5106 & 5111 & 5112 & 4 & 180.00000 & 4.6024 \\
\hline 5111 & 5115 & 5110 & 5109 & 4 & 180.00000 & 4.6024 \\
\hline 5111 & 5105 & 5106 & 5107 & 4 & 180.00000 & 43.932 \\
\hline 5106 & 5113 & 5105 & 5053 & 4 & 180.00000 & 4.6024 \\
\hline 5077 & 5086 & 5085 & 5089 & 4 & 180.00000 & 43.932 \\
\hline 5085 & 5076 & 5077 & 5078 & 4 & 180.00000 & 4.6024 \\
\hline 5119 & 5053 & 5052 & 5120 & 1 & 0 & 0.65084 \\
\hline 5113 & 5105 & 5053 & 5119 & 1 & 0 & 0 \\
\hline 5111 & 5110 & 5115 & 5116 & 1 & 180.00000 & 4.3932 \\
\hline 5111 & 5110 & 5115 & 5117 & 1 & 180.00000 & 4.3932 \\
\hline 5111 & 5112 & 5113 & 5114 & 1 & 180.00000 & 19.874 \\
\hline 5110 & 5109 & 5108 & 5118 & 1 & 180.00000 & 19.874 \\
\hline 5109 & 5110 & 5115 & 5116 & 1 & 180.00000 & 4.3932 \\
\hline 5109 & 5110 & 5115 & 5117 & 1 & 180.00000 & 4.3932 \\
\hline 5106 & 5105 & 5053 & 5119 & 1 & 0 & 0 \\
\hline 5106 & 5105 & 5113 & 5114 & 1 & 180.00000 & 19.874 \\
\hline 5106 & 5107 & 5108 & 5118 & 1 & 180.00000 & 19.874 \\
\hline 5105 & 5053 & 5052 & 5120 & 1 & 0 & 0.65084 \\
\hline 5103 & 5060 & 5055 & 5104 & 1 & 0 & 0.65084 \\
\hline 5102 & 5060 & 5055 & 5104 & 1 & 0 & 0.65084 \\
\hline 5099 & 5071 & 5070 & 5100 & 1 & 0 & 0.65084 \\
\hline 5099 & 5071 & 5070 & 5101 & 1 & 0 & 0.65084 \\
\hline 5098 & 5072 & 5071 & 5099 & 1 & 0 & 0.65084 \\
\hline 5097 & 5096 & 5072 & 5098 & 1 & 0 & 0.69733 \\
\hline 5096 & 5072 & 5071 & 5099 & 1 & 0 & 1.046 \\
\hline 5096 & 5072 & 5071 & 5099 & 1 & 0 & 0 \\
\hline 5095 & 5073 & 5072 & 5096 & 1 & 0 & 1.046 \\
\hline 5095 & 5073 & 5072 & 5096 & 1 & 0 & 0 \\
\hline 5095 & 5073 & 5072 & 5098 & 1 & 0 & 0.65084 \\
\hline 5094 & 5093 & 5073 & 5095 & 1 & 0 & 0.69733 \\
\hline 5093 & 5073 & 5072 & 5098 & 1 & 0 & 1.046 \\
\hline 5093 & 5073 & 5072 & 5098 & 1 & 0 & 0 \\
\hline 5092 & 5074 & 5073 & 5093 & 1 & 0 & 0.65084 \\
\hline 5092 & 5074 & 5073 & 5095 & 1 & 0 & 0.65084 \\
\hline 5091 & 5074 & 5073 & 5095 & 1 & 0 & 1.046 \\
\hline 5091 & 5074 & 5073 & 5095 & 1 & 0 & 0 \\
\hline 5091 & 5071 & 5070 & 5100 & 1 & 0 & 1.046 \\
\hline 5091 & 5071 & 5070 & 5100 & 1 & 0 & 0 \\
\hline 5091 & 5071 & 5070 & 5101 & 1 & 0 & 1.046 \\
\hline 5091 & 5071 & 5070 & 5101 & 1 & 0 & 0 \\
\hline 5091 & 5071 & 5072 & 5098 & 1 & 0 & 1.046 \\
\hline 5091 & 5071 & 5072 & 5098 & 1 & 0 & 0 \\
\hline 5088 & 5086 & 5085 & 5089 & 1 & 0 & 8.368 \\
\hline 5088 & 5086 & 5085 & 5089 & 1 & 180.00000 & 10.46 \\
\hline 5087 & 5086 & 5085 & 5089 & 1 & 0 & 8.368 \\
\hline 5087 & 5086 & 5085 & 5089 & 1 & 180.00000 & 10.46 \\
\hline 5085 & 5077 & 5076 & 5090 & 1 & 180.00000 & 2.9288 \\
\hline 5084 & 5078 & 5077 & 5085 & 1 & 0 & 0 \\
\hline 5083 & 5078 & 5077 & 5085 & 1 & 0 & 0 \\
\hline 5082 & 5079 & 5078 & 5083 & 1 & 0 & 0 \\
\hline 5082 & 5079 & 5078 & 5084 & 1 & 0 & 0 \\
\hline 5081 & 5080 & 5079 & 5082 & 1 & 180.00000 & 15.167 \\
\hline 5080 & 5079 & 5078 & 5083 & 1 & 0 & 0 \\
\hline 5080 & 5079 & 5078 & 5084 & 1 & 0 & 0 \\
\hline 5080 & 5075 & 5074 & 5092 & 1 & 0 & 1.2552 \\
\hline 5080 & 5075 & 5076 & 5090 & 1 & 0 & 7.322 \\
\hline 5078 & 5077 & 5076 & 5090 & 1 & 180.00000 & 2.9288 \\
\hline 5078 & 5079 & 5080 & 5081 & 1 & 180.00000 & 15.167 \\
\hline 5077 & 5078 & 5079 & 5082 & 1 & 0 & 0 \\
\hline 5077 & 5085 & 5086 & 5087 & 1 & 180.00000 & 10.46 \\
\hline 5077 & 5085 & 5086 & 5088 & 1 & 180.00000 & 10.46 \\
\hline 5076 & 5075 & 5074 & 5092 & 1 & 0 & 1.2552 \\
\hline 5076 & 5075 & 5080 & 5081 & 1 & 0 & 7.322 \\
\hline 5076 & 5077 & 5078 & 5083 & 1 & 0 & 0 \\
\hline 5076 & 5077 & 5078 & 5084 & 1 & 0 & 0 \\
\hline 5075 & 5074 & 5073 & 5095 & 1 & 0 & 0.65084 \\
\hline 5075 & 5080 & 5079 & 5082 & 1 & 180.00000 & 15.167 \\
\hline 5074 & 5073 & 5072 & 5098 & 1 & 0 & 0.65084 \\
\hline 5074 & 5073 & 5093 & 5094 & 1 & 0 & 1.046 \\
\hline 5074 & 5073 & 5093 & 5094 & 1 & 0 & 0.66944 \\
\hline 5074 & 5075 & 5076 & 5090 & 1 & 0 & 7.322 \\
\hline 5074 & 5075 & 5080 & 5081 & 1 & 0 & 7.322 \\
\hline 5074 & 5091 & 5071 & 5099 & 1 & 0 & 1.60387 \\
\hline
\end{tabular}




\begin{tabular}{|c|c|c|c|c|c|c|}
\hline 5073 & 5072 & 5071 & 5099 & 1 & 0 & 0.65084 \\
\hline 5073 & 5072 & 5096 & 5097 & 1 & 0 & 1.046 \\
\hline 5073 & 5072 & 5096 & 5097 & 1 & 0 & 0.66944 \\
\hline 5072 & 5071 & 5070 & 5100 & 1 & 0 & 0.65084 \\
\hline 5072 & 5071 & 5070 & 5101 & 1 & 0 & 0.65084 \\
\hline 5072 & 5073 & 5074 & 5092 & 1 & 0 & 0.65084 \\
\hline 5072 & 5073 & 5093 & 5094 & 1 & 0 & 1.046 \\
\hline 5072 & 5073 & 5093 & 5094 & 1 & 0 & 0.66944 \\
\hline 5071 & 5072 & 5073 & 5095 & 1 & 0 & 0.65084 \\
\hline 5071 & 5072 & 5096 & 5097 & 1 & 0 & 1.046 \\
\hline 5071 & 5072 & 5096 & 5097 & 1 & 0 & 0.66944 \\
\hline 5071 & 5091 & 5074 & 5092 & 1 & 0 & 1.60387 \\
\hline 5070 & 5071 & 5072 & 5098 & 1 & 0 & 0.65084 \\
\hline 5069 & 5070 & 5071 & 5099 & 1 & 0 & 1.046 \\
\hline 5069 & 5070 & 5071 & 5099 & 1 & 0 & 0 \\
\hline 5066 & 5069 & 5070 & 5100 & 1 & 0 & 1.60387 \\
\hline 5066 & 5069 & 5070 & 5101 & 1 & 0 & 1.60387 \\
\hline 5062 & 5061 & 5060 & 5102 & 1 & 0 & 1.60387 \\
\hline 5062 & 5061 & 5060 & 5103 & 1 & 0 & 1.60387 \\
\hline 5061 & 5060 & 5055 & 5104 & 1 & 0 & 1.046 \\
\hline 5061 & 5060 & 5055 & 5104 & 1 & 0 & 0 \\
\hline 5059 & 5056 & 5055 & 5060 & 1 & 0 & 0.65084 \\
\hline 5059 & 5056 & 5055 & 5104 & 1 & 0 & 0.65084 \\
\hline 5059 & 5056 & 5052 & 5120 & 1 & 0 & 0.65084 \\
\hline 5058 & 5057 & 5056 & 5059 & 1 & 0 & 0.69733 \\
\hline 5057 & 5056 & 5055 & 5104 & 1 & 0 & 1.046 \\
\hline 5057 & 5056 & 5055 & 5104 & 1 & 0 & 0 \\
\hline 5057 & 5056 & 5052 & 5120 & 1 & 0 & 1.046 \\
\hline 5057 & 5056 & 5052 & 5120 & 1 & 0 & 0 \\
\hline 5056 & 5055 & 5060 & 5102 & 1 & 0 & 0.65084 \\
\hline 5056 & 5055 & 5060 & 5103 & 1 & 0 & 0.65084 \\
\hline 5056 & 5052 & 5053 & 5119 & 1 & 0 & 0.65084 \\
\hline 5055 & 5054 & 5053 & 5119 & 1 & 0 & 1.60387 \\
\hline 5055 & 5056 & 5057 & 5058 & 1 & 0 & 1.046 \\
\hline 5055 & 5056 & 5057 & 5058 & 1 & 0 & 0.66944 \\
\hline 5055 & 5056 & 5052 & 5120 & 1 & 0 & 0.65084 \\
\hline 5054 & 5053 & 5052 & 5120 & 1 & 0 & 1.046 \\
\hline 5054 & 5053 & 5052 & 5120 & 1 & 0 & 0 \\
\hline 5054 & 5055 & 5056 & 5059 & 1 & 0 & 1.046 \\
\hline 5054 & 5055 & 5056 & 5059 & 1 & 0 & 0 \\
\hline 5054 & 5055 & 5060 & 5102 & 1 & 0 & 1.046 \\
\hline 5054 & 5055 & 5060 & 5102 & 1 & 0 & 0 \\
\hline 5054 & 5055 & 5060 & 5103 & 1 & 0 & 1.046 \\
\hline 5054 & 5055 & 5060 & 5103 & 1 & 0 & 0 \\
\hline 5053 & 5052 & 5056 & 5059 & 1 & 0 & 0.65084 \\
\hline 5053 & 5054 & 5055 & 5104 & 1 & 0 & 1.60387 \\
\hline 5053 & 5105 & 5113 & 5114 & 1 & 180.00000 & 19.874 \\
\hline 5052 & 5056 & 5055 & 5104 & 1 & 0 & 0.65084 \\
\hline 5052 & 5056 & 5057 & 5058 & 1 & 0 & 1.046 \\
\hline 5052 & 5056 & 5057 & 5058 & 1 & 0 & 0.66944 \\
\hline 5051 & 5052 & 5053 & 5119 & 1 & 0 & 0.65084 \\
\hline 5051 & 5052 & 5056 & 5059 & 1 & 0 & 1.046 \\
\hline 5051 & 5052 & 5056 & 5059 & 1 & 0 & 0 \\
\hline 5050 & 5051 & 5052 & 5120 & 1 & 0 & 1.60387 \\
\hline 5110 & 5116 & 5115 & 5117 & 4 & $180.000 \quad 08$ & 4.6024 \\
\hline 5114 & 5105 & 5113 & 5112 & 4 & 180.00000 & 43.932 \\
\hline 5118 & 5107 & 5108 & 5109 & 4 & 180.00000 & 43.932 \\
\hline 5085 & 5087 & 5086 & 5088 & 4 & 180.00000 & 4.6024 \\
\hline 5079 & 5081 & 5080 & 5075 & 4 & 180.00000 & 4,6024 \\
\hline 5080 & 5078 & 5079 & 5082 & 4 & 180.00000 & 4.6024 \\
\hline 5077 & 5090 & 5076 & 5075 & 4 & 180.00000 & 4.6024 \\
\hline
\end{tabular}

PChlide parameters from ANTECHAMBER and DFT modelling: [atoms ]

\begin{tabular}{|c|c|c|c|c|c|c|c|c|c|c|}
\hline $\mathrm{nr}$ & type & resnr & residue & atom & cgnr & charge & mass & typeB & chargeB & massB \\
\hline 5124 & $\mathrm{~N}$ & 1 & $\mathrm{PCH}$ & $\mathrm{N} 1$ & 1 & -0.49953 & 14.01 & ; & qtot & -0.49953 \\
\hline 5125 & $\mathrm{CC}$ & 1 & $\mathrm{PCH}$ & C16 & 1 & 0.380229 & 12.01 & ; & qtot & -0.119301 \\
\hline 5126 & $\mathrm{C}^{*}$ & 1 & $\mathrm{PCH}$ & C15 & 1 & -0.212583 & 12.01 & ; & qtot & -0.331884 \\
\hline 5127 & $\mathrm{C}^{*}$ & 1 & $\mathrm{PCH}$ & C14 & 1 & 0.077312 & 12.01 & ; & qtot & -0.254572 \\
\hline 5128 & CT & 1 & $\mathrm{PCH}$ & C29 & 1 & -0.16082 & 12.01 & ; & qtot & -0.415392 \\
\hline 5129 & $\mathrm{HC}$ & 1 & $\mathrm{PCH}$ & $\mathrm{H} 23$ & 1 & 0.036552 & 1.008 & ; & qtot & -0.37884 \\
\hline 5130 & $\mathrm{HC}$ & 1 & $\mathrm{PCH}$ & $\mathrm{H} 24$ & 1 & 0.036552 & 1.008 & ; & qtot & -0.342288 \\
\hline 5131 & $\mathrm{HC}$ & 1 & $\mathrm{PCH}$ & $\mathrm{H} 25$ & 1 & 0.036552 & 1.008 & ; & qtot & -0.305736 \\
\hline 5132 & $C D$ & 1 & $\mathrm{PCH}$ & C27 & 1 & 0.026312 & 12.01 & ; & qtot & -0.279424 \\
\hline 5133 & CM & 1 & $\mathrm{PCH}$ & C28 & 1 & -0.576324 & 12.01 & ; & qtot & -0.855748 \\
\hline 5134 & HA & 1 & $\mathrm{PCH}$ & $\mathrm{H} 21$ & 1 & 0.187605 & 1.008 & ; & qtot & -0.668143 \\
\hline 5135 & HA & 1 & $\mathrm{PCH}$ & $\mathrm{H} 22$ & 1 & 0.187605 & 1.008 & ; & qtot & -0.480538 \\
\hline 5136 & $\mathrm{HA}$ & 1 & $\mathrm{PCH}$ & $\mathrm{H} 20$ & 1 & 0.106657 & 1.008 & ; & qtot & -0.373881 \\
\hline 5137 & $C D$ & 1 & $\mathrm{PCH}$ & C17 & 1 & -0.453788 & 12.01 & ; & qtot & -0.827669 \\
\hline 5138 & CC & 1 & $\mathrm{PCH}$ & $\mathrm{C} 1$ & 1 & 0.256454 & 12.01 & ; & qtot & -0.571215 \\
\hline 5139 & $\mathrm{~N}$ & 1 & $\mathrm{PCH}$ & $\mathrm{N} 2$ & 1 & -0.51625 & 14.01 & ; & qtot & -1.087465 \\
\hline 5140 & CC & 1 & $\mathrm{PCH}$ & C4 & 1 & 0.392357 & 12.01 & ; & qtot & -0.695108 \\
\hline 5141 & $\mathrm{C}^{*}$ & 1 & $\mathrm{PCH}$ & C3 & 1 & -0.393595 & 12.01 & ; & qtot & -1.088703 \\
\hline 5142 & $\mathrm{C}^{*}$ & 1 & $\mathrm{PCH}$ & $\mathrm{C} 2$ & 1 & 0.110842 & 12.01 & ; & qtot & -0.977861 \\
\hline 5143 & CT & 1 & $\mathrm{PCH}$ & C26 & 1 & -0.197749 & 12.01 & ; & qtot & -1.17561 \\
\hline 5144 & $\mathrm{HC}$ & 1 & $\mathrm{PCH}$ & $\mathrm{H} 17$ & 1 & 0.049179 & 1.008 & ; & qtot & -1.126431 \\
\hline
\end{tabular}




\begin{tabular}{|c|c|c|c|c|c|c|c|c|c|c|}
\hline 5145 & $\mathrm{HC}$ & 1 & $\mathrm{PCH}$ & H18 & 1 & 0.049179 & 1.008 & ; & qtot & -1.077252 \\
\hline 5146 & $\mathrm{HC}$ & 1 & $\mathrm{PCH}$ & H19 & 1 & 0.049179 & 1.008 & ; & qtot & -1.028073 \\
\hline 5147 & CT & 1 & $\mathrm{PCH}$ & $\mathrm{C} 24$ & 1 & 0.317254 & 12.01 & ; & qtot & -0.710819 \\
\hline 5148 & CT & 1 & $\mathrm{PCH}$ & $\mathrm{C} 25$ & 1 & -0.190886 & 12.01 & ; & qtot & -0.901705 \\
\hline 5149 & $\mathrm{HC}$ & 1 & $\mathrm{PCH}$ & H14 & 1 & 0.033443 & 1.008 & ; & qtot & -0.868262 \\
\hline 5150 & $\mathrm{HC}$ & 1 & $\mathrm{PCH}$ & H15 & 1 & 0.033443 & 1.008 & ; & qtot & -0.834819 \\
\hline 5151 & $\mathrm{HC}$ & 1 & $\mathrm{PCH}$ & H16 & 1 & 0.033443 & 1.008 & ; & qtot & -0.801376 \\
\hline 5152 & $\mathrm{HC}$ & 1 & $\mathrm{PCH}$ & $\mathrm{H} 12$ & 1 & -0.067431 & 1.008 & ; & qtot & -0.868807 \\
\hline 5153 & $\mathrm{HC}$ & 1 & $\mathrm{PCH}$ & $\mathrm{H} 13$ & 1 & -0.067431 & 1.008 & ; & qtot & -0.936238 \\
\hline 5154 & CD & 1 & $\mathrm{PCH}$ & C18 & 1 & -0.419627 & 12.01 & ; & qtot & -1.355865 \\
\hline 5155 & CC & 1 & $\mathrm{PCH}$ & $\mathrm{C} 5$ & 1 & 0.263348 & 12.01 & ; & qtot & -1.092517 \\
\hline 5156 & $\mathrm{~N}$ & 1 & $\mathrm{PCH}$ & N3 & 1 & -0.47848 & 14.01 & ; & qtot & -1.570997 \\
\hline 5157 & $\mathrm{CC}$ & 1 & $\mathrm{PCH}$ & $\mathrm{C} 8$ & 1 & 0.180586 & 12.01 & ; & qtot & -1.390411 \\
\hline 5158 & $\mathrm{C}^{*}$ & 1 & $\mathrm{PCH}$ & $\mathrm{C} 7$ & 1 & -0.362807 & 12.01 & ; & qtot & -1.753218 \\
\hline 5159 & $\mathrm{C}^{*}$ & 1 & $\mathrm{PCH}$ & $\mathrm{C} 6$ & 1 & 0.068786 & 12.01 & ; & qtot & -1.684432 \\
\hline 5160 & CT & 1 & $\mathrm{PCH}$ & $\mathrm{C} 23$ & 1 & -0.261748 & 12.01 & ; & qtot & -1.94618 \\
\hline 5161 & $\mathrm{HC}$ & 1 & $\mathrm{PCH}$ & H9 & 1 & 0.068421 & 1.008 & ; & qtot & -1.877759 \\
\hline 5162 & $\mathrm{HC}$ & 1 & $\mathrm{PCH}$ & $\mathrm{H} 10$ & 1 & 0.068421 & 1.008 & ; & qtot & -1.809338 \\
\hline 5163 & $\mathrm{HC}$ & 1 & $\mathrm{PCH}$ & H11 & 1 & 0.068421 & 1.008 & ; & qtot & -1.740917 \\
\hline 5164 & $\mathrm{C}$ & 1 & $\mathrm{PCH}$ & C30 & 1 & 0.800656 & 12.01 & ; & qtot & -0.940261 \\
\hline 5165 & CT & 1 & $\mathrm{PCH}$ & C31 & 1 & -0.760914 & 12.01 & ; & qtot & -1.701175 \\
\hline 5166 & $C D$ & 1 & $\mathrm{PCH}$ & C19 & 1 & 0.169165 & 12.01 & ; & qtot & -1.53201 \\
\hline 5167 & $\mathrm{CC}$ & 1 & $\mathrm{PCH}$ & C9 & 1 & 0.170678 & 12.01 & ; & qtot & $-1,361332$ \\
\hline 5168 & $\mathrm{~N}$ & 1 & $\mathrm{PCH}$ & N4 & 1 & -0.58267 & 14.01 & ; & qtot & -1.944002 \\
\hline 5169 & $\mathrm{CC}$ & 1 & $\mathrm{PCH}$ & C12 & 1 & 0.395672 & 12.01 & ; & qtot & -1.54833 \\
\hline 5170 & $\mathrm{C}^{*}$ & 1 & $\mathrm{PCH}$ & C11 & 1 & -0.001354 & 12.01 & ; & qtot & -1.549684 \\
\hline 5171 & $\mathrm{C}^{*}$ & 1 & $\mathrm{PCH}$ & C10 & 1 & -0.112775 & 12.01 & ; & qtot & -1.662459 \\
\hline 5172 & CT & 1 & $\mathrm{PCH}$ & $\mathrm{C} 22$ & 1 & -0.037798 & 12.01 & ; & qtot & -1.700257 \\
\hline 5173 & CT & 1 & $\mathrm{PCH}$ & C33 & 1 & -0.064751 & 12.01 & ; & qtot & -1.765008 \\
\hline 5174 & C & 1 & $\mathrm{PCH}$ & C34 & 1 & 0.836425 & 12.01 & ; & qtot & -0.928583 \\
\hline 5175 & $\mathrm{O} 2$ & 1 & $\mathrm{PCH}$ & $\mathrm{O} 2$ & 1 & -0.826616 & 16 & ; & qtot & -1.755199 \\
\hline 5176 & $\mathrm{O} 2$ & 1 & $\mathrm{PCH}$ & $\mathrm{O} 3$ & 1 & -0.826616 & 16 & ; & qtot & -2.581815 \\
\hline 5177 & $\mathrm{HC}$ & 1 & $\mathrm{PCH}$ & $\mathrm{H} 27$ & 1 & 0.023741 & 1.008 & ; & qtot & -2.558074 \\
\hline 5178 & $\mathrm{HC}$ & 1 & $\mathrm{PCH}$ & $\mathrm{H} 28$ & 1 & 0.023741 & 1.008 & ; & qtot & -2.534333 \\
\hline 5179 & $\mathrm{HC}$ & 1 & $\mathrm{PCH}$ & H7 & 1 & 0.035214 & 1.008 & ; & qtot & -2.499119 \\
\hline 5180 & $\mathrm{HC}$ & 1 & $\mathrm{PCH}$ & H8 & 1 & 0.035214 & 1.008 & ; & qtot & -2.463905 \\
\hline 5181 & CT & 1 & $\mathrm{PCH}$ & $\mathrm{C} 21$ & 1 & -0.451486 & 12.01 & ; & qtot & -2.915391 \\
\hline 5182 & $\mathrm{HC}$ & 1 & $\mathrm{PCH}$ & $\mathrm{H} 4$ & 1 & 0.120501 & 1.008 & ; & qtot & -2.79489 \\
\hline 5183 & $\mathrm{HC}$ & 1 & $\mathrm{PCH}$ & H5 & 1 & 0.120501 & 1.008 & ; & qtot & -2.674389 \\
\hline 5184 & $\mathrm{HC}$ & 1 & PCH & $\mathrm{H} 6$ & 1 & 0.120501 & 1.008 & ; & qtot & -2.553888 \\
\hline 5185 & $C D$ & 1 & PCH & $\mathrm{C} 20$ & 1 & -0.407613 & 12.01 & ; & qtot & -2.961501 \\
\hline 5186 & HA & 1 & $\mathrm{PCH}$ & H3 & 1 & 0.147682 & 1.008 & ; & qtot & -2.813819 \\
\hline 5187 & C & 1 & PCH & C32 & 1 & 0.989123 & 12.01 & ; & qtot & -1.824696 \\
\hline 5188 & os & 1 & $\mathrm{PCH}$ & 04 & 1 & -0.438966 & 16 & ; & qtot & -2.263662 \\
\hline 5189 & CT & 1 & PCH & C35 & 1 & 0.028714 & 12.01 & ; & qtot & -2.234948 \\
\hline 5190 & $\mathrm{H} 1$ & 1 & $\mathrm{PCH}$ & $\mathrm{H} 29$ & 1 & 0.061439 & 1.008 & ; & qtot & -2.173509 \\
\hline 5191 & H1 & 1 & $\mathrm{PCH}$ & $\mathrm{H} 30$ & 1 & 0.061439 & 1.008 & ; & qtot & -2.11207 \\
\hline 5192 & $\mathrm{H} 1$ & 1 & $\mathrm{PCH}$ & H31 & 1 & 0.061439 & 1.008 & ; & qtot & -2.050631 \\
\hline 5193 & 0 & 1 & PCH & 05 & 1 & -0.593362 & 16 & ; & qtot & $-2,643993$ \\
\hline 5194 & $\mathrm{HC}$ & 1 & $\mathrm{PCH}$ & $\mathrm{H} 26$ & 1 & 0.247358 & 1.008 & ; & qtot & -2.396635 \\
\hline 5195 & 0 & 1 & $\mathrm{PCH}$ & 01 & 1 & -0.644309 & 16 & ; & qtot & $-3,040944$ \\
\hline 5196 & HA & 1 & $\mathrm{PCH}$ & $\mathrm{H} 2$ & 1 & 0.178963 & 1.008 & ; & qtot & -2.861981 \\
\hline 5197 & HA & 1 & $\mathrm{PCH}$ & $\mathrm{H} 1$ & 1 & 0.145857 & 1.008 & ; & qtot & -2.716124 \\
\hline 5198 & CC & 1 & $\mathrm{PCH}$ & C13 & 1 & 0.216127 & 12.01 & ; & qtot & -2.499997 \\
\hline 5199 & MG & 1 & $\mathrm{PCH}$ & MG & 1 & 1.5 & 45.561 & ; & qtot & -0.999997 \\
\hline \multicolumn{11}{|l|}{ [ bonds ] } \\
\hline ; ai & aj & funct & c0 & c1 & c2 & c3 & & & & \\
\hline 5193 & 5189 & 1 & 0.1432 & 2582 & & & & & & \\
\hline 5187 & 5188 & 1 & 0.1358 & 3270 & & & & & & \\
\hline 5187 & 5193 & 1 & 0.1432 & 2582 & & & & & & \\
\hline 5185 & 5198 & 1 & 0.1428 & 3512 & & & & & & \\
\hline 5174 & 5175 & 1 & 0.1218 & 5336 & & & & & & \\
\hline 5174 & 5176 & 1 & 0.1218 & 5336 & & & & & & \\
\hline 5173 & 5174 & 1 & 0.1524 & 2619 & & & & & & \\
\hline 5172 & 5173 & 1 & 0.1538 & 2517 & & & & & & \\
\hline 5171 & 5172 & 1 & 0.1502 & 2801 & & & & & & \\
\hline 5170 & 5171 & 1 & 0.1428 & 3512 & & & & & & \\
\hline 5170 & 5181 & 1 & 0.1502 & 2801 & & & & & & \\
\hline 5169 & 5170 & 1 & 0.1428 & 3512 & & & & & & \\
\hline 5169 & 5185 & 1 & 0.1428 & 3512 & & & & & & \\
\hline 5168 & 5169 & 1 & 0.1369 & 3691 & & & & & & \\
\hline 5167 & 5168 & 1 & 0.1369 & 3691 & & & & & & \\
\hline 5167 & 5171 & 1 & 0.1428 & 3512 & & & & & & \\
\hline 5166 & 5167 & 1 & 0.1428 & 3512 & & & & & & \\
\hline 5165 & 5166 & 1 & 0.1502 & 2801 & & & & & & \\
\hline 5165 & 5187 & 1 & 0.1524 & 2619 & & & & & & \\
\hline 5164 & 5165 & 1 & 0.1524 & 2619 & & & & & & \\
\hline 5164 & 5195 & 1 & 0.1218 & 5336 & & & & & & \\
\hline 5159 & 5160 & 1 & 0.1502 & 2801 & & & & & & \\
\hline 5158 & 5159 & 1 & 0.1428 & 3512 & & & & & & \\
\hline 5158 & 5164 & 1 & 0.1468 & 3104 & & & & & & \\
\hline 5157 & 5158 & 1 & 0.1428 & 3512 & & & & & & \\
\hline 5157 & 5166 & 1 & 0.1428 & 3512 & & & & & & \\
\hline 5156 & 5157 & 1 & 0.1369 & 3691 & & & & & & \\
\hline 5155 & 5156 & 1 & 0.1369 & 3691 & & & & & & \\
\hline 5155 & 5159 & 1 & 0.1428 & 3512 & & & & & & \\
\hline
\end{tabular}




\begin{tabular}{|c|c|c|c|c|}
\hline 5154 & 5155 & 1 & 0.1428 & 351288.64 \\
\hline 5147 & 5148 & 1 & 0.1538 & 251793.12 \\
\hline 5142 & 5143 & 1 & 0.1502 & 280160.64 \\
\hline 5141 & 5142 & 1 & 0.1428 & 351288.64 \\
\hline 5141 & 5147 & 1 & 0.1502 & 280160.64 \\
\hline 5140 & 5141 & 1 & 0.1428 & 351288.64 \\
\hline 5140 & 5154 & 1 & 0.1428 & 351288.64 \\
\hline 5139 & 5140 & 1 & 0.1369 & 369112.48 \\
\hline 5138 & 5139 & 1 & 0.1369 & 369112.48 \\
\hline 5138 & 5142 & 1 & 0.1428 & 351288.64 \\
\hline 5137 & 5138 & 1 & 0.1428 & 351288.64 \\
\hline 5132 & 5133 & 1 & 0.1456 & 322251.68 \\
\hline 5127 & 5128 & 1 & 0.1502 & 280160.64 \\
\hline 5127 & 5198 & 1 & 0.1428 & 351288.64 \\
\hline 5126 & 5127 & 1 & 0.1428 & 351288.64 \\
\hline 5126 & 5132 & 1 & 0.1428 & 351288.64 \\
\hline 5125 & 5126 & 1 & 0.1428 & 351288.64 \\
\hline 5125 & 5137 & 1 & 0.1428 & 351288.64 \\
\hline 5124 & 5125 & 1 & 0.1369 & 369112.48 \\
\hline 5124 & 5198 & 1 & 0.1369 & 369112.48 \\
\hline 5189 & 5190 & 1 & 0.1097 & 276646.08 \\
\hline 5189 & 5191 & 1 & 0.1097 & 276646.08 \\
\hline 5189 & 5192 & 1 & 0.1097 & 276646.08 \\
\hline 5185 & 5186 & 1 & 0.1084 & 292126.88 \\
\hline 5181 & 5182 & 1 & 0.1097 & 276646.08 \\
\hline 5181 & 5183 & 1 & 0.1097 & 276646.08 \\
\hline 5181 & 5184 & 1 & 0.1097 & 276646.08 \\
\hline 5173 & 5177 & 1 & 0.1097 & 276646.08 \\
\hline 5173 & 5178 & 1 & 0.1097 & 276646.08 \\
\hline 5172 & 5179 & 1 & 0.1097 & 276646.08 \\
\hline 5172 & 5180 & 1 & 0.1097 & 276646.08 \\
\hline 5165 & 5194 & 1 & 0.1097 & 276646.08 \\
\hline 5160 & 5161 & 1 & 0.1097 & 276646.08 \\
\hline 5160 & 5162 & 1 & 0.1097 & 276646.08 \\
\hline 5160 & 5163 & 1 & 0.1097 & 276646.08 \\
\hline 5154 & 5196 & 1 & 0.1084 & 292126.88 \\
\hline 5148 & 5149 & 1 & 0.1097 & 276646.08 \\
\hline 5148 & 5150 & 1 & 0.1097 & 276646.08 \\
\hline 5148 & 5151 & 1 & 0.1097 & 276646.08 \\
\hline 5147 & 5152 & 1 & 0.1097 & 276646.08 \\
\hline 5147 & 5153 & 1 & 0.1097 & 276646.08 \\
\hline 5143 & 5144 & 1 & 0.1097 & 276646.08 \\
\hline 5143 & 5145 & 1 & 0.1097 & 276646.08 \\
\hline 5143 & 5146 & 1 & 0.1097 & 276646.08 \\
\hline 5137 & 5197 & 1 & 0.1084 & 292126.88 \\
\hline 5133 & 5134 & 1 & 0.1086 & 289365.44 \\
\hline 5133 & 5135 & 1 & 0.1086 & 289365.44 \\
\hline 5132 & 5136 & 1 & 0.1084 & 292126.88 \\
\hline 5128 & 5129 & 1 & 0.1097 & 276646.08 \\
\hline 5128 & 5130 & 1 & 0.1097 & 276646.08 \\
\hline 5128 & 5131 & 1 & 0.1097 & 276646.08 \\
\hline 5124 & 5199 & 1 & 0.2055 & 82266 \\
\hline 5139 & 5199 & 1 & 0.2055 & 82266 \\
\hline 5156 & 5199 & 1 & 0.2055 & 82266 \\
\hline 5168 & 5199 & 1 & 0.2055 & 82266 \\
\hline \multicolumn{5}{|l|}{ [ pairs ] } \\
\hline ai & aj & funct & & \\
\hline 5187 & 5195 & 1 & & \\
\hline 5181 & 5185 & 1 & & \\
\hline 5172 & 5181 & 1 & & \\
\hline 5172 & 5175 & 1 & & \\
\hline 5172 & 5176 & 1 & & \\
\hline 5171 & 5185 & 1 & & \\
\hline 5171 & 5174 & 1 & & \\
\hline 5170 & 5198 & 1 & & \\
\hline 5170 & 5173 & 1 & & \\
\hline 5169 & 5172 & 1 & & \\
\hline 5168 & 5172 & 1 & & \\
\hline 5168 & 5181 & 1 & & \\
\hline 5168 & 5198 & 1 & & \\
\hline 5167 & 5187 & 1 & & \\
\hline 5167 & 5185 & 1 & & \\
\hline 5167 & 5181 & 1 & & \\
\hline 5167 & 5173 & 1 & & \\
\hline 5166 & 5195 & 1 & & \\
\hline 5166 & 5188 & 1 & & \\
\hline 5166 & 5193 & 1 & & \\
\hline 5166 & 5169 & 1 & & \\
\hline 5166 & 5170 & 1 & & \\
\hline 5166 & 5172 & 1 & & \\
\hline 5165 & 5168 & 1 & & \\
\hline 5165 & 5171 & 1 & & \\
\hline 5165 & 5189 & 1 & & \\
\hline 5164 & 5167 & 1 & & \\
\hline 5164 & 5188 & 1 & & \\
\hline 5164 & 5193 & 1 & & \\
\hline
\end{tabular}




\begin{tabular}{|c|c|}
\hline 5160 & 5164 \\
\hline 5159 & 5166 \\
\hline 5159 & 5165 \\
\hline 5159 & 5195 \\
\hline 5158 & 5167 \\
\hline 5158 & 5187 \\
\hline 5157 & 5160 \\
\hline 5157 & 5195 \\
\hline 5157 & 5187 \\
\hline 5157 & 5168 \\
\hline 5157 & 5171 \\
\hline 5156 & 5160 \\
\hline 5156 & 5164 \\
\hline 5156 & 5165 \\
\hline 5156 & 5167 \\
\hline 5155 & 5166 \\
\hline 5155 & 5164 \\
\hline 5154 & 5157 \\
\hline 5154 & 5158 \\
\hline 5154 & 5160 \\
\hline 5147 & 5154 \\
\hline 5143 & 5147 \\
\hline 5142 & 5154 \\
\hline 5142 & 5148 \\
\hline 5141 & 5155 \\
\hline 5140 & 5143 \\
\hline 5140 & 5148 \\
\hline 5140 & 5156 \\
\hline 5140 & 5159 \\
\hline 5139 & 5143 \\
\hline 5139 & 5147 \\
\hline 5139 & 5155 \\
\hline 5138 & 5154 \\
\hline 5138 & 5147 \\
\hline 5137 & 5198 \\
\hline 5137 & 5140 \\
\hline 5137 & 5141 \\
\hline 5137 & 5143 \\
\hline 5132 & 5137 \\
\hline 5132 & 5198 \\
\hline 5128 & 5132 \\
\hline 5128 & 5185 \\
\hline 5127 & 5137 \\
\hline 5127 & 5133 \\
\hline 5127 & 5169 \\
\hline 5126 & 5138 \\
\hline 5126 & 5185 \\
\hline 5125 & 5185 \\
\hline 5125 & 5128 \\
\hline 5125 & 5133 \\
\hline 5125 & 5139 \\
\hline 5125 & 5142 \\
\hline 5124 & 5132 \\
\hline 5124 & 5138 \\
\hline 5124 & 5128 \\
\hline 5124 & 5169 \\
\hline 5194 & 5195 \\
\hline 5193 & 5194 \\
\hline 5188 & 5194 \\
\hline 5187 & 5190 \\
\hline 5187 & 5191 \\
\hline 5187 & 5192 \\
\hline 5178 & 5179 \\
\hline 5178 & 5180 \\
\hline 5177 & 5179 \\
\hline 5177 & 5180 \\
\hline 5176 & 5177 \\
\hline 5176 & 5178 \\
\hline 5175 & 5177 \\
\hline 5175 & 5178 \\
\hline 5174 & 5179 \\
\hline 5174 & 5180 \\
\hline 5171 & 5182 \\
\hline 5171 & 5183 \\
\hline 5171 & 5184 \\
\hline 5171 & 5177 \\
\hline 5171 & 5178 \\
\hline 5170 & 5186 \\
\hline 5170 & 5179 \\
\hline 5170 & 5180 \\
\hline 5169 & 5182 \\
\hline 5169 & 5183 \\
\hline 5169 & 5184 \\
\hline 5168 & 5186 \\
\hline 5167 & 5194 \\
\hline & 5179 \\
\hline
\end{tabular}




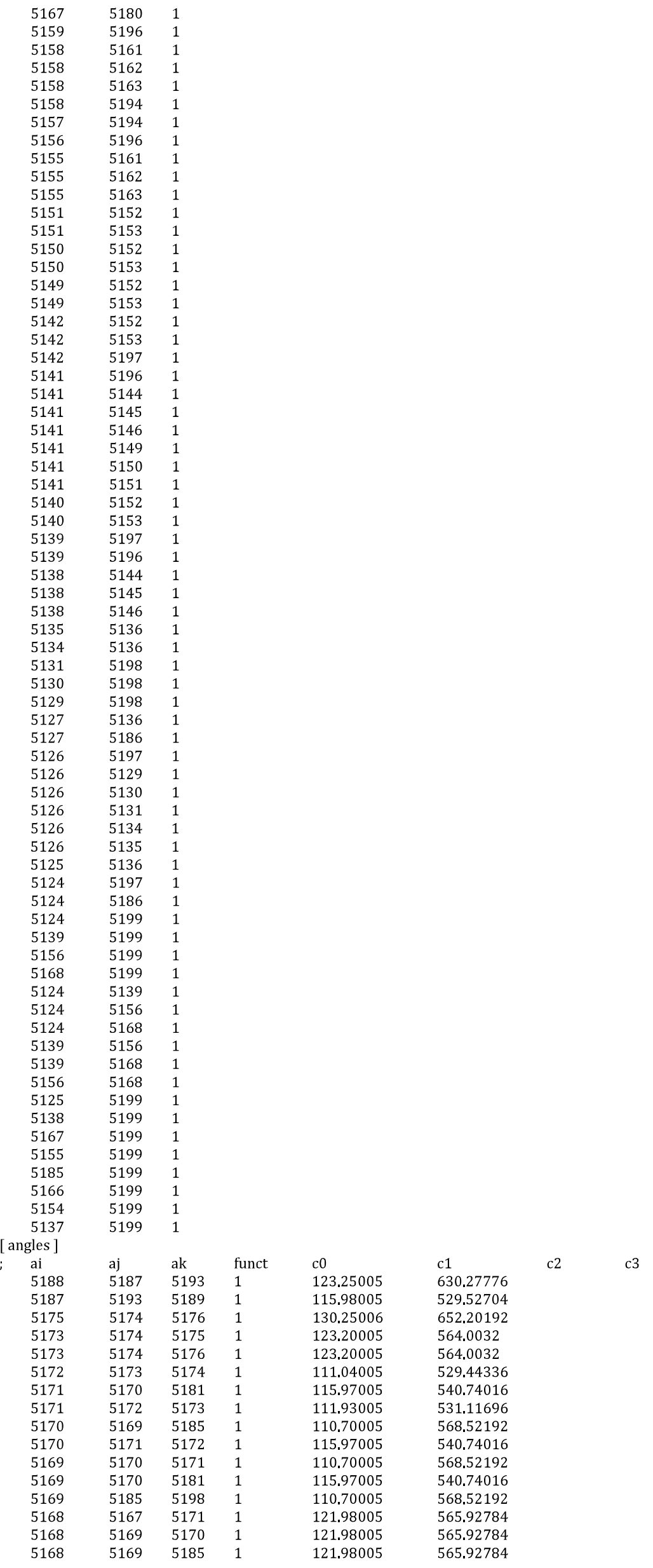




\begin{tabular}{|c|c|c|c|c|c|}
\hline 5167 & 5168 & 5169 & 1 & 103.76004 & 594.37904 \\
\hline 5167 & 5171 & 5170 & 1 & 110.70005 & 568.52192 \\
\hline 5167 & 5171 & 5172 & 1 & 115.97005 & 540.74016 \\
\hline 5166 & 5165 & 5187 & 1 & 113.17005 & 530.61488 \\
\hline 5166 & 5167 & 5168 & 1 & 121.98005 & 565.92784 \\
\hline 5166 & 5167 & 5171 & 1 & 110.70005 & 568.52192 \\
\hline 5165 & 5164 & 5195 & 1 & 123.20005 & 564.0032 \\
\hline 5165 & 5166 & 5167 & 1 & 115.97005 & 540.74016 \\
\hline 5165 & 5187 & 5188 & 1 & 110.72005 & 576.47152 \\
\hline 5165 & 5187 & 5193 & 1 & 123.20005 & 564.0032 \\
\hline 5164 & 5165 & 5166 & 1 & 113.17005 & 530.61488 \\
\hline 5164 & 5165 & 5187 & 1 & 111.63005 & 530.36384 \\
\hline 5159 & 5158 & 5164 & 1 & 122.69005 & 532.37216 \\
\hline 5158 & 5157 & 5166 & 1 & 110.70005 & 568.52192 \\
\hline 5158 & 5159 & 5160 & 1 & 115.97005 & 540.74016 \\
\hline 5158 & 5164 & 5165 & 1 & 117.29005 & 526.7656 \\
\hline 5158 & 5164 & 5195 & 1 & 123.93005 & 578.56352 \\
\hline 5157 & 5158 & 5159 & 1 & 110.70005 & 568.52192 \\
\hline 5157 & 5158 & 5164 & 1 & 122.69005 & 532.37216 \\
\hline 5157 & 5166 & 5165 & 1 & 115.97005 & 540.74016 \\
\hline 5157 & 5166 & 5167 & 1 & 110.70005 & 568.52192 \\
\hline 5156 & 5155 & 5159 & 1 & 121.98005 & 565.92784 \\
\hline 5156 & 5157 & 5158 & 1 & 121.98005 & 565.92784 \\
\hline 5156 & 5157 & 5166 & 1 & 121.98005 & 565.92784 \\
\hline 5155 & 5156 & 5157 & 1 & 103.76004 & 594.37904 \\
\hline 5155 & 5159 & 5158 & 1 & 110.70005 & 568.52192 \\
\hline 5155 & 5159 & 5160 & 1 & 115.97005 & 540.74016 \\
\hline 5154 & 5155 & 5156 & 1 & 121.98005 & 565.92784 \\
\hline 5154 & 5155 & 5159 & 1 & 110.70005 & 568.52192 \\
\hline 5142 & 5141 & 5147 & 1 & 115.97005 & 540.74016 \\
\hline 5141 & 5140 & 5154 & 1 & 110.70005 & 568.52192 \\
\hline 5141 & 5142 & 5143 & 1 & 115.97005 & 540.74016 \\
\hline 5141 & 5147 & 5148 & 1 & 111.93005 & 531.11696 \\
\hline 5140 & 5141 & 5142 & 1 & 110.70005 & 568.52192 \\
\hline 5140 & 5141 & 5147 & 1 & 115.97005 & 540.74016 \\
\hline 5140 & 5154 & 5155 & 1 & 110.70005 & 568.52192 \\
\hline 5139 & 5138 & 5142 & 1 & 121.98005 & 565.92784 \\
\hline 5139 & 5140 & 5141 & 1 & 121.98005 & 565.92784 \\
\hline 5139 & 5140 & 5154 & 1 & 121.98005 & 565.92784 \\
\hline 5138 & 5139 & 5140 & 1 & 103.76004 & 594.37904 \\
\hline 5138 & 5142 & 5141 & 1 & 110.70005 & 568.52192 \\
\hline 5138 & 5142 & 5143 & 1 & 115.97005 & 540.74016 \\
\hline 5137 & 5138 & 5139 & 1 & 121.98005 & 565.92784 \\
\hline 5137 & 5138 & 5142 & 1 & 110.70005 & 568.52192 \\
\hline 5128 & 5127 & 5198 & 1 & 115.97005 & 540.74016 \\
\hline 5127 & 5126 & 5132 & 1 & 110.70005 & 568.52192 \\
\hline 5127 & 5198 & 5185 & 1 & 110.70005 & 568.52192 \\
\hline 5126 & 5125 & 5137 & 1 & 110.70005 & 568.52192 \\
\hline 5126 & 5127 & 5128 & 1 & 115.97005 & 540.74016 \\
\hline 5126 & 5127 & 5198 & 1 & 110.70005 & 568.52192 \\
\hline 5126 & 5132 & 5133 & 1 & 111.04005 & 562.07856 \\
\hline 5125 & 5124 & 5198 & 1 & 103.76004 & 594.37904 \\
\hline 5125 & 5126 & 5127 & 1 & 110.70005 & 568.52192 \\
\hline 5125 & 5126 & 5132 & 1 & 110.70005 & 568.52192 \\
\hline 5125 & 5137 & 5138 & 1 & 110.70005 & 568.52192 \\
\hline 5124 & 5125 & 5126 & 1 & 121.98005 & 565.92784 \\
\hline 5124 & 5125 & 5137 & 1 & 121.98005 & 565.92784 \\
\hline 5124 & 5198 & 5127 & 1 & 121.98005 & 565.92784 \\
\hline 5124 & 5198 & 5185 & 1 & 121.98005 & 565.92784 \\
\hline 5191 & 5189 & 5192 & 1 & 108.46005 & 328.36032 \\
\hline 5190 & 5189 & 5191 & 1 & 108.46005 & 328.36032 \\
\hline 5190 & 5189 & 5192 & 1 & 108.46005 & 328.36032 \\
\hline 5188 & 5189 & 5190 & 1 & 109.78005 & 425.0944 \\
\hline 5193 & 5189 & 5191 & 1 & 109.78005 & 425.0944 \\
\hline 5193 & 5189 & 5192 & 1 & 109.78005 & 425.0944 \\
\hline 5187 & 5165 & 5194 & 1 & 108.77005 & 392.71024 \\
\hline 5186 & 5185 & 5198 & 1 & 121.07005 & 394.5512 \\
\hline 5183 & 5181 & 5184 & 1 & 107.58005 & 329.6992 \\
\hline 5182 & 5181 & 5183 & 1 & 107.58005 & 329.6992 \\
\hline 5182 & 5181 & 5184 & 1 & 107.58005 & 329.6992 \\
\hline 5179 & 5172 & 5180 & 1 & 107.58005 & 329.6992 \\
\hline 5177 & 5173 & 5178 & 1 & 107.58005 & 329.6992 \\
\hline 5174 & 5173 & 5177 & 1 & 108.77005 & 392.71024 \\
\hline 5174 & 5173 & 5178 & 1 & 108.77005 & 392.71024 \\
\hline 5173 & 5172 & 5179 & 1 & 109.80005 & 387.77312 \\
\hline 5173 & 5172 & 5180 & 1 & 109.80005 & 387.77312 \\
\hline 5172 & 5173 & 5177 & 1 & 109.80005 & 387.77312 \\
\hline 5172 & 5173 & 5178 & 1 & 109.80005 & 387.77312 \\
\hline 5171 & 5172 & 5179 & 1 & 110.49005 & 394.80224 \\
\hline 5171 & 5172 & 5180 & 1 & 110.49005 & 394.80224 \\
\hline 5170 & 5181 & 5182 & 1 & 110.49005 & 394.80224 \\
\hline 5170 & 5181 & 5183 & 1 & 110.49005 & 394.80224 \\
\hline 5170 & 5181 & 5184 & 1 & 110.49005 & 394.80224 \\
\hline 5169 & 5185 & 5186 & 1 & 121.07005 & 394.5512 \\
\hline 5166 & 5165 & 5194 & 1 & 110.49005 & 394.80224 \\
\hline 5164 & 5165 & 5194 & 1 & 108.77005 & 392.71024 \\
\hline
\end{tabular}




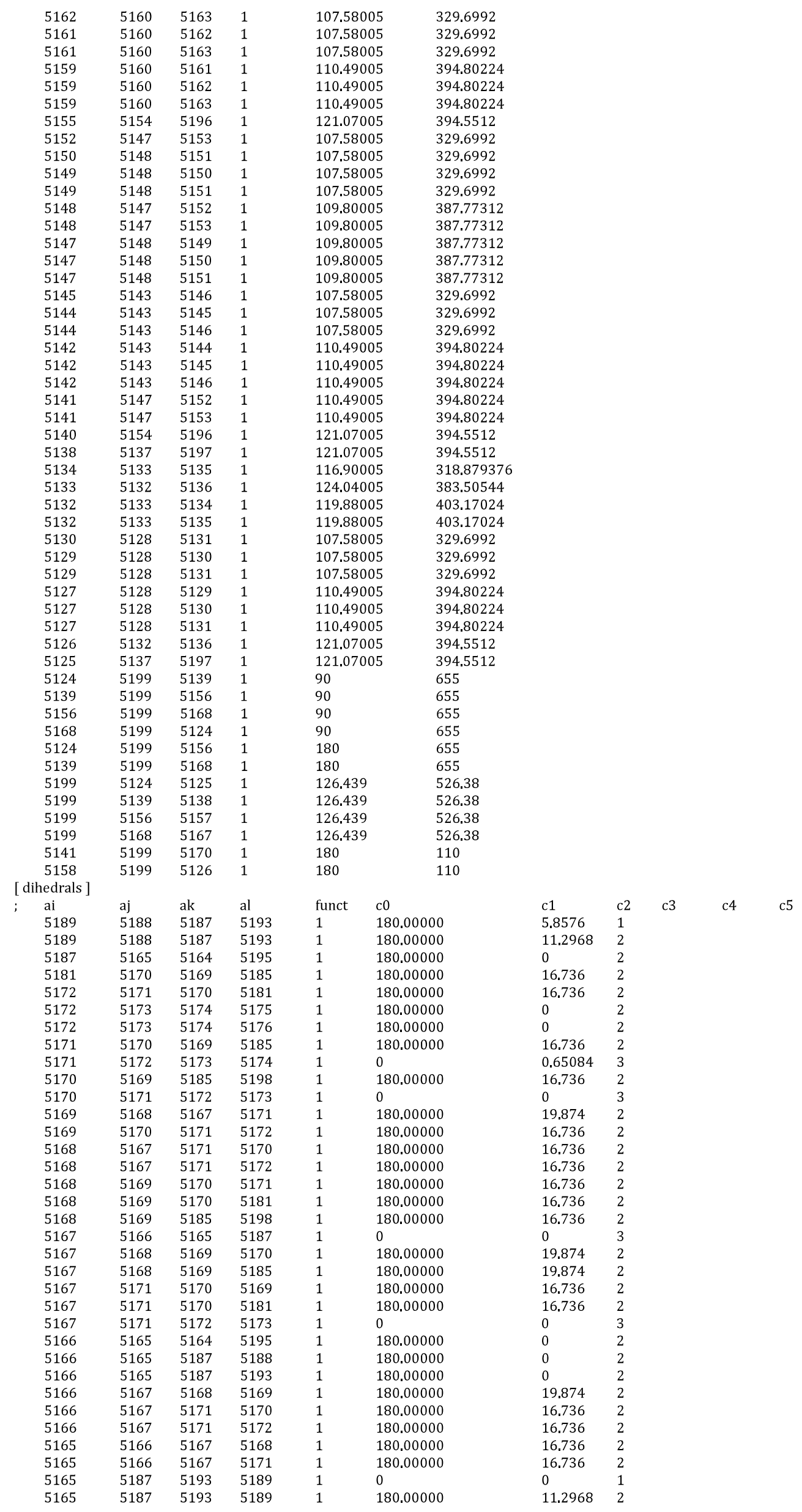




\begin{tabular}{|c|c|c|c|c|c|c|}
\hline 5165 & 5187 & 5193 & 5189 & 1 & 0 & 4.8116 \\
\hline 5164 & 5158 & 5157 & 5166 & 1 & 180.00000 & 16.736 \\
\hline 5164 & 5165 & 5166 & 5167 & 1 & 0 & 0 \\
\hline 5164 & 5165 & 5187 & 5188 & 1 & 180.00000 & 0 \\
\hline 5164 & 5165 & 5187 & 5193 & 1 & 180.00000 & 0 \\
\hline 5160 & 5159 & 5158 & 5164 & 1 & 180.00000 & 16.736 \\
\hline 5159 & 5158 & 5157 & 5166 & 1 & 180.00000 & 16.736 \\
\hline 5159 & 5158 & 5164 & 5165 & 1 & 180.00000 & 12.029 \\
\hline 5159 & 5158 & 5164 & 5195 & 1 & 180.00000 & 12.029 \\
\hline 5158 & 5157 & 5166 & 5165 & 1 & 180.00000 & 16.736 \\
\hline 5158 & 5157 & 5166 & 5167 & 1 & 180.00000 & 16.736 \\
\hline 5158 & 5164 & 5165 & 5166 & 1 & 180.00000 & 0 \\
\hline 5158 & 5164 & 5165 & 5187 & 1 & 180.00000 & 0 \\
\hline 5157 & 5156 & 5155 & 5159 & 1 & 180.00000 & 19.874 \\
\hline 5157 & 5158 & 5159 & 5160 & 1 & 180.00000 & 16.736 \\
\hline 5157 & 5158 & 5164 & 5165 & 1 & 180.00000 & 12.029 \\
\hline 5157 & 5158 & 5164 & 5195 & 1 & 180.00000 & 12.029 \\
\hline 5157 & 5166 & 5165 & 5164 & 1 & 0 & 0 \\
\hline 5157 & 5166 & 5165 & 5187 & 1 & 0 & 0 \\
\hline 5157 & 5166 & 5167 & 5168 & 1 & 180.00000 & 16.736 \\
\hline 5157 & 5166 & 5167 & 5171 & 1 & 180.00000 & 16.736 \\
\hline 5156 & 5155 & 5159 & 5158 & 1 & 180.00000 & 16.736 \\
\hline 5156 & 5155 & 5159 & 5160 & 1 & 180.00000 & 16.736 \\
\hline 5156 & 5157 & 5158 & 5159 & 1 & 180.00000 & 16.736 \\
\hline 5156 & 5157 & 5158 & 5164 & 1 & 180.00000 & 16.736 \\
\hline 5156 & 5157 & 5166 & 5165 & 1 & 180.00000 & 16.736 \\
\hline 5156 & 5157 & 5166 & 5167 & 1 & 180.00000 & 16.736 \\
\hline 5155 & 5156 & 5157 & 5158 & 1 & 180.00000 & 19.874 \\
\hline 5155 & 5156 & 5157 & 5166 & 1 & 180.00000 & 19.874 \\
\hline 5155 & 5159 & 5158 & 5157 & 1 & 180.00000 & 16.736 \\
\hline 5155 & 5159 & 5158 & 5164 & 1 & 180.00000 & 16.736 \\
\hline 5154 & 5155 & 5156 & 5157 & 1 & 180.00000 & 19.874 \\
\hline 5154 & 5155 & 5159 & 5158 & 1 & 180.00000 & 16.736 \\
\hline 5154 & 5155 & 5159 & 5160 & 1 & 180.00000 & 16.736 \\
\hline 5147 & 5141 & 5140 & 5154 & 1 & 180.00000 & 16.736 \\
\hline 5143 & 5142 & 5141 & 5147 & 1 & 180.00000 & 16.736 \\
\hline 5142 & 5141 & 5140 & 5154 & 1 & 180.00000 & 16.736 \\
\hline 5142 & 5141 & 5147 & 5148 & 1 & 0 & 0 \\
\hline 5141 & 5140 & 5154 & 5155 & 1 & 180.00000 & 16.736 \\
\hline 5140 & 5139 & 5138 & 5142 & 1 & 180.00000 & 19.874 \\
\hline 5140 & 5141 & 5142 & 5143 & 1 & 180.00000 & 16.736 \\
\hline 5140 & 5141 & 5147 & 5148 & 1 & 0 & 0 \\
\hline 5140 & 5154 & 5155 & 5156 & 1 & 180.00000 & 16.736 \\
\hline 5140 & 5154 & 5155 & 5159 & 1 & 180.00000 & 16.736 \\
\hline 5139 & 5138 & 5142 & 5141 & 1 & 180.00000 & 16.736 \\
\hline 5139 & 5138 & 5142 & 5143 & 1 & 180.00000 & 16.736 \\
\hline 5139 & 5140 & 5141 & 5142 & 1 & 180.00000 & 16.736 \\
\hline 5139 & 5140 & 5141 & 5147 & 1 & 180.00000 & 16.736 \\
\hline 5139 & 5140 & 5154 & 5155 & 1 & 180.00000 & 16.736 \\
\hline 5138 & 5139 & 5140 & 5141 & 1 & 180.00000 & 19.874 \\
\hline 5138 & 5139 & 5140 & 5154 & 1 & 180.00000 & 19.874 \\
\hline 5138 & 5142 & 5141 & 5140 & 1 & 180.00000 & 16.736 \\
\hline 5138 & 5142 & 5141 & 5147 & 1 & 180.00000 & 16.736 \\
\hline 5137 & 5125 & 5124 & 5198 & 1 & 180.00000 & 19.874 \\
\hline 5137 & 5138 & 5139 & 5140 & 1 & 180.00000 & 19.874 \\
\hline 5137 & 5138 & 5142 & 5141 & 1 & 180.00000 & 16.736 \\
\hline 5137 & 5138 & 5142 & 5143 & 1 & 180.00000 & 16.736 \\
\hline 5132 & 5126 & 5125 & 5137 & 1 & 180.00000 & 16.736 \\
\hline 5132 & 5126 & 5127 & 5198 & 1 & 180.00000 & 16.736 \\
\hline 5128 & 5127 & 5126 & 5132 & 1 & 180.00000 & 16.736 \\
\hline 5128 & 5127 & 5198 & 5185 & 1 & 180,00000 & 16.736 \\
\hline 5127 & 5126 & 5125 & 5137 & 1 & 180.00000 & 16.736 \\
\hline 5127 & 5126 & 5132 & 5133 & 1 & 180.00000 & 16.736 \\
\hline 5127 & 5198 & 5185 & 5169 & 1 & 180.00000 & 16.736 \\
\hline 5126 & 5125 & 5124 & 5198 & 1 & 180.00000 & 19.874 \\
\hline 5126 & 5125 & 5137 & 5138 & 1 & 180.00000 & 16.736 \\
\hline 5126 & 5127 & 5198 & 5185 & 1 & 180.00000 & 16.736 \\
\hline 5125 & 5124 & 5198 & 5127 & 1 & 180.00000 & 19.874 \\
\hline 5125 & 5124 & 5198 & 5185 & 1 & 180.00000 & 19.874 \\
\hline 5125 & 5126 & 5127 & 5128 & 1 & 180.00000 & 16.736 \\
\hline 5125 & 5126 & 5127 & 5198 & 1 & 180.00000 & 16.736 \\
\hline 5125 & 5126 & 5132 & 5133 & 1 & 180.00000 & 16.736 \\
\hline 5125 & 5137 & 5138 & 5139 & 1 & 180.00000 & 16.736 \\
\hline 5125 & 5137 & 5138 & 5142 & 1 & 180.00000 & 16.736 \\
\hline 5124 & 5125 & 5126 & 5127 & 1 & 180.00000 & 16.736 \\
\hline 5124 & 5125 & 5126 & 5132 & 1 & 180.00000 & 16.736 \\
\hline 5124 & 5125 & 5137 & 5138 & 1 & 180.00000 & 16.736 \\
\hline 5124 & 5198 & 5127 & 5126 & 1 & 180.00000 & 16.736 \\
\hline 5124 & 5198 & 5127 & 5128 & 1 & 180.00000 & 16.736 \\
\hline 5124 & 5198 & 5185 & 5169 & 1 & 180.00000 & 16.736 \\
\hline 5127 & 5185 & 5198 & 5124 & 4 & 180,00000 & 4.6024 \\
\hline 5165 & 5193 & 5187 & 5188 & 4 & 180.00000 & 4.6024 \\
\hline 5173 & 5175 & 5174 & 5176 & 4 & 180.00000 & 4.6024 \\
\hline 5170 & 5167 & 5171 & 5172 & 4 & 180.00000 & 4.6024 \\
\hline 5171 & 5169 & 5170 & 5181 & 4 & 180.00000 & 4.6024 \\
\hline 5170 & 5185 & 5169 & 5168 & 4 & 180.00000 & 4.6024 \\
\hline
\end{tabular}




\begin{tabular}{|c|c|c|c|c|c|c|}
\hline 5171 & 5166 & 5167 & 5168 & 4 & 180.00000 & 4.6024 \\
\hline 5157 & 5167 & 5166 & 5165 & 4 & 180.00000 & 4.6024 \\
\hline 5158 & 5165 & 5164 & 5195 & 4 & 180.00000 & 43.932 \\
\hline 5158 & 5155 & 5159 & 5160 & 4 & 180.00000 & 4.6024 \\
\hline 5164 & 5159 & 5158 & 5157 & 4 & 180.00000 & 4.6024 \\
\hline 5158 & 5166 & 5157 & 5156 & 4 & 180.00000 & 4.6024 \\
\hline 5159 & 5154 & 5155 & 5156 & 4 & 180.00000 & 4.6024 \\
\hline 5141 & 5138 & 5142 & 5143 & 4 & 180.00000 & 4.6024 \\
\hline 5142 & 5140 & 5141 & 5147 & 4 & 180.00000 & 4.6024 \\
\hline 5141 & 5154 & 5140 & 5139 & 4 & 180.00000 & 4.6024 \\
\hline 5142 & 5137 & 5138 & 5139 & 4 & 180.00000 & 4.6024 \\
\hline 5126 & 5198 & 5127 & 5128 & 4 & 180.00000 & 4.6024 \\
\hline 5127 & 5125 & 5126 & 5132 & 4 & 180.00000 & 4.6024 \\
\hline 5126 & 5137 & 5125 & 5124 & 4 & 180.00000 & 4.6024 \\
\hline 5194 & 5165 & 5164 & 5195 & 1 & 0 & 3.3472 \\
\hline 5194 & 5165 & 5164 & 5195 & 1 & 0 & 0 \\
\hline 5194 & 5165 & 5164 & 5195 & 1 & 180.00000 & 0.33472 \\
\hline 5193 & 5187 & 5165 & 5194 & 1 & 0 & 3.3472 \\
\hline 5193 & 5187 & 5165 & 5194 & 1 & 0 & 0 \\
\hline 5193 & 5187 & 5165 & 5194 & 1 & 180.00000 & 0.33472 \\
\hline 5188 & 5187 & 5165 & 5194 & 1 & 180.00000 & 0 \\
\hline 5187 & 5193 & 5189 & 5190 & 1 & 0 & 1.60387 \\
\hline 5187 & 5193 & 5189 & 5191 & 1 & 0 & 1.60387 \\
\hline 5187 & 5193 & 5189 & 5192 & 1 & 0 & 1.60387 \\
\hline 5178 & 5173 & 5172 & 5179 & 1 & 0 & 0.6276 \\
\hline 5178 & 5173 & 5172 & 5180 & 1 & 0 & 0.6276 \\
\hline 5177 & 5173 & 5172 & 5179 & 1 & 0 & 0.6276 \\
\hline 5177 & 5173 & 5172 & 5180 & 1 & 0 & 0.6276 \\
\hline 5176 & 5174 & 5173 & 5177 & 1 & 0 & 3.3472 \\
\hline 5176 & 5174 & 5173 & 5177 & 1 & 0 & 0 \\
\hline 5176 & 5174 & 5173 & 5177 & 1 & 180.00000 & 0.33472 \\
\hline 5176 & 5174 & 5173 & 5178 & 1 & 0 & 3.3472 \\
\hline 5176 & 5174 & 5173 & 5178 & 1 & 0 & 0 \\
\hline 5176 & 5174 & 5173 & 5178 & 1 & 180.00000 & 0.33472 \\
\hline 5175 & 5174 & 5173 & 5177 & 1 & 0 & 3.3472 \\
\hline 5175 & 5174 & 5173 & 5177 & 1 & 0 & 0 \\
\hline 5175 & 5174 & 5173 & 5177 & 1 & 180.00000 & 0.33472 \\
\hline 5175 & 5174 & 5173 & 5178 & 1 & 0 & 3.3472 \\
\hline 5175 & 5174 & 5173 & 5178 & 1 & 0 & 0 \\
\hline 5175 & 5174 & 5173 & 5178 & 1 & 180.00000 & 0.33472 \\
\hline 5174 & 5173 & 5172 & 5179 & 1 & 0 & 0.65084 \\
\hline 5174 & 5173 & 5172 & 5180 & 1 & 0 & 0.65084 \\
\hline 5171 & 5170 & 5181 & 5182 & 1 & 0 & 0 \\
\hline 5171 & 5170 & 5181 & 5183 & 1 & 0 & 0 \\
\hline 5171 & 5170 & 5181 & 5184 & 1 & 0 & 0 \\
\hline 5171 & 5172 & 5173 & 5177 & 1 & 0 & 0.65084 \\
\hline 5171 & 5172 & 5173 & 5178 & 1 & 0 & 0.65084 \\
\hline 5170 & 5169 & 5185 & 5186 & 1 & 180.00000 & 16.736 \\
\hline 5170 & 5171 & 5172 & 5179 & 1 & 0 & 0 \\
\hline 5170 & 5171 & 5172 & 5180 & 1 & 0 & 0 \\
\hline 5169 & 5170 & 5181 & 5182 & 1 & 0 & 0 \\
\hline 5169 & 5170 & 5181 & 5183 & 1 & 0 & 0 \\
\hline 5169 & 5170 & 5181 & 5184 & 1 & 0 & 0 \\
\hline 5168 & 5169 & 5185 & 5186 & 1 & 180.00000 & 16.736 \\
\hline 5167 & 5166 & 5165 & 5194 & 1 & 0 & 0 \\
\hline 5167 & 5171 & 5172 & 5179 & 1 & 0 & 0 \\
\hline 5167 & 5171 & 5172 & 5180 & 1 & 0 & 0 \\
\hline 5159 & 5155 & 5154 & 5196 & 1 & 180.00000 & 16.736 \\
\hline 5158 & 5159 & 5160 & 5161 & 1 & 0 & 0 \\
\hline 5158 & 5159 & 5160 & 5162 & 1 & 0 & 0 \\
\hline 5158 & 5159 & 5160 & 5163 & 1 & 0 & 0 \\
\hline 5158 & 5164 & 5165 & 5194 & 1 & 180.00000 & 0 \\
\hline 5157 & 5166 & 5165 & 5194 & 1 & 0 & 0 \\
\hline 5156 & 5155 & 5154 & 5196 & 1 & 180.00000 & 16.736 \\
\hline 5155 & 5159 & 5160 & 5161 & 1 & 0 & 0 \\
\hline 5155 & 5159 & 5160 & 5162 & 1 & 0 & 0 \\
\hline 5155 & 5159 & 5160 & 5163 & 1 & 0 & 0 \\
\hline 5151 & 5148 & 5147 & 5152 & 1 & 0 & 0.6276 \\
\hline 5151 & 5148 & 5147 & 5153 & 1 & 0 & 0.6276 \\
\hline 5150 & 5148 & 5147 & 5152 & 1 & 0 & 0.6276 \\
\hline 5150 & 5148 & 5147 & 5153 & 1 & 0 & 0.6276 \\
\hline 5149 & 5148 & 5147 & 5152 & 1 & 0 & 0.6276 \\
\hline 5149 & 5148 & 5147 & 5153 & 1 & 0 & 0.6276 \\
\hline 5142 & 5141 & 5147 & 5152 & 1 & 0 & 0 \\
\hline 5142 & 5141 & 5147 & 5153 & 1 & 0 & 0 \\
\hline 5142 & 5138 & 5137 & 5197 & 1 & 180.00000 & 16.736 \\
\hline 5141 & 5140 & 5154 & 5196 & 1 & 180,00000 & 16.736 \\
\hline 5141 & 5142 & 5143 & 5144 & 1 & 0 & 0 \\
\hline 5141 & 5142 & 5143 & 5145 & 1 & 0 & 0 \\
\hline 5141 & 5142 & 5143 & 5146 & 1 & 0 & 0 \\
\hline 5141 & 5147 & 5148 & 5149 & 1 & 0 & 0.65084 \\
\hline 5141 & 5147 & 5148 & 5150 & 1 & 0 & 0.65084 \\
\hline 5141 & 5147 & 5148 & 5151 & 1 & 0 & 0.65084 \\
\hline 5140 & 5141 & 5147 & 5152 & 1 & 0 & 0 \\
\hline 5140 & 5141 & 5147 & 5153 & 1 & 0 & 0 \\
\hline 5139 & 5138 & 5137 & 5197 & 1 & 180.00000 & 16.736 \\
\hline
\end{tabular}




\begin{tabular}{|c|c|c|c|c|c|c|}
\hline 5139 & 5140 & 5154 & 5196 & 1 & 180.00000 & 16.736 \\
\hline 5138 & 5142 & 5143 & 5144 & 1 & 0 & 0 \\
\hline 5138 & 5142 & 5143 & 5145 & 1 & 0 & 0 \\
\hline 5138 & 5142 & 5143 & 5146 & 1 & 0 & 0 \\
\hline 5135 & 5133 & 5132 & 5136 & 1 & 180.00000 & 2.9288 \\
\hline 5134 & 5133 & 5132 & 5136 & 1 & 180.00000 & 2.9288 \\
\hline 5131 & 5128 & 5127 & 5198 & 1 & 0 & 0 \\
\hline 5130 & 5128 & 5127 & 5198 & 1 & 0 & 0 \\
\hline 5129 & 5128 & 5127 & 5198 & 1 & 0 & 0 \\
\hline 5127 & 5126 & 5132 & 5136 & 1 & 180.00000 & 16.736 \\
\hline 5127 & 5198 & 5185 & 5186 & 1 & 180.00000 & 16.736 \\
\hline 5126 & 5125 & 5137 & 5197 & 1 & 180.00000 & 16.736 \\
\hline 5126 & 5127 & 5128 & 5129 & 1 & 0 & 0 \\
\hline 5126 & 5127 & 5128 & 5130 & 1 & 0 & 0 \\
\hline 5126 & 5127 & 5128 & 5131 & 1 & 0 & 0 \\
\hline 5126 & 5132 & 5133 & 5134 & 1 & 180.00000 & 2.9288 \\
\hline 5126 & 5132 & 5133 & 5135 & 1 & 180.00000 & 2.9288 \\
\hline 5125 & 5126 & 5132 & 5136 & 1 & 180.00000 & 16.736 \\
\hline 5124 & 5125 & 5137 & 5197 & 1 & 180.00000 & 16.736 \\
\hline 5124 & 5198 & 5185 & 5186 & 1 & 180.00000 & 16.736 \\
\hline 5169 & 5198 & 5185 & 5186 & 1 & 180.00000 & 4.6024 \\
\hline 5140 & 5155 & 5154 & 5196 & 1 & 180.00000 & 4.6024 \\
\hline 5125 & 5138 & 5137 & 5197 & 1 & 180.00000 & 4.6024 \\
\hline 5132 & 5134 & 5133 & 5135 & 1 & 180.00000 & 4.6024 \\
\hline 5126 & 5133 & 5132 & 5136 & 1 & 180.00000 & 4.6024 \\
\hline 5185 & 5198 & 5124 & 5199 & 4 & 0 & 49.712 \\
\hline 5166 & 5167 & 5168 & 5199 & 4 & 0 & 49.712 \\
\hline 5154 & 5155 & 5156 & 5199 & 4 & 0 & 49.712 \\
\hline 5137 & 5138 & 5139 & 5199 & 4 & 0 & 49.712 \\
\hline 5199 & 5124 & 5139 & 5156 & 4 & 0 & 49.712 \\
\hline 5125 & 5124 & 5198 & 5199 & 4 & -180 & 47.674 \\
\hline 5138 & 5139 & 5140 & 5199 & 4 & -180 & 47.674 \\
\hline 5155 & 5156 & 5157 & 5199 & 4 & -180 & 47.674 \\
\hline 5167 & 5168 & 5169 & 5199 & 4 & -180 & 47.674 \\
\hline
\end{tabular}

